\title{
Identification and analysis of novel insect head patterning genes
}

\author{
Dissertation \\ for the award of the degree \\ "Doctor rerum naturalium" \\ of the Georg-August-Universität Göttingen \\ submitted by \\ Janna Siemanowski \\ from Moers, Germany
}

Göttingen 2015 


\section{Thesis Committee}

Prof. Dr. Gregor Bucher (1st reviewer, advisor)

Johann-Friedrich-Blumenbach-Institute of Zoology and Anthropology, Department of Evolutionary Developmental Genetics, Georg-August-University Göttingen

Dr. Roland Dosch (2nd reviewer)

Department of Developmental Biochemistry, University Medical Center Göttingen

\section{Prof. Dr. Andreas Wodarz}

Department of Microscopic Anatomy and Molecular Cell Biology, Stem Cell Biology, University of Cologne

Date of oral examination: 


\section{Declaration}

Herewith I declare, that I prepared the Dissertation

„Identification and analysis of novel insect head patterning genes“

on my own and with no other sources and aids than quoted.

Janna Siemanowski

Göttingen, 25.03.2015 
Meinen Eltern

Edelgard Friedrich-Siemanowski und Werner Siemanowski 


\section{Danksagung}

An erster Stelle möchte ich mich außerordentlich bei Herrn Prof. Dr. Gregor Bucher für die Betreuung der Doktorarbeit bedanken! Ich danke ihm für die stets offene Tür, seinen wissenschaftlichen Hilfestellungen und hilfreichen Diskussionen, die diese Arbeit stets vorangebracht und fokussiert haben. Ein besondere Dank auch für die Bereitstellung eines Arbeitsplatzes innerhalb einer wunderbaren Arbeitsatmosphäre und aufmunternde Worte wenn sie von Nöten waren. Außerdem bedanke ich mich für die Möglichkeit an zahlreichen Tagungen teilzunehmen.

Vielen Dank an die Mitglieder meines Thesis Committee, Herrn Prof. Dr. Andreas Wodarz und Herrn Dr. Roland Dosch, für die freundliche und fachlich unterstützende Hilfestellung während der Meetings.

Mein besonderer Dank geht an Claudia Hinners für Ihre umfangreiche und tatkräftige Unterstützung in der täglichen Laborarbeit und speziell in diesem Projekt. Ohne Sie wäre der Arbeitsfluss im Labor nur halb so effektiv. Darüber hinaus möchte ich mich bei Ihr für all die netten Gespräche und aufmunternden Worte in jeglicher Form bedanken.

Herzlich Bedanken möchte ich mich auch bei Dr. Georg Oberhofer für etliche Diskussionen und Hilfestellungen bei der täglichen Laborarbeit. Ein besonderer Dank an ihn für die kritische Durchsicht dieser Arbeit. Darüber hinaus danke ich ihm für die tolle Unterstützung und jegliche Ablenkung vom Allstagsstress. Vielen Dank das du immer für mich da warst.

Ich bedanke mich bei Prof. Dr. Ernst Wimmer und Dr. Nico Posnien für umfangreiche und hilfreiche Diskussionen während den Abteilungsseminaren.

Ein ganz besonderer Danke gilt hier Nico Posnien für die Hilfestellung bei der statistischen Analyse meiner Daten.

Mein Dank geht außerdem an die Screener und dem ganzen Team rund um iBeetle, ohne die dieses Projekt überhaupt nicht möglich gewesen wäre. Speziell bedanken möchte ich mich hier bei Jonas Schwirz für die geduldige Einweisung in die Käfer und rescreen Arbeit.

Danke auch an meine Studenten Tobias Vollmer und Tatian Königsmann. 
Ein besonderer Dank geht natürlich an das gesamte Labor 3, inklusive jener, die kürzlich hinzu gekommen sind. Alle zusammen haben es geschafft einen lebhaft und lustigen Arbeitsalltag zu schaffen. Besonders danken möchte ich hier Julia Ulrich, Peter Kitzmann, Daniela Grossmann, Magdalena Schacht, Yonggang Hu und Elke Küsters.

Ebenso möchte ich mich recht herzlich bei Inga Schild, Birgit Rossi und Constanze Gerhards für all die freundliche und tatkräftige Hilfestellung bei der Bewältigung der bürokratischen Angelegenheiten bedanken.

Außerdem danke ich der gesamten Abteilung Entwicklungsbiologie für die wunderbare Arbeitsatmosphäre und Hilfsbereitschaft, die das Anfertigen dieser Arbeit in einem netten Umfeld umso erleichtert haben. Hervorheben möchte ich hier Sabrina Lehmann, Alice Montino und Bernhard Schmidt. Ich danke Ihnen für viele fruchtvolle Diskussionen während der Arbeit. Bedanken möchte ich mich jedoch auch für all die zahlreichen Gespräche und taten fernab jeglicher Ernsthaftigkeit, die das Arbeiten zu einer schönen Erfahrung gemacht haben und für die ständige Unterstützung wenn sie von Nöten war.

Zu guter Letzt möchte ich mich besonders bei meinen Eltern, meiner Schwester und meinen Freunden für die ständige Unterstützung in jeglicher Form in den letzten Jahren Bedanken. Euer ständiger Rückhalt hat alles erleichtert! 


\section{Table of Contents}

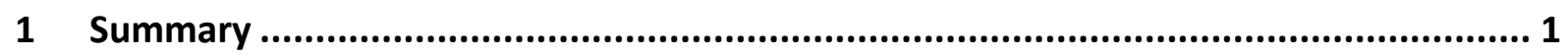

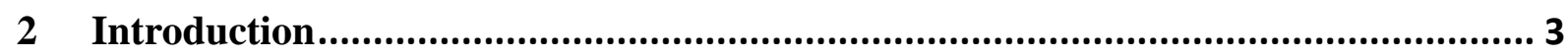

2.1 Segmental composition of the insect head .......................................................... 3

2.2 Head patterning in insects............................................................................ 4

2.3 Using Tribolium castaneum as a model for insect head patterning........................... 6

2.4 Direct comparison of Drosphila and Tribolium head patterning ................................ 7

2.5 The labrum arises in the anterior median region (AMR) ………………………..... 7

2.6 Identification of novel head patterning genes....................................................... 9

2.7 The developmental function of Notch signaling ................................................ 9

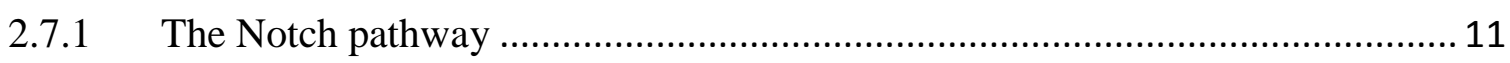

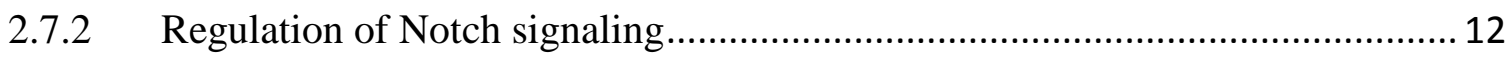

2.7.3 The Notch signaling pathway in Tribolium................................................... 14

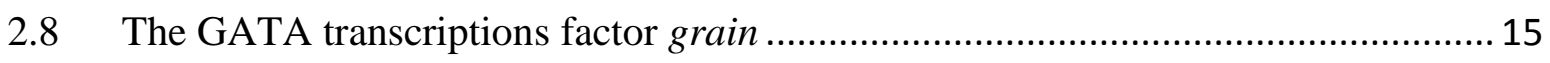

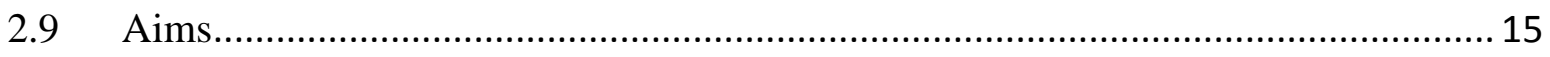

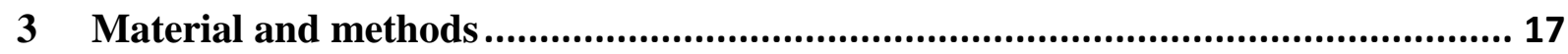

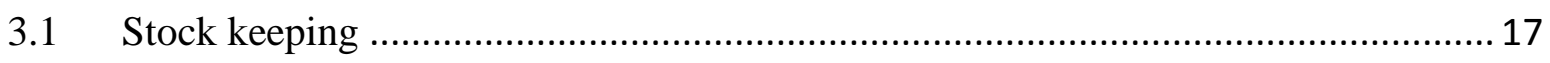

3.2 Candidate gene selection ............................................................................. 17

3.3 Orthology and phylogenetic analysis ........................................................... 17

$3.4 \quad$ Molecular biology ......................................................................................... 18

3.5 Knock down by RNA interference (RNAi) and injection techniques ....................... 19

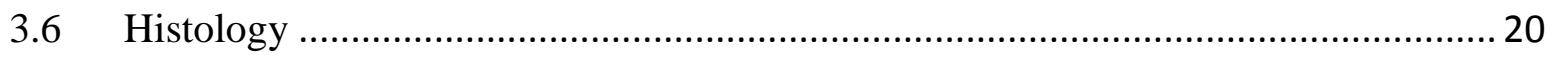

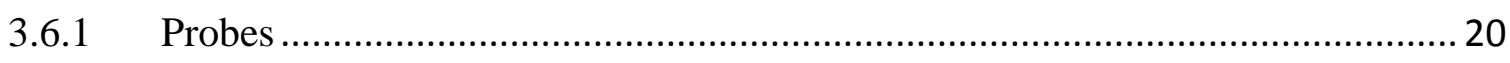

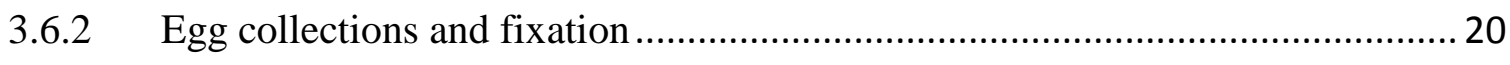

3.6.3 Whole mount in situ hybridization (ISH) ……………................................... 20

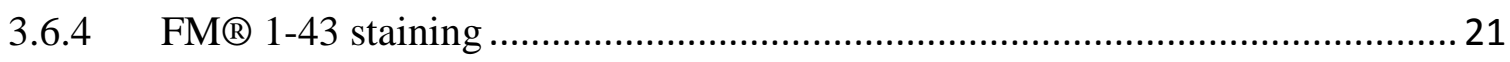

3.6.5 Cleaved Drosophila death caspase-1 (Dcp-1) staining .................................... 21

3.6.6 Edu proliferation assay and analysis .............................................................. 21

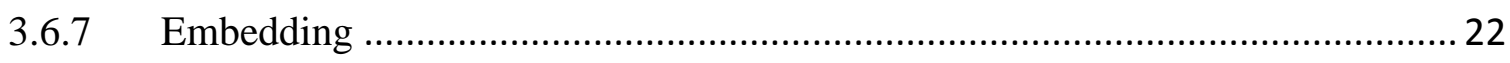

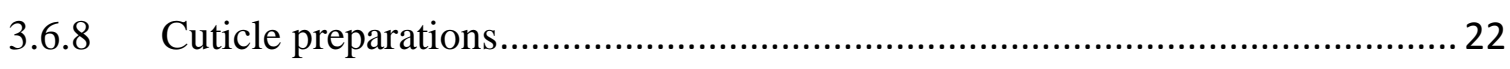

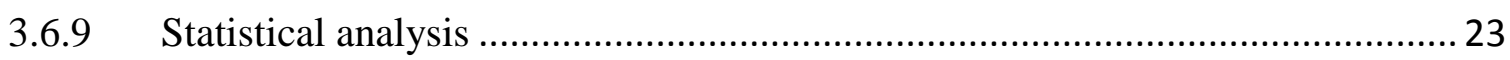




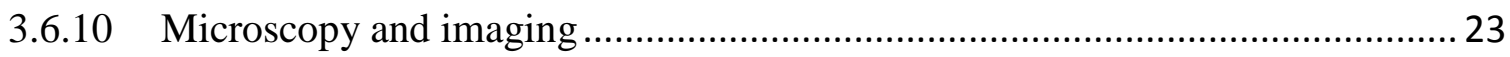

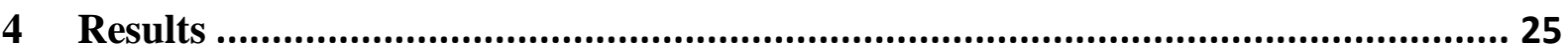

4.1 Selection of head phenotypes from the iBeetle screen .......................................... 25

4.1.1 Rescreen and selection of final candidate genes .......................................... 26

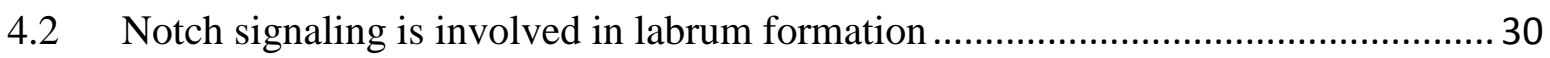

4.2.1 iB_05634 (Tc-mind bomb 1) and iB_04764 (Tc-Serrate) identified as genes both

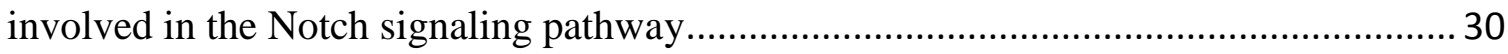

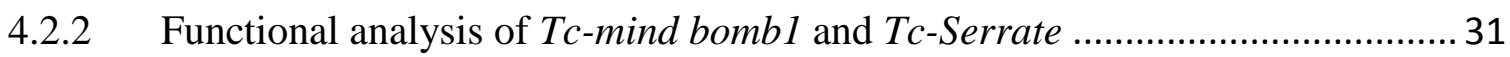

4.2.3 Labrum development is initialized after disruption of Notch signaling pathway

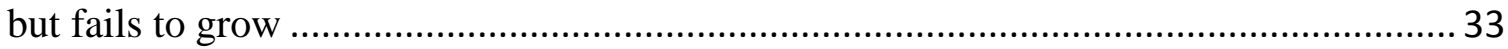

4.2.4 Notch signaling components are active in developing labrum and leg anlagen 35

4.2.5 Notch signaling is not acting upstream of the AMR network ........................... 36

4.2.6 Role of Notch signaling in the appendage regulatory gene network ................. 40

4.2.7 Expression dynamics of proximal-distal patterning genes in labrum and legs .. 40

4.2.8 Changes in expression after Tc-mib1 RNAi ................................................. 44

4.2.9 Notch signaling regulates cell proliferation during early labrum formation ...... 49

4.2.10 Tissue loss of labrum after knockdown not due to increased cell death ............ 52

4.3 Involvement of the GATA transcription factor Tc-grain in labrum formation .......... 53

4.3.1 Identification of iB_03552 as Tc-grain (Tc-grn) ........................................... 53

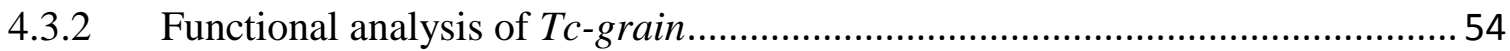

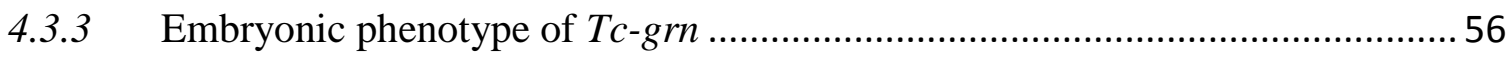

4.3.4 Tc-grn is expressed in the head throughout development................................ 57

4.3.5 Tc-grn is not an upstream regulator of the AMR network ................................. 60

4.3.6 Tc-grn is involved in the appendage regulatory gene network .........................61

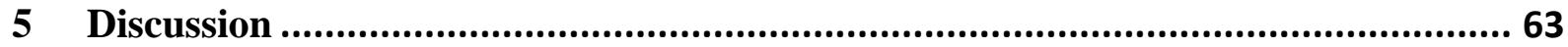

5.1 Low number of new head development genes detected in the iBeetle screen .......... 63

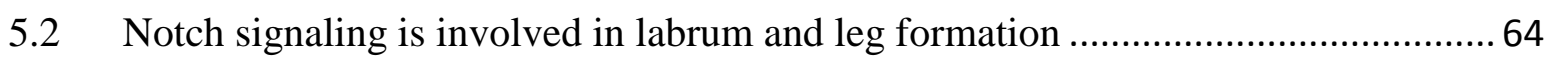

5.2.1 Conserved function of Notch signaling in arthropod leg development .............64

5.2.2 A novel role of Notch signaling in labrum formation .................................... 65

5.2.3 Notch signaling has a distinct role in the appendage regulatory network in the

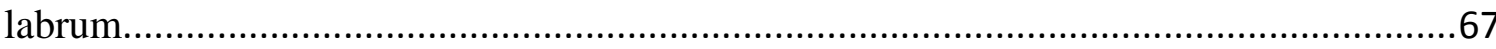

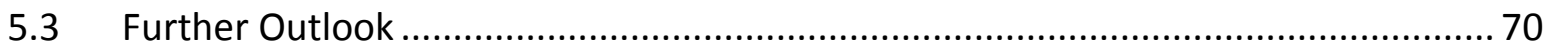


Table of contents

5.4 The role of the GATA transcription factor Tc-grn in labrum formation in Tribolium... 70

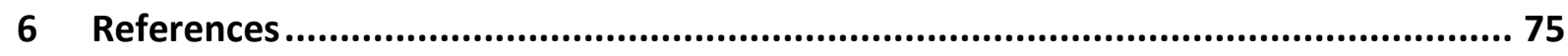

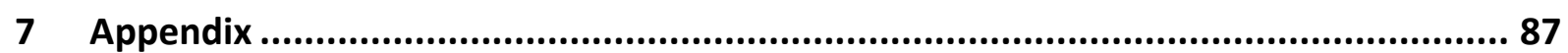

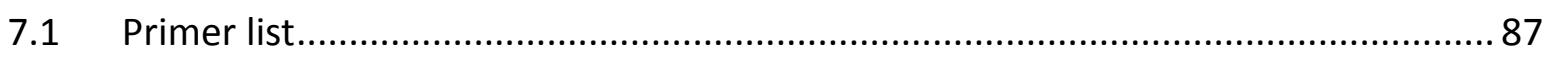

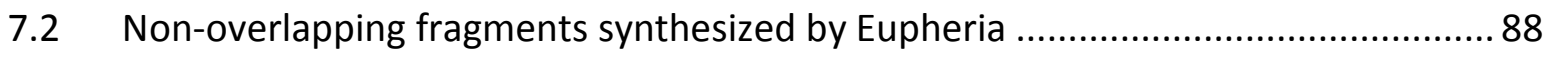

7.3 Number of proliferating cells in wild type and Tc-mib1 RNAi Embryos................... 88

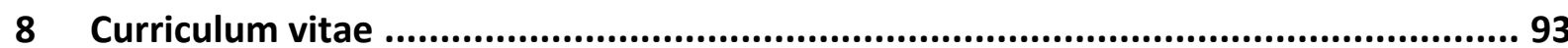




\section{Summary}

The insect head is a composite structure of several segments and an anterior non-segmental region. Due to head involution, the Drosophila larval head is strongly reduced and for technical reasons the developmental basis of head formation has not been comprehensively studied in Drosophila. In order to elucidate this process I used the red flour beetle Tribolium castaneum as model, focussing on the anterior median region (AMR) of the head, which harbors labrum and stomodeum. This region is patterned by a different set of genes than the surrounding tissues. The AMR development has already been studied using the candidate approach, which focused on genes previously identified in Drosophila. In order to identify a comprehensive list of genes involved in the formation of the AMR independent from previous knowledge, I used the unbiased large scale RNA interference screen (iBeetle-screen).

From the iBeetle results, I was able to identify three interesting genes which are required for proper labrum formation. Both Tc-Serrate and Tc-mibl are components of the Notch signaling pathway, which is known to be required e.g. for the formation of the joints and growth of adult legs and appendages in Drosophila and Tribolium. I showed that Tc-Ser is expressed during early AMR formation, but is not necessary for the early pattering process. During early labrum formation Notch signaling is required for the regulation of cell proliferation like in the Drosophila eye and wing imaginal discs. The labrum was suggested to be an appendage like structure, patterned by the same regulatory gene network. However, the results in this work indicate that Notch-signaling is acting upstream of the leg patterning network in the labrum in contrast to its function in the legs.

Another novel gene required for labrum development is the GATA transcription factor $T c$ grain. In Drosophila, grain is required for neurogenesis by regulating the axon guidance and in morphogenesis of the adult legs and the larval posterior spiracles. I showed that the expression of Tc-grn is conserved to Drosophila. Additionally, I showed that Tc-grn in Tribolium is likely to be involved in amnion development and is required for proper labrum formation probably by regulating morphogenesis. 


\section{Introduction}

\subsection{Segmental composition of the insect head}

The phylum of arthropods evolved the greatest species diversity within the animal kingdom (Mehlhorn, 1995) where the class of insects alone comprises more than a million described species. One of the main reasons of the evolutionary success is the subdivision of the body into single segments which allows for high morphological plasticity (Tautz, 2004). The body of insects is subdivided into three tagmata, i.e. head, thorax and abdomen. For example the heads evolved a great number of different forms each one of them adapted to a particular feeding style. While in some insects biting mouth parts are facing towards the ground (hypognathous) others evolved mouth openings directed to the anterior (prognathous) or posterior (opistognaghous). Nevertheless, all different head morphologies derived from one ancestral form (Snodgrass, 1935).

Furthermore, each tagma is built by a defined number of segments. While the number of segments building the thorax (three) and abdomen (eleven) is largely accepted (Snodgrass, 1935), the definition of the number of segments contributing to the insect head is disputed. Although data including classical morphological analysis but also molecular and genetic approaches were collected for the last decades, the number of segments and the possible existence of a non segmented region remains disputed (Budd, 2002; Diederich et al., 1991; Haas et al., 2001a; Haas et al., 2001b; Jürgens et al., 1986; Posnien et al., 2010; Rogers and Kaufman, 1996; Schmidt-Ott and Technau, 1994; Schmidt-Ott et al., 1994; Scholtz and Edgecombe, 2006).

Functionally the insect head is subdivided into a posterior region primarily involved in the feeding process (gnathocephalon) and an anterior region (procephalon) mostly involved in sensory processing. The gnathocephalon is formed by three segments including the labial, maxillary and mandibular segments (Snodgrass, 1935). The number of segments forming the procephalon is still under dispute but it is generally accepted that the intercalary and the antenna are true serially homologous segments. As they share a adjacent expression of the two segment polarity genes wingless ( $w g$ ) and engrailed (en), these five segments are seen as homologous to the trunk segments (Rogers and Kaufman, 1996; Schmidt-Ott and Technau, 1992). In addition, some authors also include the ocular region to the true segments (Rogers and Kaufman, 1996; Rogers and Kaufman, 1997). However, the term ocular/protocerebral 
region is preferred by others (Posnien et al., 2010; Scholtz and Edgecombe, 2006). The origin of the labrum is still enigmatic. During embryogenesis this structure arises as a pair of labral buds and fuses to form an unpaired structure located anterior to the mouth opening. Here it covers the mouth opening and is often called the insect upper lip (Rogers and Kaufman, 1997). For the last decades some authors argued that the labrum originated as an appendage of an anterior segment (Cohen and Jürgens, 1991; Finkelstein and Perrimon, 1991; Schmidt-Ott and Technau, 1992). Other suggested that the labrum is an appendage of the intercalary segment (Budd, 2002; Haas et al., 2001a; Haas et al., 2001b) or an integral part of the acron (Posnien et al., 2009a; Posnien et al., 2010; Rogers and Kaufman, 1997; Scholtz and Edgecombe, 2006). The acron is a putative non-segmental anterior head region. The acron concept originates from the assumption that arthropods and annelids share a common ancestor and that arthropods need to have a structure homologous to the anterior nonsegmantal prostomium of annelids(Scholtz and Edgecombe, 2006). New insights in molecular data showed that these two taxa are more distantly related (Eernisse et al., 1992). Anyway, the anterior regions of all bilateral animals are homologous (Arendt et al., 2008; Lowe et al., 2003; Reichert, 2009; Reichert and Simeone, 2001) and studies indicated the existence of an anterior head region which is not delimited by parasegment boundaries (Kittelmann et al., 2013; Posnien et al., 2009a). Nevertheless, the term "acron" is decrepit and was suggested to be replaced by ,anterior-non-segmental region“.

\subsection{Head patterning in insects}

The first insights into the metamerization process from a uniform into a fully segmented embryo originated in genetic and molecular studies in Drosophila. Analyses of mutant phenotypes affecting the segmental pattern in embryos revealed that a genetic cascade gradually subdivides the embryo into smaller compartments (Lewis, 1978; Nüsslein-Volhard and Wieschaus, 1980). First, the products of maternal effect genes give the initial positional information for the blastodermal axes (for example bicoid). In a concentration dependent manner these genes activate a cascade of zygotic genes, starting with the expression of gap genes. The gap genes define regional areas in the embryo which results in expression of pair rule genes. These genes are active in every other segment and thus subdivide the embryo into rough segments and activate segment polarity gene expression which defines the parasegment boundaries. The identity of each segment is defined by genes of the antennapedia and bithorax complex which are also known as Homeotic selector genes (Hox genes) (Akam, 1998; 
Ingham, 1988; St Johnston and Nüsslein-Volhard, 1992). The segments including the labial, maxillary and mandibular segments are patterned mostly like the trunk, whereby the latter includes also input of the head gap-like genes (Vincent et al., 1997). However, the procephalon is patterned in a different way. Expression of a Hox cluster gene is only present in the posterior most region of the procephalon, the intercalary segment (labial/Hoxl) (Abzhanov and Kaufman, 1999; Diederich et al., 1989; Diederich et al., 1991; Merrill et al., 1989; Posnien and Bucher, 2010). In contrast, the most anterior region including labrum, eyes and antenna are completely free of Hox cluster genes. In addition, the procephalon is patterned without pair rule genes while segment polarity genes require the action of head gaplike genes (Cohen and Jürgens, 1990; Crozatier et al., 1999; Grossniklaus et al., 1994; Wimmer et al., 1997). These head gap like genes include genes like orthodenticle, emptyspiracles, buttonhead (Cohen and Jürgens, 1990; Cohen and Jürgens, 1991) and sloppy paired (Grossniklaus et al., 1992).

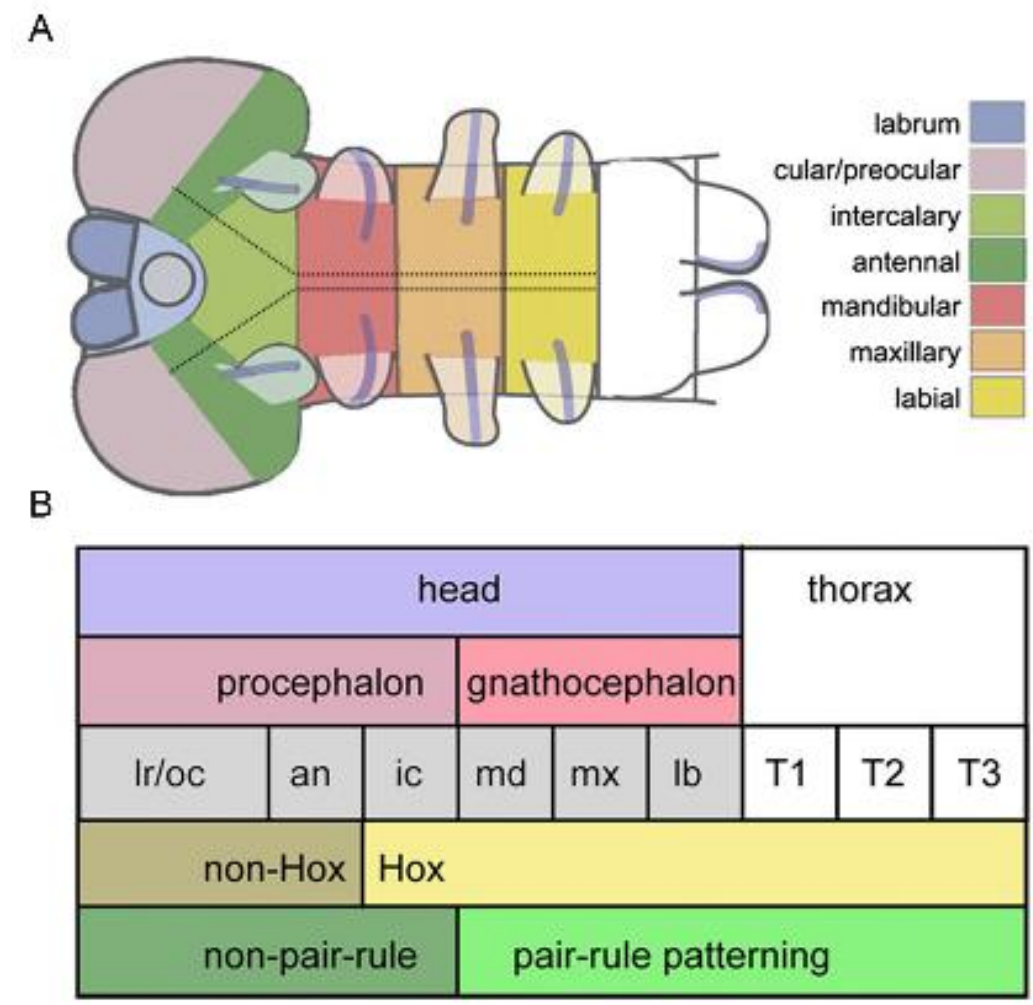

Figure 2.1: Composition and patterning of the insect head - (A) Schematic view of an elongating embryo. (B) Schematic subdivision of the head and pattering processes. The anterior head (procephalon) compromise labrum, ocular region, antennal and intercalary segments. The posterior part (gnathocephalon) is subdivided into mandibular, maxillary and labial segments. The dotted line represents the ventral midline of neurogenic genes including the anterior split (A). Formation of the procephalon occurs without pair rule patterning and Hox genes are only expressed up to the intercalary segment. The gnathocephalon is mostly patterned like the trunk segments 
(B). labrum (lr); ocular region (oc); antennal (an); intercalary (ic); mandibular (md); maxillary (mx); labial (lb). Images taken from (Posnien et al., 2010).

Despite the fact that the anterior and the posterior head regions are regulated by different segmentation cascades and some genes and interactions could be identified, a comprehensive understanding of the anterior pattering process is not available in Drosophila so far.

\subsection{Using Tribolium castaneum as a model for insect head patterning}

Drosophila melanogaster is the most studied insect model organism which provides powerful tools to understand the genetic basis of development. Unfortunately, in order to get a comprehensive understanding of the regulatory network for insect head development Drosophila is not the best suited model organism. Beside the fact that Drosophila undergoes long germ embryogenesis where segments are formed simultaneously in early blastoderm (St Johnston and Nüsslein-Volhard, 1992), the gene bicoid which is involved in the establishment of the anterior portion of the embryo could not be found in insects other than dipterans so far (Brown et al., 2001; Stauber et al., 1999). In addition, during late embryogenesis the main head structures undergo a morphogenetic event which causes internalization of the larval head into the thorax (Jürgens et al., 1986; Turner and Mahowald, 1979; VanHook and Letsou, 2008; Younossi-Hartenstein et al., 1993). This process is called head involution and results in a highly derived and reduced head structure in larvae. In addition to the fact that this process reflects not the insect-typical head development since it only occurs in higher dipterans (Younossi-Hartenstein et al., 1993), its reduction results in poor morphological markers. Beyond that, mutations of head pattering genes are not easy to interpret because head involution is often disrupted which causes secondary defects (Merrill et al., 1989).

In contrast to that, the red flour beetle Tribolium castaneum reflects are more ancestral mode of arthropod segmentation and development (Bucher and Wimmer, 2005; Davis and Patel, 2002; Klingler, 2004; Schoppmeier and Schröder, 2005; Schröder et al., 2008; Tautz et al., 1994). One important fact is that Tribolium undergoes short germ embryogenesis. Here, anterior segments are specified during early blastoderm stages, while posterior segments are added step-by-step from the posterior growth zone of the embryo (Davis and Patel, 2002; Sarrazin et al., 2012; Schoppmeier and Schröder, 2005; Tautz et al., 1994). Furthermore, Tribolium develops a larval head with external insect typical and well distinguishable structures (Bucher and Wimmer, 2005). In addition, a characteristic bristle pattern facilitate the identification of certain head regions (Posnien and Bucher, 2010; Schinko et al., 2008). 
Moreover, a growing tool set for functional analysis was developed over the last years. Beside an accessible genome (Richards et al., 2008), a robust RNA interference (RNAi) method is available to knockdown gene function. In particular the response is transmitted systemically allowing manipulation of genes at every stage of development because dsRNA can be easily injected in pupae or adult and the effect is even transmitted to the offspring (Brown et al., 1999; Bucher et al., 2002; Tomoyasu and Denell, 2004; Tomoyasu et al., 2008). In addition, a piggyBag based transgenic insertional mutagenesis screen provided enhancer trap lines marked with EGFP (Berghammer et al., 1999; Trauner et al., 2009). Moreover, imaging lines were produced (Posnien et al., 2011b; Sarrazin et al., 2012) and misexpression techniques were established (Schinko et al., 2010; Schinko et al., 2012).

\subsection{Direct comparison of Drosphila and Tribolium head patterning}

As already mentioned, the head development of Drosophila is derived within insects. Indeed the patterning of the anterior region of the head is not highly conserved in direct comparisons to Tribolium. The upstream regulator bicoid of the head gap like genes is completely missing outside higher dipterans (Brown et al., 2001; Lemke et al., 2008; Stauber et al., 1999) but Tcaxin mRNA is required for anterior development (Fu et al., 2012) and also the head gap like genes itself show great functional variation. While Tc-buttonhead has no role at all, the function of Tc-empty spiracles is restricted to the posterior ocular and anterior portion of the antennal segments. The gene Tc-orthodenticlel has an additional role in axis formation (Schinko et al., 2008; Schröder, 2003) and the genes Tc-huckebein and Tc-tailless are not involved in head development (Kittelmann et al., 2013; Posnien et al., 2011b; Schoppmeier and Schröder, 2005; Schröder et al., 2000). In contrast, other genes which function in a different developmental context in Drosophila showed essential function in head development, for example the gene Tc-knirps (Cerny et al., 2008). However, more downstream genes like cnc, croc and collier show conserved expression and function (Coulcher and Telford, 2012; Economou and Telford, 2009; Kittelmann et al., 2013; Schaeper et al., 2010).

\subsection{The labrum arises in the anterior median region (AMR)}

Due to the difference to trunk patterning, the anterior procephalic region is especially interesting. The labrum is located in the non-segmental anterior-median region which is 
bordered by neuroectoderm. While neurogenic genes (for example Tc-mae, Tc-spitz, Tcasense and Tc-achaete-scute) are expressed as two separated stripes along the ventral midline, they split in the anterior head outwards, thereby shaping a "Y" (dotted line in Figure 2.1A). The median tissue framed by neuroectoderm is called anterior median region (AMR) and gives rise to the labrum and stomodeum (Bucher and Klingler, 2005; Kittelmann et al., 2013; Wheeler et al., 2003). In addition, the expression of the segment polarity gene Tc-wingless $(T c-w g)$ shows this $\mathrm{Y}$ shaped pattern framing the AMR. While the stripes of $T c-w g$ are perpendicular to the body axis, antennal and ocular domains are twisted outwards (Posnien et al., 2010). This region also expresses a specific set of genes (Tc-six3, Tc-crocodile (Tc-croc), Tc-cap'n'collar (Tc-cnc), Tc-scarecrow (Tc-scro), and Tc-forkhead (Tc-fkh) which is expressed inside the AMR but not in each segment. This indicates that the AMR is patterned by another genetic regulatory network than the surrounding tissue (Economou and Telford, 2009; Posnien et al., 2009a; Posnien et al., 2011b; Schröder et al., 2000). Indeed functional analysis showed that knockdown of Tc-six3 led to deletion of this region but the surrounding tissue remained largely unaffected (Posnien et al., 2009a; Posnien et al., 2011b). Tc-six3 is required for early AMR patterning and acts upstream of Tc-cnc and Tc-croc. While Tc-cnc is involved in labrum formation in the anterior AMR, Tc-croc is required for stomodeum development in the posterior AMR (Kittelmann et al., 2013).

The labrum structure itself requires the genetic regulatory network of appendage development. In situ staining of appendage patterning genes revealed expression in a similar relative position in both labrum and trunk segments including the genes $T c$-wg, Tc-Distal-less (Tc-Dll), Tc-decapentaplegic (Tc-dpp), Tc-homothorax (Tc-hth) and Tc-dachshund (Tc-dac) (Beermann et al., 2001; Nagy and Carroll, 1994; Posnien et al., 2009a; Prpic et al., 2001; Sanchez-Salazar et al., 1996). Moreover, functional analysis of $T c-D l l$ indicated that it is both involved in appendage and labrum patterning, since distal portions of the labrum and appendages are deleted in Tc-Dll (Short antennae (Sa)) mutants (Beermann et al., 2001). Conversely, recent studies indicated that the regulatory gene network of the appendages function in a different way in the labrum because activation of $T c-D l l$ depends on $T c-w g$ and Tc-hedgehog $(T c-h h)$ in the appendages but not in the labrum (Posnien et al., 2009a). Therefore the labrum has been suggested to be an appendage like structure, but not located in the segmental part of the embryo. 


\subsection{Identification of novel head patterning genes}

So far, the study of head development has relied on candidate genes known for Drosophila and vertebrates. Based on these genes the understanding of the regulatory network of the anterior median region including the patterning of the labrum was expanded over the last years. However, there is still no comprehensive set of genes available that are required for AMR patterning. For example, the mode of activation of $T c$-six3 is still enigmatic and upstream activators of the posterior AMR are missing (Kittelmann et al., 2013). In order to find novel genes required for AMR patterning, genes showing a head phenotype in a genome wide RNAi screen (iBeetle screen) were identified in this work.

The iBeetle screen uses the knockdown of randomly selected genes via RNAi to identify novel gene functions in an unbiased way. Here, two screens were performed in parallel at different developmental stages to allow the identification of novel gene functions during oogenesis, embryogenesis, and metamorphosis. In the "pupal injection screen", injected female pupae were scored for late metamorphosis phenotypes and their offspring were analyzed for both muscle and cuticle phenotypes. In addition, ovaries were analyzed for oogenesis defects, if reduced egg production was observed. The "larval injection screen", focused on the insect metamorphosis and development of the odoriferous glands by injection in L5/6 larvae. In the first screening period 5,300 genes in the "pupal" and 4,480 genes in the "larval injection screen" were screened (Schmitt-Engel et al., accepted). The RNAi phenotypes of both screens are annotated in an online database available at http://ibeetlebase.uni-goettingen.de/ using the vocabulary based on the Tribolium morphological ontology (TrOn) (Dönitz et al., 2013; Dönitz et al., 2014).

\subsection{The developmental function of Notch signaling}

During this work, two genes were analyzed, which showed a labrum specific phenotype in the screen. Both genes were components of the Notch signaling pathway. In Drosophila the Notch signaling pathway is involved in a wide variety of cell fate decisions and other developmental processes (Artavanis-Tsakonas et al., 1999; Lai, 2004). A well characterized function of Notch is those affecting neurogenesis in flies and vertebrates by mediating lateral inhibition of neural precursors (neuroblasts) (Artavanis-Tsakonas et al., 1999; Cabrera, 1990; Martín-Bermudo et al., 1995; Simpson, 1990).

Moreover, the Notch signaling pathway interacts with other pathways for instance with the Wnt signaling pathway in wing margin formation (Axelrod et al., 1996; Diaz-Benjumea and 
Cohen, 1995; Hing et al., 1994). Further, Notch is also involved in regulating cell proliferation. For instance, by control of cell proliferation eye primordium formation and wing disc grow are influenced (Go et al., 1998; Kenyon et al., 2003; Speicher et al., 1994). In leg formation, Notch signaling plays a role in growth control and is also specifying the location and number of leg joints. Starting with proximal-distal patterning of the leg disc induced by $w g$ and $d p p$ (Figure 2.2A), this genes act together to regulate the expression of the leg gap genes homothorax (hth), dac and Dll (Figure 2.2B) (Abu-Shaar and Mann, 1998; Brook et al., 1996; Duncan et al., 1998; Lecuit and Cohen, 1997; Wu and Cohen, 1999). In a next step these genes establish the expression of leg segmentation genes in ring shaped domains including genes of the Notch signaling pathway, i.e. Serrate and Delta (Figure 2C) (Bishop et al., 1999; Rauskolb, 2001; Rauskolb and Irvine, 1999).

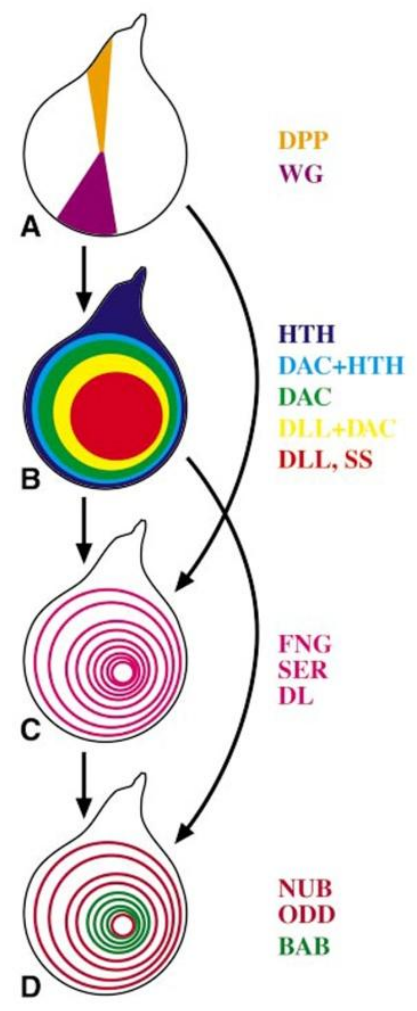

Figure 2.2: Leg segmentation in Drosophila - (A) Proximaldistal axis patterning in the leg imaginal disc is promoted by the combined action of $w g$ and $d p p$. (B) These genes regulate the expression of the genes $h t h, d a c$, Dll, and spineless (ss). (C) In a combinatorial action of these genes rings of expression of $f n g$, Ser, and $D l$ are established (Rauskolb, 2001), possibly also with direct input from $w g$ and dpp. (D) Notch signaling and genes at higher levels of the hierarchy, influences the expression of e.g, nub, odd and presumpably bab (Godt et al., 1993). Schema taken from (Rauskolb and Irvine, 1999). 


\subsubsection{The Notch pathway}

Components of the Notch signaling pathway are well characterized in invertebrates and vertebrates, including humans. Since The Notch gene was first characterized in Drosophila melanogaster (Kidd et al., 1986; Wharton et al., 1985), the number of identified proteins which are involved in transmission or regulation of the Notch signal increased (ArtavanisTsakonas et al., 1999). To allow cell-to-cell communication between neighboring cells, the interaction of two transmembrane proteins is needed. Active signaling is provided if a signal sending cell expresses the ligand Delta or Serrate (in vertebrates Serrate is called Jagged (Fleming, 1998)) on their surface, and comes in contact with the extracellular domain of the Notch receptor of a signal receiving cell (Artavanis-Tsakonas et al., 1999). Both ligands and receptor contain extracellular domains with epidermal growth factor (EGF)- like repeats (Rebay et al., 1991) which are necessary to bind to each other. If an activated ligand binds to the receptor, two proteolytic cleavages are induced. The first cleavage is catalyzed by ADAM-familiy metalloproteases (Pan and Rubin, 1997), while the second is promoted by gamma-secretase (Figure 2.3, S2 and S3) (Fortini, 2002; Selkoe and Kopan, 2003). After the second cleavage the Notch intracellular domain (Nicd) translocates to the nucleus where it promotes transcription after interaction with a DNA binding protein CSL $(\underline{C B F} 1, \underline{\operatorname{Su}}(\mathrm{H})$ and LAG-1) and its co activator Mastermind (Mam) (Borggrefe and Oswald, 2009; Bray, 2006; Fortini, 2002; Fryer et al., 2004; Mumm and Kopan, 2000; Struhl and Adachi, 1998). 


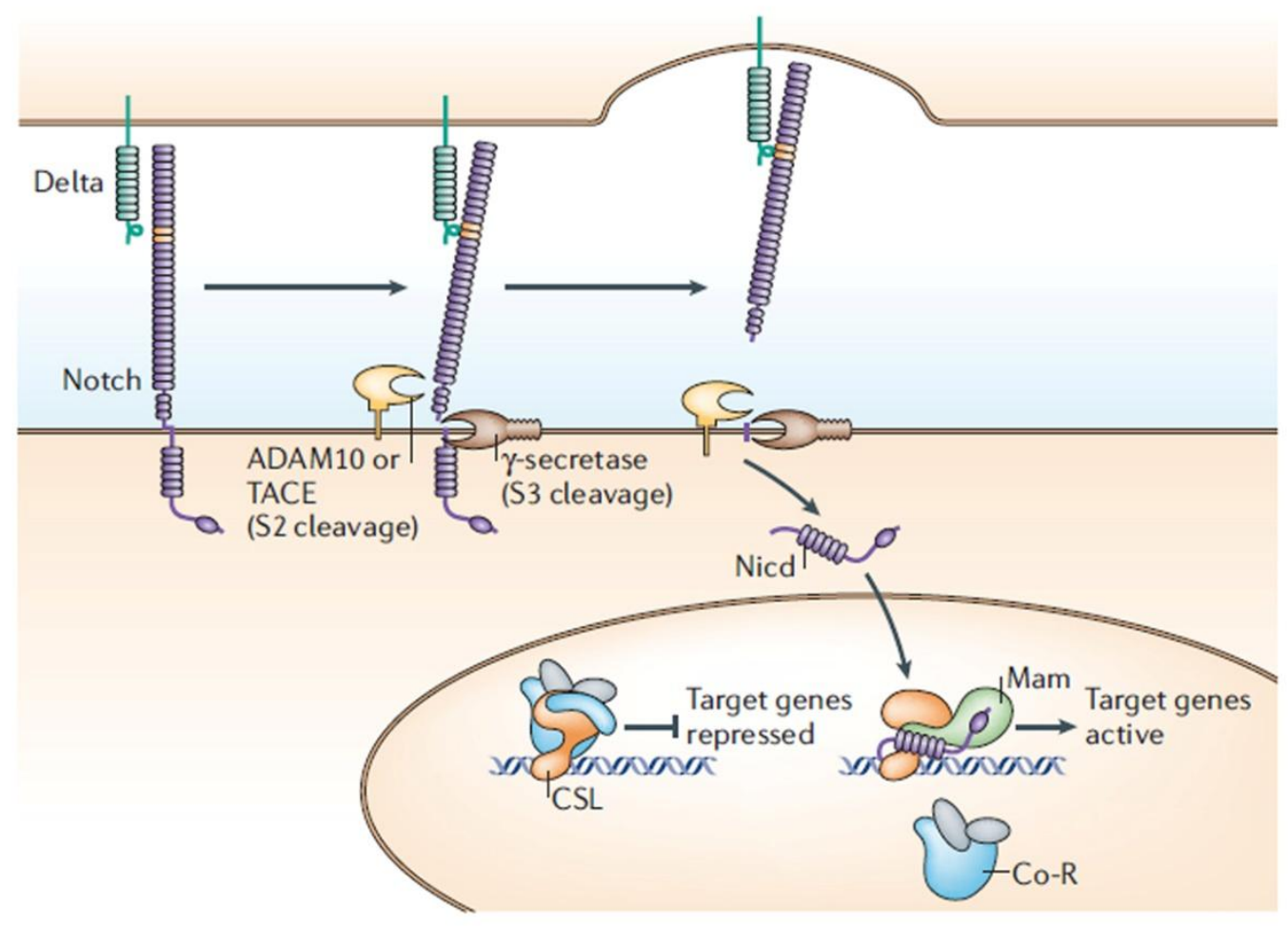

Figure 2.3: The Notch signaling pathway. Ligand binding of Delta (green) or Serrate (not shown) of the signal sending cell to the Notch receptor of another cell results in two proteolytic cleavages (S2, S3). ADAM metalloproteases catalyze the S2 cleavage while S3 is catalyzed by gamma-secretases. After cleavage the Notch intercellular domain (Nicd) enters the nucleus and interacts with DNA-binding Protein (CSL) and its co-activator Mastermind (mam) to promote transcription. Additionally, co-repressors (Co-R; blue and grey) are released. Scheme from (Bray, 2006).

\subsubsection{Regulation of Notch signaling}

While simple at first, Notch signaling becomes very complex when its regulation is considered. As already mentioned, Notch signaling requires activation of its two ligands Delta and Serrate. For a long time endocytosis of ligands and receptors was thought to induce downregulation of cell-cell signaling (for instance targeting receptors for lysosomal degradation). However, new insights estimated that endocytosis might also be required for signal transduction (Klueg and Muskavitch, 1999; Kooh et al., 1993; Le Borgne et al., 2005a; Parks et al., 2000; Seugnet et al., 1997). For the last years, several models have been proposed to answer the question how endocytosis is associated with receptor activation but it is still not fully understood (Le Borgne and Schweisguth, 2003a; Le Borgne et al., 2005a; Parks et al., 2000; Wang and Struhl, 2004). Recent investigations have described that ubiquitination plays a key role in endocytosis of the ligands by the two E3 ubiquitin ligases Neuralized (Neur) and 
Mind bomb 1 (Mib1) in vertebrates and invertebrates (Artavanis-Tsakonas et al., 1999; Chen and Casey Corliss, 2004; Itoh et al., 2003; Lai, 2004; Schweisguth, 2004). Previously, it was thought that ubiquitin targets proteins for their destruction in proteasomes. However, recent insights showed that it also plays a role in endocystosis and activation of Notch ligands (Figure 2.4) (Bonifacino and Weissman, 2002; Hicke, 2001; Weissman, 2001). The multistep process of ubiquitination includes an addition of an ubiquitin, composed of 76 amino acids, to a substrate protein. Beside the E3 ligases this process involves two other classes of enzymes including E1 (ubiquitin-activating enzymes) and E2s (ubiquitin-conjugating enzymes) (Bonifacino and Weissman, 2002).

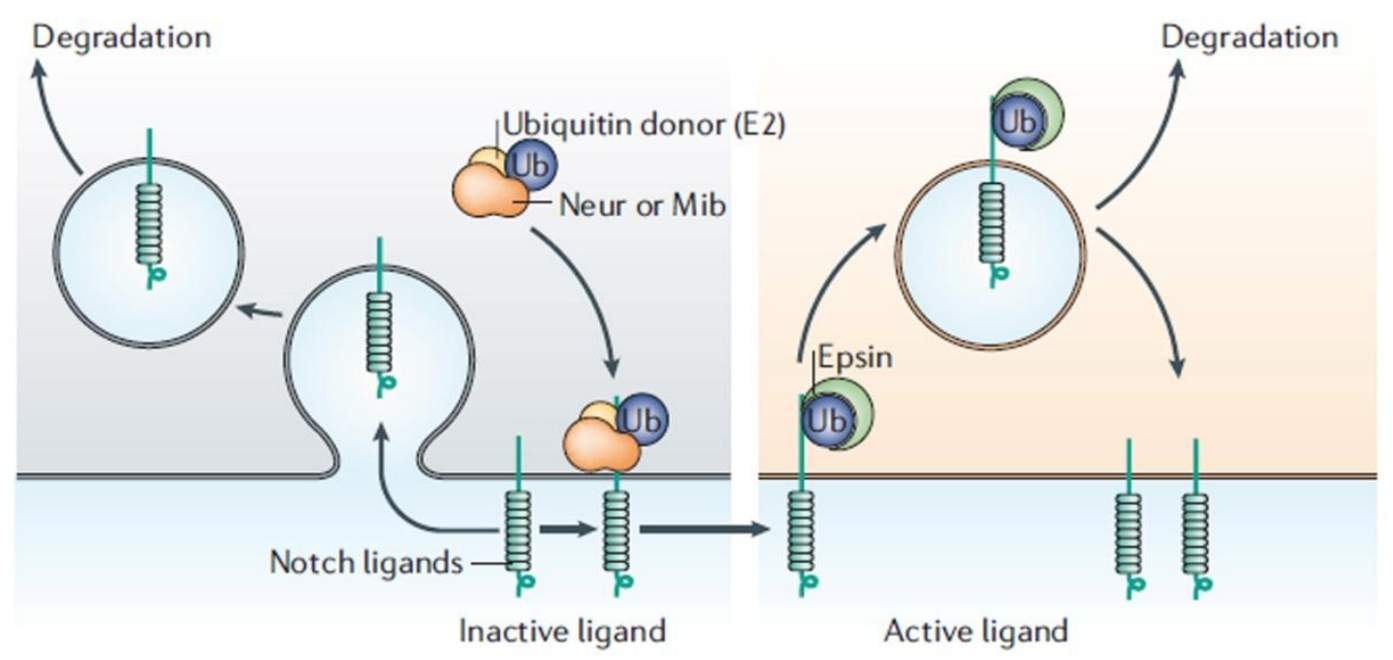

Figure 2.4: Ligand activation via ubiquitination - While the ligands Delta and Serrate are inactive, they can be either endocytosed or degraded. Neur and Mib (Ub) mediated ubiquitination of Notch ligands is required for Epsin mediated endocytosis. After endocytosis ligands are able to signal but can also be targeted for degradation. Figure from (Bray, 2006).

Neur and mibl are related to distinct ligase families. Thus, differ in their primary structure but show sequence homology (C-terminal RING fingers) and similarity in their activities (Lai et al., 2005; Le Borgne et al., 2005b; Wang and Struhl, 2005). Both E3 ligases can directly interact with Delta and Serrate (Daskalaki et al., 2011; Glittenberg et al., 2006) to allow endocytosis and are interchangeable to some degree. Nevertheless, it appears that they have 
distinct developmental functions in Drosophila. While neur shows a restricted expression in sensory cells (Boulianne et al., 1991), mibl is uniformly expressed in imaginal dics (Lai et al., 2005; Le Borgne et al., 2005b). Furthermore, recent data showed that mibl is required for Notch events which do not need neur gene activity. Neur is required for lateral inhibition during neurogenisis (Lai and Rubin, 2001; Lehmann et al., 1983; Yeh et al., 2000) and is also involved in cell fate decisions in the bristle lineage (Le Borgne and Schweisguth, 2003). The latter does not need mibl activity (Le Borgne et al., 2005b). Thus, Drosophila mibl was missed in earlier screens for Notch pathway components, because the related phenotypes were only weakly neurogenic. In contrast mibl is involved in Notch signaling events where neur is not needed, like wing margin formation, leg segmentation and vein formation (Lai et al., 2005; Le Borgne et al., 2005b; Wang and Struhl, 2005). Additionally, Mib1 might prefer Serrate as a substrate because $D$-mibl mutant cells showed that Mib1 is required for Serrate but not Delta endocytosis (Le Borgne et al., 2005b; Wang and Struhl, 2005) and Serrate accumulation defects occur in imaginal disc (Lai et al., 2005).

\subsubsection{The Notch signaling pathway in Tribolium}

In Tribolium the Notch signaling pathway is also involved in different processes during development. Here, Notch and its ligand Delta have a major role during telotrophic oogenesis, which explains the high sterility after $N$ and $D l$ RNAi (Baumer et al., 2012). Moreover, it was previously shown that downstream targets of Delta-Notch signaling (bHLH-O repressors $E(s p l) 1$ and $E(s p l) 3)$ mediate lateral inhibition in the neuroectoderm (Kux et al., 2013). In addition, a role for Notch signaling in growth control of the leg was suggested before, since knockdowns of Tc-Delta resulted in shortened legs compared to the wild type (Aranda, 2006). Furthermore, Tc-Serrate is expressed in rings in the legs, marking the positions of the leg joints. This gave evidence for the involvement of Notch signaling in joint formation (Beermann et al., 2004). During metamorphosis, joint formation in the antenna and legs as well as metamorphosis of the maxillary endites requires the activation of Notch signaling. Here, it is also involved in growth control of the legs (Angelini et al., 2009; Angelini et al., 2012a; Angelini et al., 2012b). Additionally, the sensory patterning in the labrum during metamorphosis is linked to the Notch signaling pathway (Smith et al., 2014). 


\subsection{The GATA transcriptions factor grain}

In Drosophila, three genes pannier (pnr), serpent (srp) and grain (grn) encode GATA transcriptions factors. The gene $p n r$ plays a role in the development of dorsal ectoderm derivatives and extraembryonic tissue where it is involved in the $d p p$ signaling pathway (Heitzler et al., 1996; Ramain et al., 1993; Winick et al., 1993). The gene srp is known to be involved in the development of the gut and the fat body (Abel et al., 1993; Rehorn et al., 1996; Reuter, 1994). In recent years also grn was further analyzed and revealed that this gene is involved in different developmental processes. This includes a role in neurogenesis by regulating the axon guidance due to activation of the unc-5 in dorsal motoneurons (Garces and Thor, 2006; Zarin et al., 2012; Zarin et al., 2014). In addition, it also plays a role in cell rearrangement during morphogenesis (Brown and Castelli-Gair Hombría, 2000). Here grn is involved in shaping the adult legs and the larval posterior spiracles controlling cell rearrangements in the embryo and imaginal discs. Interestingly, this study also showed an involvement in head development. A localized expression domain of grn starts during late blastoderm stages in the procephalon and cuticle analysis revealed that the head cuticle has defects mostly in the dorsal bridge and in the lateralgräten. These structures belong to the anterior non segmental part of the embryo and the mandibular segment (Jürgens et al., 1986).

\subsection{Aims}

The main aim of this work was to find and characterize novel insect head patterning genes with focus on the anterior median region. This included three different steps. First, potential patterning genes were selected from the iBeetle screen which showed head phenotypes after knockdown. Second, all selected candidate gene were rescreened to check for reproducibility and specifity. Finally, the most promising reproduced candidates were selected for more detailed analysis.

Based on the expression and function of the Tc-mibl and Tc-Ser, I hypothesised the hierarchy of Notch signaling within the leg patterning network in the labrum and the leg. This was mostly tested by analysis of marker genes after knockdown. Additionally, I analyzed Notch mediated cell proliferation in proper labrum and leg formation.

Since Tc-grn knockdown indicated to be involved in labrum formation, I used expression and functional analysis to gain insight into the role of Tc-grn in Tribolium. Finally, I compared the data for Tc-grn in Tribolium with the corresponding expression and functional data of Drosophila in order to hypothesise the function of Tc-grn in labrum formation. 


\section{Material and methods}

\subsection{Stock keeping}

Strains of Tribolium castaneum were reared under standard conditions on full grain flour supplemented with $5 \%$ yeast at $32^{\circ} \mathrm{C}$ or $25^{\circ} \mathrm{C}$ (Brown et al., 2009). For experiments the wild type strain San Berndardino or the strain Pig-19 (pBa19) were used. Female pupae of the Pig19 strain were mated to males of the Black strain (Sokoloff et al., 1960) for an easily discrimination between injected females and not injected males. For egg collections beetles were kept on white flour provided with $5 \%$ dry yeast at $32^{\circ} \mathrm{C}$.

\subsection{Candidate gene selection}

To identify novel head patterning genes potential candidates were selected based on head phenotypes found in the iBeetle screen. Phenotypes were searched on the iBeetle database (http://ibeetle-base.uni-goettingen.de/) (Dönitz et al., 2015) using data gathered at day 15 (pd15) of the pupal injection screen. The following search terms were used:

"pd15, head, labrum"

"pd15, vertex triplet"

"pd15, head capsule"

“pd15, procephalic head"

To eliminate non head specific phenotypes all results were in addition searched for defects of the entire cuticle and embryos which did not form a cuticle. Such datasets were removed. In order to avoid missing interesting phenotypes head phenotypes were not filtered for low penetrance.

\subsection{Orthology and phylogenetic analysis}

In order to retrieve the gene sequences of the targeted genes, the corresponding ibeetle ID number (e.g. iB_05634) was searched in the Tribolium gene browser (http://bioinf.uni- 
greifswald.de/gb2/gbrowse/tribolium/). Orthologs were identified by BLAST analysis using translated nucleotide sequences against non-redundant protein sequences of Drosophila melanogaster and Mus musculus with the blastp algorithm on the NCBI database (National Center for Biotechnology Information NCBI, http://blast.ncbi.nlm.nih.gov/Blast.cgi (BLAST; (Altschul et al., 1990)). The protein sequences of the three best hits were used for alignments and phylogentic trees. Potential paralogs of Tribolium were obtained using the BeetleBase BLAST (http://www.Beetlebase.org; (Kim et al., 2010; Tribolium Genome Sequencing Consortium et al., 2008; Wang et al., 2007)). All protein sequences were aligned using clustlW alignment algorithm implemented in MEGA4 (http://www.megasoftware.net/mega4/mega.html) or Geneious 7 software (Biomatters, Auckland, New Zealand) using standard settings. Phylogenetic analysis was done using the Geneious Tree Builder with the Jukes-Cantor genetic distance model. As tree-building method the neighbor-joining (Saitou and Nei, 1987) method was used. Details for each candidate gene were retrieved by searching for the identified orthologs of Drosophila on flybase (http://flybase.org/) (McQuilton et al., 2012).

\subsection{Molecular biology}

Gene specific primers were designed based on AUGUSTUS gene predictions (AUGUSTUS UTR and hints from cDNA) from a project internal Tribolium gene browser. Primer synthesis was performed by Eurofins MWG Operon (Ebersberg, Germany). Genes of Tribolium were cloned following standard methods (Sambrook and Russel, 2001) from complementary DNA (cDNA) of 0-72 h old embryos using gene specific primers. cDNA was synthesized by Jonas Schwirz with the SMART PCR cDNA kit (ClonTech). Respective gene fragments were amplified using the Phusion chain polymerase and cloned into the pJET1.2 vector. A number of clones was generously provided from the clone collection of the department or other laboratories (Table 3.1). Cloned gene fragments were sequenced by Macrogen (Seoul, Korea and Amsterdam, Netherlands) using standard pJet Primer (pJET1.2 Forward Sequencing Primer, 23-mer 5'-CGACTCACTATAGGGAGAGCGGC-3' and pJET1.2 Reverse Sequencing Primer, 24-mer 5'-AAGAACATCGATTTTCCATGGCAG-3'). All gene specific primer generated in this thesis are listed in the appendix. 


\begin{tabular}{|c|c|c|c|}
\hline Clone & Provided by & Length & Used for \\
\hline Tc-Delta & Manuel Aranda & $500 \mathrm{bp}$ & Probe preparation \\
\hline$T c$-Serrate & Manuel Aranda & $610 \mathrm{bp}$ & Probe preparation, dsRNA synthesis \\
\hline$T c$-Dll & Nico Posnien & $948 \mathrm{bp}$ & DsRNA synthesis \\
\hline$T c$-dac & Martin Klingler & $950 \mathrm{bp}$ & Probe preparation \\
\hline Tc-grain & Cristina De Miguel Vijandi & $588 \mathrm{bp}$ & Probe preparation \\
\hline
\end{tabular}

Table 3.1: cDNA clones provided by other people. Indicated is the name of the gene, the provider, the length of the clone and what they were used for in this work.

\subsection{Knock down by RNA interference (RNAi) and injection techniques}

Knockdown of gene function in Tribolium castanuem was performed according to previously published protocols (Posnien et al., 2009b) by injection of double stranded RNA (dsRNA) into pupae. Required templates for in vitro transcription were amplified via PCR using primers with an attached T7 polymerase promoter sequence (pJET_fw-upT7 5'ACACTTGTGCCTGAACACCATACC-3' and pJET12_rev_T7 5'TAATACGACTCACTATAGGAAGAACATCGATTTTCCATGGCAG-3'). The generation of dsRNA was done using the Ambion ${ }^{\circledR}$ MEGAscript ${ }^{\circledR}$ T7 kit (Life Technologies, Carlsbad, CA, USA). To test for off-targets (unspecific knockdown due to sequence homologies) of the genes of interest, non-overlapping fragments were generated for each gene. The cDNA sequences of the final selection of candidate genes used for dsRNA synthesis were also analyzed using E-RNAi on the website of the German Cancer Research Center (DKFZ) (http://www.dkfz.de/signaling/e-rnai3/) (Horn and Boutros, 2010). Moreover, most fragments were injected both in the $S B$ and the Pig-19 strain to check whether the fragments exhibit strain specific phenotypes. Only fragments showing the same phenotype in both strains were used for final experiments. For the rescreen analysis dsRNA at a concentration of $1 \mu \mathrm{g} / \mu 1$ and $3 \mu \mathrm{g} / \mu \mathrm{l}$ was injected into female pupae and for ongoing experiments a concentration of $1 \mu \mathrm{g} / \mu \mathrm{l}$ up to $2 \mu \mathrm{g} / \mu \mathrm{l}$ of dsRNA was chosen. Buffer injections without dsRNA were performed as control. All injections into female pupae were done using a FemtoJet ${ }^{\circledR}$ express device (Eppendorf, Hamburg, Germany) with an applied injection pressure of 300-700 hpc. Embryonic injections were performed according to published protocol (Berghammer et al., 2009) using a FemtoJet® express device (Eppendorf, Hamburg, Germany). 


\subsection{Histology}

\subsubsection{Probes}

In situ probes were synthesized with the DIG (Digoxigenin) and FLU (Fluorescein) labeling kits from Roche using T7 polymerase for all genes cloned into the pjet1.2 vector and for the probe preparation of the provided clones of Tc-grain (pST Blue-1) and Tc-Dl (pBluescript). The T3 polymerase was used for probe synthesis of Tc-Ser (pBluescript) and Tc-dac (pCR Script).

\subsubsection{Egg collections and fixation}

For in situ hybridization (ISH) and immune histochemistry embryos of an age between 0-48 $\mathrm{h}$ were used. For staining of the cell membranes with $F M{ }^{\circledR}$ 1-43 eggs of 0-69 h were collected. For cell proliferation assay 19-20 h old embryos were used. If not mentioned otherwise fixations of embryos were done according to given standard protocols (Schinko et al., 2009). Fixation buffer was modified after (Sandmann et al., 2006) 1mM EDTA, 0.5mM EGTA, $100 \mathrm{mM} \mathrm{NaCl}, 2.5 \%$ formaldehyde, 50mM HEPES, $\mathrm{pH}$. The embryos were agitated for a maximum of 25 minutes at $220-230 \mathrm{rpm}$.

\subsubsection{Whole mount in situ hybridization (ISH)}

ISH was done with an alkaline phosphatase driven Nitro blue tetrazolium (NBT) and 5Bromo-4-chloro-3-indolyl phosphate (BCIP) reaction using described protocols (Schinko et al., 2009). Staining was also performed after the protocol described previously (Oberhofer et al., 2014).

Double in situ hybridization was performed using NBT/BCIP and a horseradish peroxidase (POD) mediated tyramide signal amplification (TSA). The TSA tyramide Dylight 550 conjugate was used as substrate (synthesized by Georg Oberhofer). Staining was performed with anti-Dig-AP (Roche) for the NBT/BCIP and anti-Fluo-POD (Roche) for the TSA reaction (Oberhofer et al., 2014; Schinko et al., 2009).

Embryonic nuclei were detected using 0,6ng/ $\mu$ l Hoechst 33342 during the last washing steps, followed by at least two washing steps with PBT. 


\subsubsection{FM@ 1-43 staining}

Fixed embryos were rehydrated in 1:1 methanol/PBT and washed for two times with PBT Staining was done using 0,0005 $\mathrm{ng} / \mu \mathrm{l} \mathrm{N}$-(3-triethylammoniumpropyl)-4-(4-(dibutylamino) styryl)pyridinium dibromide (FM® 1-43; Invitrogen/Life Technologies, Carlsbad, CA, USA) for 30 minutes. Afterwards embryos were washed for at least 3 washing steps with PBT. Staining of nuclei was performed with Hoechst.

\subsubsection{Cleaved Drosophila death caspase-1 (Dcp-1) staining}

For antibody staining with the cleaved Drosophila Dcp-1 (Asp216) antibody (Cell Signaling Technology) fixed embryos were rehydrated in 1:1 methanol/PBT and then washed two times in PBT. After another two washing steps of about 15 minutes each, embryos were blocked for at least one hour in $1 \mathrm{ml} \mathrm{3 \%} \mathrm{BSA} \mathrm{in} \mathrm{PBT.}$

The incubation with the primary antibody was done over night on a wheel at $4^{\circ} \mathrm{C}$ diluted 1:100 in 3\% BSA. After incubation embryos were rinsed two times with PBT followed by two additional washing steps for 30 minutes on the wheel. After adding the secondary antibody (anti-rabbit coupled with Alexa Fluor 488; 1:1000) embryos were protected from light during all following incubation and washing steps. Incubation was performed for at least 90 minutes on wheel and then rinsed for two times with PBT. Staining of nuclei was performed with Hoechst.

\subsubsection{Edu proliferation assay and analysis}

To visualize cell proliferation in developing embryos a Click-iT ${ }^{\circledR}$ EdU (5-ethynyl-2 deoxyuridine) assay $\left(\right.$ Click-iT $^{\circledR}$ EdU Alexa Fluor ${ }^{\circledR} 488$ Imaging kit, Life technologies, Eugene OR, USA) was used (Salic and Mitchison, 2008). Egg collections of 0-1 h old embryos were kept for $16 \mathrm{~h}$ at $32^{\circ} \mathrm{C}$. Embryos were dechorionated two times in $1 \%$ commercial bleach for 40 seconds and placed on a microscope slide as described previously (Berghammer et al., 2009). Injection was performed using an end concentration of $50 \mu \mathrm{M} / \mu 1$ EdU dissolved in injection buffer. The injection procedure was done for a maximum of 1 hour at room temperature to keep the development of the embryos to a minimum. EdU was injected at the anterior part of the egg directly into the yolk. After each injection the microscope slides were placed on apple juice agar plates to avoid drying out of the embryos and incubated for three hours at $32^{\circ} \mathrm{C}$ to allow for proliferation. Afterwards embryos were dechorionated with $50 \%$ 
commercial bleach and washed on the slides using a pipette and a piece of paper tissue to carefully remove bleach and water without touching the embryos. Embryos were carefully transferred into scintillation vial with a paintbrush and fixed as usual. After fixation eggs still floating in the interphase were collected, washed with methanol for several times and transferred into PBT to allow dissection with beveled glass capillaries to release germ bands from their vitelline membrane. Staining of EdU treated embryos was done according to the ISH protocol (Schinko et al., 2009) and the manufacturer's instructions as described below. Before staining, embryos were rehydrated in 2:1 MeOH/PBT and 1:2 MeOH/PBT and then washed two times in PBT. Afterwards they were post fixed for 15 minutes in $1 \mathrm{ml}$ PBT and $140 \mu 1$ Formaldehyde (37\%). After washing with PBT, embryos were digested with $8 \mu \mathrm{g}$ Proteinase $\mathrm{K}$ for a maximum of 6 minutes and again post fixed, followed by two additional washing steps in PBT. After washing in BBT, embryos were incubated in 0,5\% TritonX100 for 20 minutes followed by two washing steps with BBT (last one for 20 minutes). The ClickiT® reaction cocktail was prepared according to manufacturer instructions shortly before using $500 \mu \mathrm{l}$ for approximately $30 \mu \mathrm{l}$ of embryos. After addition of the reaction cocktail embryos were protected from light and incubated for at least 30 minutes, followed by at least two washing steps in PBT. Afterwards, nuclei were stained for 10 minutes with $0,6 \mathrm{ng} / \mu \mathrm{l}$ Hoechst 33342 in PBT and washed for additional three times with PBT.

\subsubsection{Embedding}

After staining, germ bands of the embryos were transferred to $100 \%$ glycerol to dissect remaining yolk with an eyelash glued to a pipette tip. Whole mount in situ hybridization (ISH), EdU proliferation assay and cleaved Dcp-1 stained embryos were mounted in $100 \%$ glycerol. FM® 1-43 stained embryos were also mounted in $100 \%$ glycerol, but in order to avoid the squeezing of germ bands small pieces of plasticine were added to the cover slips as spacers.

\subsubsection{Cuticle preparations}

After egg collection, embryos were stored on small sieves (mesh size $300 \mu \mathrm{m}$ ) for additional 4 days at $32^{\circ} \mathrm{C}$. Eggs and first instar larvae were dechorionated in $50 \%$ commercial bleach. Afterwards they were embedded in 1:1 of Hoyer's medium/ lactic acid and incubated at $65^{\circ} \mathrm{C}$ overnight as described previously (Bucher and Klingler, 2004). 


\subsubsection{Statistical analysis}

Cell counting analysis was done using whole stacks of EdU treated wild-type and RNAi embryos. Cell counting of embryo stacks was done using Image J (Schneider et al., 2012). Each stack was divided into specific counting regions including the upper half of the headlobes as control, the labrum anlagen, as well as the first outgrowing leg pair (fig). The number of proliferating cells in the defined areas was counted with the "cell counter" plug-in from Image J. Statistical analysis of counted cells was performed using the statistics program $\mathrm{R}$ (http://www.r-project.org/) in combination with the $\mathrm{R}$ commander plug-in (RcmdrPlugin). To estimate the homogeneity of variances between counted cells both boxplot analysis and Saphiro Wilk test were performed (Shapiro and Wilk, 1965). Normal distribution was tested between both sides of one embryo. Since no significant difference between both sides was detected (see Figure 7.1), proliferating cells of each side were summed and the mean values generated. Subsequent tests were performed with this mean values. Normal distribution and significance was tested for proliferating cells in wild type against Tc-mibl RNAi embryos of all counted embryos. For samples, where normal distribution was reached, a two sample t- test was done. Samples showing no normal distribution were analyzed with the Welch t-test.

\subsubsection{Microscopy and imaging}

Phenotype screening of whole cuticles was done with a Zeiss Axioplan 2 Microscope using dark field. To analyze head bristle pattern as described (Schinko et al., 2008) the DIC filter set was used. Images of cuticles were taken using the FITC filter set with a mercury vapor lamp to visualize auto fluorescence and the ImageProPlus (Media Cybernetics, Rockville, USA) software. For z-projections of the cuticle stacks about 10-30 planes were recorded and deconvoluted using the "No Neighbour" method of ImageProPlus. For generation of one image "Maximum Projection" or "Sum Slices" of ImageJ was used. Imaging of embryos stained with NBT/ BCIP was done with the DIC filter sets. To detect the fluorescence of the Hoechst and TSA staining either DAPI or Cy3 filter sets were used. EdU incorporation was visualized using the yfp filter sets. For z-projections of the embryo stacks about 20-30 planes were recorded and deconvoluted using the "No Neighbour" method of ImageProPlus. For cell counting analysis z-stacks were inverted and levels adjusted using Image J. For visualization of dying cells with cleaved Drosophila Dcp-1 antibody staining, the FITC filter set was used. 
Imaging of embryos stained with Hoechst and FM® 1-43 was done with a Zeiss LSM780 using $405 \mathrm{~nm}$ for Hoechst and $550 \mathrm{~nm}$ lasers for $\mathrm{FM}{ }^{\circledR} 1-43$ stainings. Stacks of whole embryos were loaded into Amira v5.3.2. 3D models of the confocal stacks were rendered with the voltex module and default settings. All images were assembled and levels adjusted in Photoshop (versions CS2 or CS5). Labeling of figures and generation of schemes was performed in Photoshop (versions CS2 or CS5) or Inkscape. 


\section{Results}

\subsection{Selection of head phenotypes from the iBeetle screen}

In order to find yet unknown genes which could be potentially involved in the patterning of the most anterior part of the head, I used the iBeetle database with datasets of a large scale RNA interference (RNAi) screen in the red flour beetle Tribolium castaneum (Schmitt-Engel et al., accepted). I used data available on the iBeetle base after the first major screening phase of the iBeetle screen, where around 5,300 datasets were annotated. Each dataset included information related to phenotype induced by the injection of one individual iBeetle dsRNA fragment. This information comprised some experimental details, a detailed description of several types of phenotypes and related pictures. For my purpose the database was searched for cuticle phenotypes found in L1 larvae in the pupal injection screen (status 18.9.11). The data was searched broadly for head defects (examples in Figure 4.1) and the results were then further classified using all available data. Criteria were:

- Specific: Head phenotypes coupled with defects of abdominal segments were chosen only if the head phenotype was obvious.

- Interesting gene: phylogenetic analysis indicated to be a gene of interest because either function of the gene was not described in Drosophila so far or the gene was not implicated with head patterning processes before.

All in all 18 different phenotypes (see Table 4.1 and section 4.1.1) were selected for reinjection. 

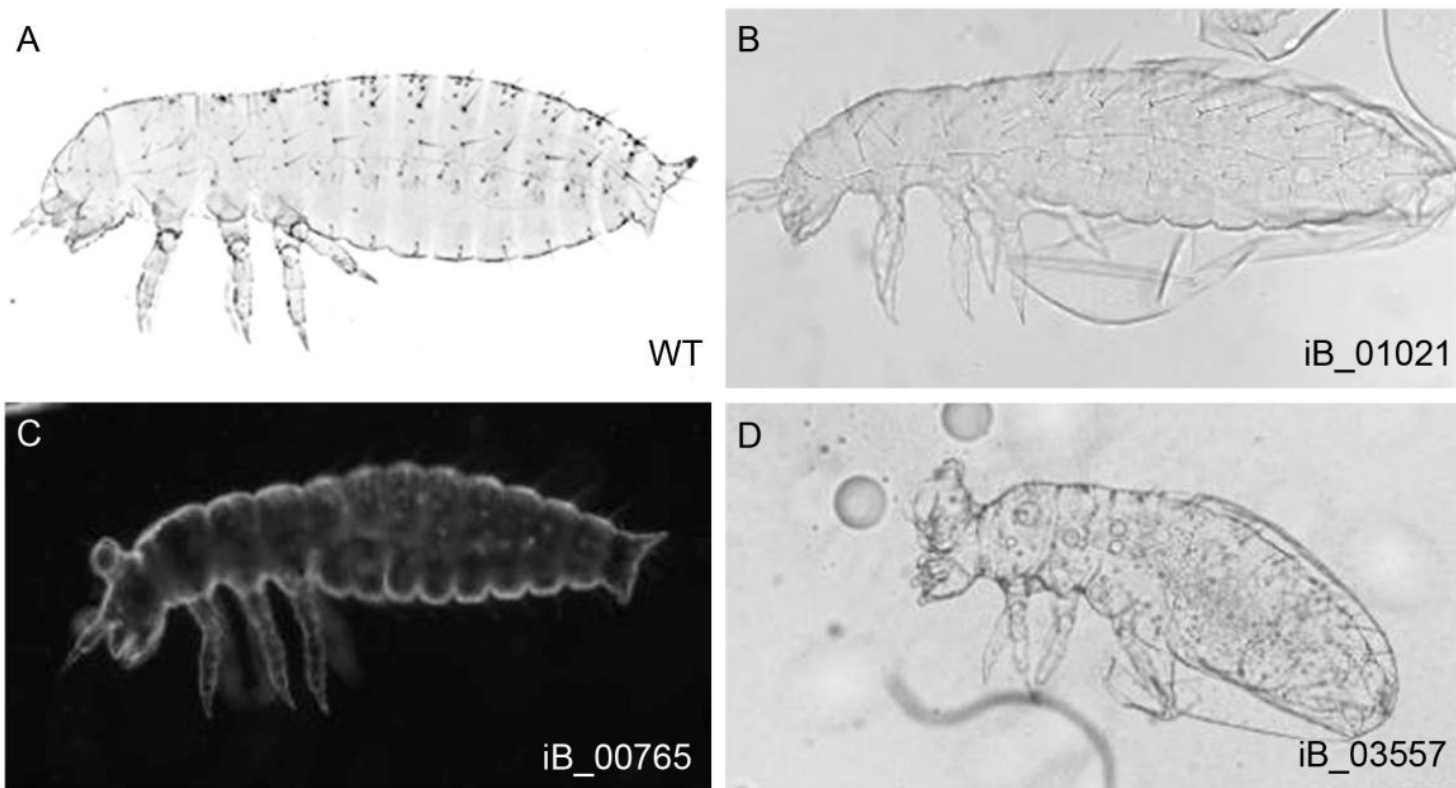

Figure 4.1: Examples of head phenotypes selected from the screen - Anterior is to the left. (A) Lateral view of a wild type cuticle. (B-D) Cuticle phenotypes. (B) Phenotype of iB_01021 resulted in a missing labrum. (C) RNAi of iB-00765 caused a displacement of the labrum and a reduction of the procephalic head. (D) Malformation of the procephalic head and protruding structures after knockdown of iB_03557.

\subsubsection{Rescreen and selection of final candidate genes}

It was recently shown that, some RNAi phenotypes depended on the genetic background of the injected strain (Kitzmann et al., 2013). In order to identify genes that produce similar phenotypes in different strains, dsRNA fragments of each gene were injected into the wild type strain San Bernadino. For iB_00765, iB_05247, iB_02692, iB_02582, iB_05264, iB_02268, iB_05634 and iB_03352 fragments were injected also into the strain Pig-19 which was already used in the screen. In addition, non-overlapping fragments of each gene were generated to check for possible off target effects. Moreover, gene knockdown was performed with two different concentrations $(1 \mu \mathrm{g}$ and $3 \mu \mathrm{g})$ to determine if the strongest phenotype was already described in the screen. The offspring of both strains was collected around 9 days after injection of pupae and cuticle analysis was done. Hatched L1 larvae were also analyzed for phenotypes. Based on these experiments 13 out of 18 genes were discarded due to different reasons (Table 4.1). The labrum phenotype of the genes iB_00765, iB_05247, iB_02582 and iB_01766 was reproduced with lower frequency but additional defects appeared like an enhanced amount of empty eggs (embryos which were not able to produce a cuticle at all) or unspecific disturbance of the abdominal segments. The annotated head phenotypes of the genes iB_02692 and iB_02350 were reproduced but additional unspecific 
defects were observed. For the genes iB_01725 and iB_04199 a part of head defects and defects of the abdominal segments were reproduced but not the labrum phenotype itself. The labrum phenotypes which showed only a penetrance under 30\% (iB_01021 and iB_01027) in the screen were not reproduced at all. Further, the head phenotypes of the genes iB_00951, iB_3557 and iB_00561 were not reproduced. In most cases, instead of the specific phenotype, phenotypes of the entire cuticle or a decreased egg production were observed as well as increased lethality. No obvious alteration was observed between the different dsRNA concentrations used in the injections.

\begin{tabular}{|c|c|c|c|c|}
\hline ID-number & Tc-number & Screen annotation & $\begin{array}{l}\text { Fragment/Strai } \\
n\end{array}$ & Reason to discard \\
\hline iB_00765 & TC_004781 & $\begin{array}{c}\text { Labrum lost }(30-50 \%) \text { or displaced } \\
(<30 \%) \text { head partly not present } \\
(<30 \%)\end{array}$ & $\begin{array}{c}\mathrm{NOF}+\mathrm{iB} / \mathrm{SB}+ \\
\text { Pig19 }\end{array}$ & \multirow{4}{*}{$\begin{array}{l}\text { Labrum phenotype } \\
\text { reproduced with lower } \\
\text { frequency but } \\
\text { additional unspecific } \\
\text { defects observed }\end{array}$} \\
\hline iB_05247 & TC012475 & $\begin{array}{c}\text { Labrum irregular }(<30 \%) \text { head shape } \\
\text { irregular }(50-80 \%) \text {, antenna } \\
\text { decreased }(<30 \%)\end{array}$ & $\begin{array}{l}\text { NOF / SB + } \\
\quad \text { Pig19 }\end{array}$ & \\
\hline iB_02582 & ТC016377 & $\begin{array}{c}\text { labrum irregular Procephalic head } \\
\text { lost }(<30 \%) \text {, head irregular }(50- \\
80 \%), \text { lr irregular } 30-80 \% \text {, lr split }> \\
30 \% \\
\end{array}$ & $\begin{array}{l}\text { NOF / SB + } \\
\quad \text { Pig19 }\end{array}$ & \\
\hline iB_01766 & TC010938 & labrum not present $(<30 \%)$ & NOF/SB & \\
\hline iB_01725 & TC010758 & $\begin{array}{l}\text { Labrum and antenna elongated (50- } \\
80 \% \text { ) head capsule decreased }(80 \%) \\
\text { maxilla increased }(50-80 \%)\end{array}$ & $\mathrm{NOF} / \mathrm{SB}$ & \multirow{4}{*}{$\begin{array}{l}\text { Labrum phenotype not } \\
\text { reproduced }\end{array}$} \\
\hline iB_04199 & TC006711 & $\begin{array}{c}\text { Labrum not present }(30-50 \%) \text {; head } \\
\text { capsule missing }(<30 \%) \text {, head } \\
\text { appendages randomly missing } \\
(<30 \%)\end{array}$ & NOF / SB & \\
\hline iB_01021 & TC006255 & Labrum not present $(<30 \%)$ & NOF / SB & \\
\hline iB_01027 & TC006291 & Labrum not present $(<30 \%)$ & NOF / SB & \\
\hline iB_02692 & TC003063 & Labium not present $(30-50 \%)$ & $\mathrm{NOF} / \mathrm{SB}+$ Pig19 & $\begin{array}{l}\text { Labium phenotype } \\
\text { reproduced but } \\
\text { additional unspecific } \\
\text { defects observed }\end{array}$ \\
\hline iB_02350 & TC014911 & $\begin{array}{l}\text { Head capsule dorsal decreased; head } \\
\text { missing }(<30 \%) \text {, many hatched }\end{array}$ & $\mathrm{NOF} / \mathrm{SB}$ & $\begin{array}{c}\text { Head defects but } \\
\text { additional unspecific } \\
\text { defects observed }\end{array}$ \\
\hline iB_00951 & TC005877 & $\begin{array}{l}\text { Head capsule missing less } 30 \% \\
\text { many hatched } 70-100 \%\end{array}$ & $\mathrm{NOF} / \mathrm{SB}$ & \multirow{3}{*}{$\begin{array}{l}\text { Head phenotype not } \\
\text { reproduced }\end{array}$} \\
\hline iB_03557 & TC003442 & $\begin{array}{l}\text { Procephalic head reduced }(50-80 \%) \\
\text { Head capsule reduced }(50-80 \%)\end{array}$ & $\mathrm{NOF} / \mathrm{SB}$ & \\
\hline iB_00561 & TC003368 & $\begin{array}{c}\text { Head capsule not present } 30-50 \% \text {, } \\
\text { head appendages randomly missing } \\
(30-50 \%)\end{array}$ & $\mathrm{NOF} / \mathrm{SB}$ & \\
\hline
\end{tabular}


Table 4.1: Genes discarded after re-injection - Given are iBeetle ID number (ID-number), the corresponding gene number found on the Tribolium gene browser (TC-number), the annotation of head defects from the screen (Screen annotation), injection of non-overlapping fragments (NOF) and original iB-fragments (iB) in $S B$ and/or pig-19 (Fragment/Strain) and the reason why this gene was no longer of interest (Reason to discard).

The phenotypes of four genes were reproduced. Both the iBeetle fragment and nonoverlapping fragment of iB_05264 (TC012539) led to a partial depletion of the vertex bristle pattern of the head capsule in both strains ( $S B$ and pig-19). Upon rescreen analysis the depletion was specified as the two setae marking the posterior and lateral vertex triplet setae (Figure 4.2B) Moreover, setae were not only depleted but sometimes duplicated and roundish cuticle structures remained at the location of the missing setae. Phylogenetic analysis revealed the gene Down syndrome cell adhesion molecule as closest fly homolog (Dscam). Nevertheless Tc-Dscam turned out to be mostly involved in sensory organ development but not in labrum formation. Therefore, this gene was not included in the final selection of candidate genes.

In addition, the phenotype of the iB_02268 (TC014275) fragment was successfully reproduced by non-overlapping fragments in the Pig-19 and the $S B$ strain. The ventral setae of the vertex triplet were irregular. Furthermore, the bristle pattern of the dorsal ridge was disturbed at the junction separating the posterior head from the trunk and dorsal closure of the trunk was affected (Figure 4.2C). Moreover, the urogomphi were affected (not shown). This gene was identified as an ortholog of the Drosophila gene kismet. Since the phenotype indicated an involvement in dorsal closure and thereby potentially in a more morphogenetic process, this gene was not included in the final selection of candidate genes. 


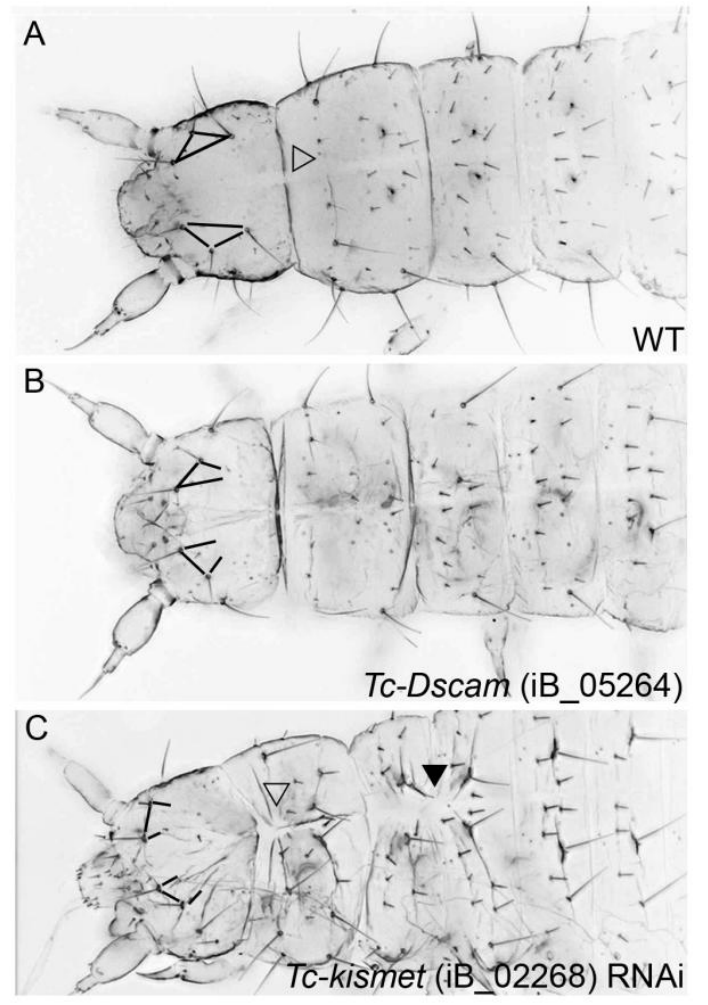

Figure 4.2: Phenotypes of iB_05264 and iB_02268 Cuticles in dorsal view. Anterior is towards the left. (A) Wild type cuticle marking the vertex triplet (black triangle). (B) Cuticle with iB_05264 phenotype. The most posterior setae of the vertex triplet was missing (compare triangle in $\mathrm{A}$ with open triangle in B). (C) Cuticle after iB_02268 knockdown. Mostly posterior setae of the vertex triplet were missing and the joint between head and thorax was irregular (compare region marked with open arrowhead in A and C). In addition, dorsal closure of the thorax was irregular (marked by black arrow in C).

Furthermore, the knockdown of the iBeetle fragment iB_03352 (TC002315) was annotated with a highly penetrant (over $80 \%$ ) head phenotype comprising a decreased head capsule with irregular head bristle pattern and particularly the lack of labrum tissue. In addition, also tracheal openings were lost or decreased. Upon rescreen analysis, the injection of one nonoverlapping fragment in the $S B$ and the Pig-19 strain resulted in a reproduction of all annotated phenotypes with similar penetrance. Phylogenetic analysis revealed the gene grain as closest fly homolog. This phenotype was further studied to some extent (see section 4.3 for detailed analysis).

The knockdown of the iBeetle fragment iB_05634 led to a highly penetrant labrum and leg phenotype (50-80\%). In the screen, dsRNA injection of this gene resulted in over $80 \%$ of the "L1 cuticle" in a complete loss of the labrum. The injection of two non-overlapping fragments resulted in a reproduction of all annotated phenotypes with similar penetrance in both strains (Pig-19 and SB). IB_04764 was annotated with a phenotype highly similar to the iB_05634 phenotype. Moreover, the annotated shortened leg phenotype (more than $80 \%$ ) was similar to the iB_05634 phenotype since structures of the leg were missing or fused. The gene with the ID number iB_05634 (TC014445) was identified as an ortholog of the Drosophila gene mind bombl and iB_04764 (TC010113) as an ortholog of the Drosophila gene Serrate. Injection of non-overlapping fragments in $S B$ was not performed since it was already published that $T c$ - 
Serrate plays of a role in metamorphic labrum, antenna and leg formation (Angelini et al., 2009; Angelini et al., 2012c; Smith et al., 2014). These phenotypes were studied in detail (see following sections).

\subsection{Notch signaling is involved in labrum formation}

\subsection{1 iB_05634 (Tc-mind bomb 1) and iB_04764 (Tc-Serrate) identified as genes both involved in the Notch signaling pathway}

AUGUSTUS gene predictions revealed that the dsRNA fragment with the iBeetle number iB_05634 targets a gene with the gene number TC014445. BLAST analysis of the protein sequence of this gene against non-redundant protein sequences of Drosophila melanogaster identified the gene mind bomb 1 as the closest fly homolog. (CG5841; Flybase ID FBgn0263601. The gene is also known as D-mib, l(3)72CDa, Dmib and Mib in Drosophila. The dsRNA fragment of iB_04764 targeted the gene with the number TC010113. The BLAST analysis of this gene revealed Serrate as the closest fly homolog. Interestingly, TcSerrate functions as a ligand of the Notch pathway directly interacting with mibl in Drosophila (Le Borgne et al., 2005b). In the following sections TC014445 and TC010113 will be referred as Tc-mind bombl (Tc-mibl) and Tc-Serrate (Tc-Ser), respectively. Phylogenetic analysis shows that Tc-Ser is the single ortholog of Dm-Ser while Tc-mibl is the single ortholog of Drosophila mibl.
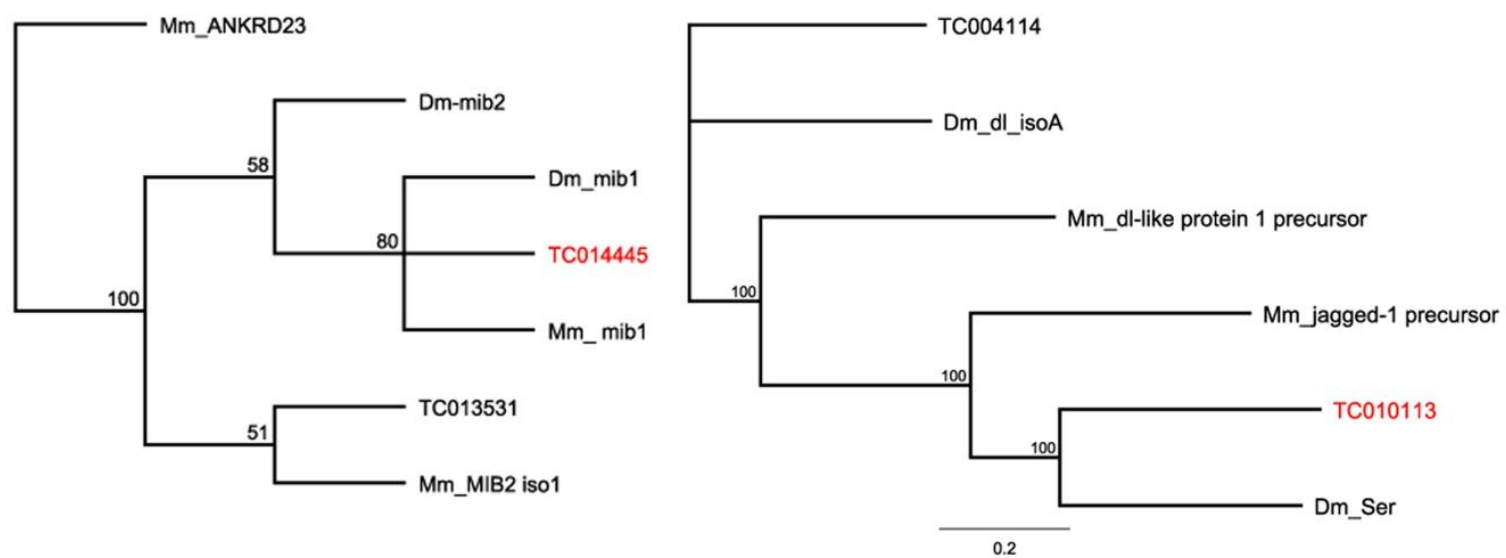

Figure 4.3: Phylogenetic analysis of iB_05634 (TC014445) and iB_04764 (TC010113) - Tree and image created with Geneious version 7.0. Phylogenetic analysis of the protein sequence showed that the gene TC014445 is the single ortholog to Dm-mib and Mm-mibl. Dm-mib2 is derived (A). Phylogenetic sequence analysis of TC010113 turned out to be the ortholog of Dm-ser and Mm-jagged. Dm= Drosophila melanogaster; 


\subsubsection{Functional analysis of $T c$-mind bomb1 and Tc-Serrate}

Quantitative analysis of 50 cuticles of both Tc-mibl and Tc-Ser knockdowns in the strain Pig19 revealed in a labrum phenotype with $100 \%$ penetrance. Mostly, the entire labrum was missing (compare arrowheads in Figure 4.4) including all four labrum quartet setae (for names of head setae see (Schinko et al., 2008)). The labrum was more frequently deleted (strong) in Tc-Ser RNAi animals because in a few cuticles of Tc-mibl RNAi animals the labrum was only reduced (weak) (Table 4.2). In addition, both knockdowns resulted in a shortened leg phenotype with $100 \%$ penetrance, with fused femur and tibiotarsus and a missing trochanter (Figure $4.4 \mathrm{~B}^{2}$ and $\mathrm{C}^{2}$ ). The leg phenotype after Tc-Ser knockdown was less strong. $42 \%$ of the analyzed cuticles showed a malformation comparable to Tc-mibl RNAi individuals (Table 4.2). Remnants of the trochanter and less shortening (weak) of the legs were observed in $58 \%$ of the analyzed Tc-Ser RNAi cuticles. Both RNAi treatments led to a malformed antenna. Mostly, one antenna was bent due to a malformation of the junction between antennifer and scapus (open arrowheads in Figure $4.4 \mathrm{~B}^{3}$ and $\mathrm{C}^{3}$ ). Again the antenna phenotype in Tc-mibl RNAi cuticles was more penetrant (Table 4.2). Both cuticle phenotypes also showed an irregular abdominal bristle pattern in some animals. In contrast to Tc-mibl RNAi, Tc-Ser RNAi resulted in an irregular maxillary palp surface at low frequency. Moreover, cuticles after $T c-S e r$ RNAi led to reduction or loss of tracheal openings with high frequency.

\begin{tabular}{|c|c|c|}
\hline Phenotype & $\begin{array}{c}\text { Tc-mibl RNAi } \\
\text { (penetrance in \%) }\end{array}$ & $\begin{array}{c}T c-\text { Ser RNAi } \\
\text { (penetrance in \%) }\end{array}$ \\
\hline Labrum affected & 100 (84 strong; 16 weak) & 100 (96 strong, 4 weak) \\
\hline Antennal malformation & 82 & 30 \\
\hline Legs shortened & 100 & 100 (42 strong; 58 weak) \\
\hline Abd. bristle pattern disturbed & 22 & 4 \\
\hline Maxilla abnormal & - & 30 \\
\hline Tracheal openings lost/decreased & - & 98 \\
\hline
\end{tabular}

Table 4.2: Quantitative analysis of Tc-mib1 and Tc-Ser RNAi phenotypes - Quantitative analysis of 50 cuticles. The penetrance of each phenotype is given in percent. Tc-mibl and Tc-Ser RNAi showed a similar phenotype with comparable penetrances. Tc-Ser resulted in additional defects of the maxilla and tracheal openings. Abd= Abdominal. 

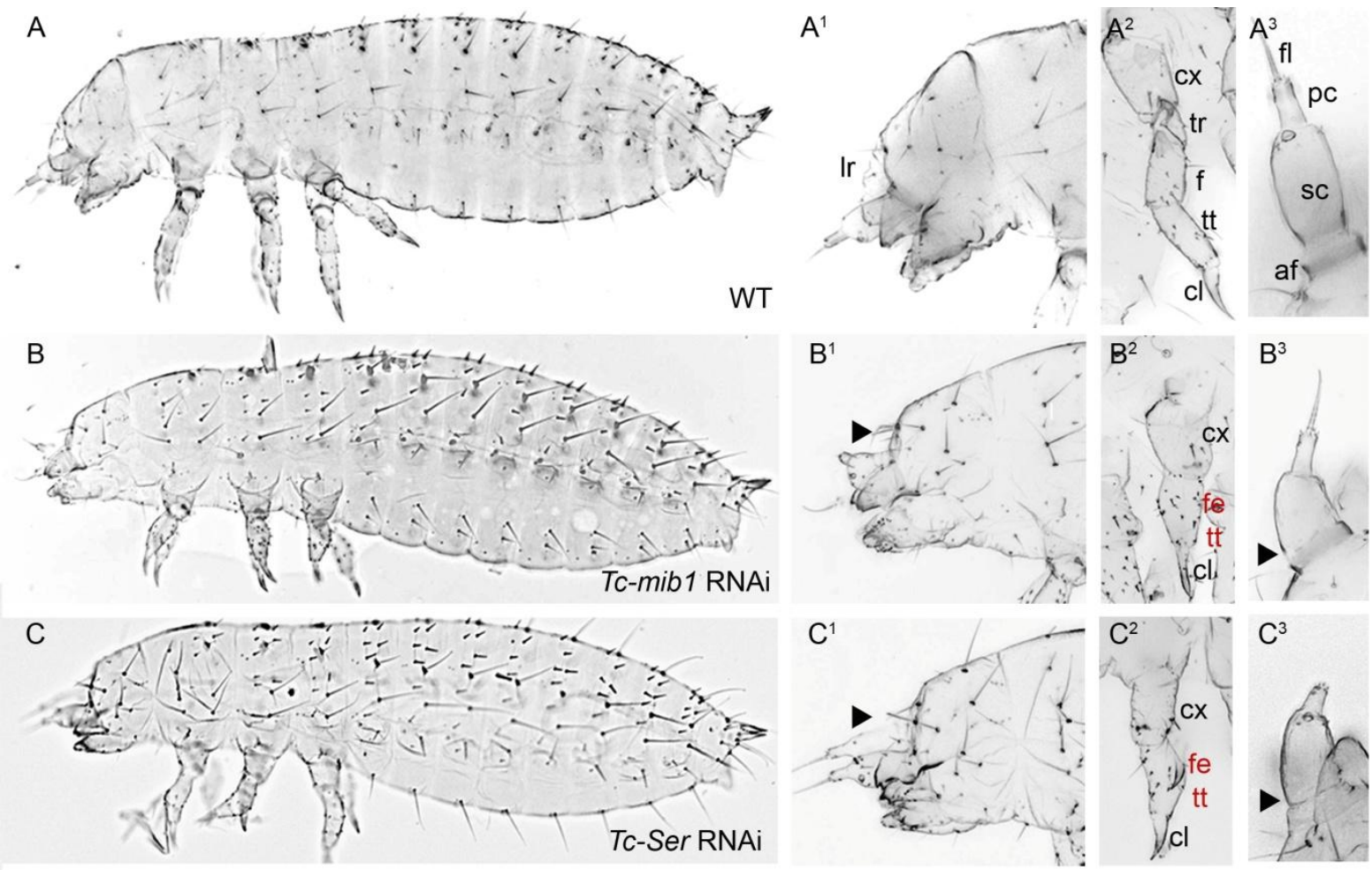

Figure 4.4: Cuticle phenotypes of $\boldsymbol{T} \boldsymbol{c}$-mibl and $\boldsymbol{T} \boldsymbol{c}$-Ser $\mathrm{RNAi}-\left(\mathrm{A}-\mathrm{A}^{3}\right)$ Wild type cuticles $\left({ }^{1}-{ }^{3}\right.$ represent higher magnification of the cuticle of head, leg and antenna). (B-B $\left.{ }^{3}\right)$ cuticles after Tc-mibl RNAi. (C-C ${ }^{3}$ ) Cuticles after Tc-Ser knockdown. Anterior is to the left. $\left(\mathrm{A}^{1}\right)$ In wild type cuticles the labrum (lr) was located between the antennae. $\left(\mathrm{A}^{2}\right)$ The leg is subdivided in five segments including coxa (cx), trochanter (tr), femur (fe), tibiotarus (tt) and claw (cl) (Grossmann et al., 2009). ( $\left.\mathrm{A}^{3}\right)$ The antenna consists of the antennifer (af), scapus (sc), pedicellus (pc), and flagellum (fl) (Toegel et al., 2008). After Tc-mibl and Tc-Ser RNAi the labrum was completely missing (compare arrowheads in $\mathrm{A}^{1}$ with $\mathrm{B}^{1}$ and $\mathrm{C}^{1}$ ). In addition, in both knockdowns a shortened leg phenotype was observed with a decreased or completely missing trochanter. Furthermore, the detected shortening of the legs was presumably enhanced to a fusion of femur and tibiotarsus (compare $\mathrm{A}^{2}$ with $\mathrm{B}^{2}$ and $\mathrm{C}^{2}$ ). In some RNAi cuticles, the antenna was bent and the junction between antennifer and scapus was slightly malformed (compare $\mathrm{A}^{3}$ with black arrowheads in $\mathrm{B}^{3}$ and $\mathrm{C}^{3}$ ).

In order to see when in embryogenesis the observed defects arise, and if RNAi embryos of $T c$ mibl and Tc-Ser resulted in different defects, embryos were stained for expression of the segment polarity gene $T c-w g$. RNAi of both genes resulted in loss of $T c-w g$ expression in the labrum anlagen indicating a role in early development. Since Tc-mibl was known to be required for activity of the Tc-Ser ligand (Le Borgne et al., 2005b) and no obvious difference was observed regarding the labrum phenotype, all subsequent experiments were done using the Tc-mibl knockdown. 


\subsubsection{Labrum development is initialized after disruption of Notch signaling pathway but fails to grow}

Previous investigations showed that the mutants of the gene Tc-Distal-less led to a complete loss of the labrum at cuticular level but that it remained visible as a reduced structure during embryogenesis (Beermann et al., 2001). Therefore, the embryonic phenotype of Tc-mibl RNAi was analyzed in detail. To estimate the stage at which labrum development is affected, RNAi embryos were stained with the membrane marker FM® 1-43. The morphology of these embryos was recorded with a confocal microscope and the resulting stacks were used for 3D reconstruction. During development the labrum starts to grow out as two buds (arrows in Figure 4.5A). Later during development, this two labral buds fused and formed a lobe like structure located between the antennae and anterior to the gnathal appendages (arrow in Figure 4.5B, E, $\mathrm{E}^{1}$ ) (Posnien et al., 2010). In contrast to the complete loss of the labrum observed in the first instar larval cuticle, the initial outgrowth of the labrum anlagen during embryogenesis was not disturbed since two distinguishable buds remained visible after $T c$ mibl RNAi (arrows in Figure 4.5C, D; F). However, a high reduction of the distal portion of the labrum was detected (compare arrows in Figure 4.5A with C). Later in development, the two labrum buds failed to fuse in RNAi embryos and two small bulb structures were still visible between the antennae (arrows in Figure 4.5D and $\mathrm{F}^{1}$ ). In addition, a view of a single slice in the middle of the embryo also showed the reduction of labrum tissue (compare arrows in Figure $4.5 \mathrm{E}^{2}$ with $\mathrm{F}^{2}$ ). Cuticle analysis of sibling embryos confirmed that strong phenotypes were analyzed. 
A

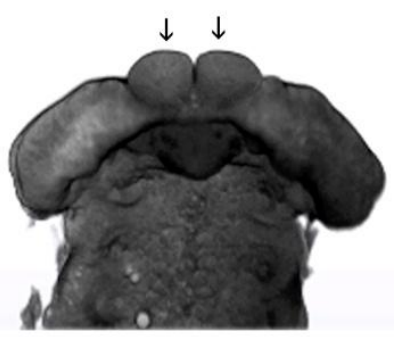

C

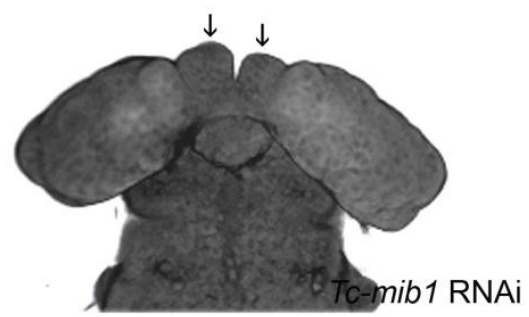

$\mathrm{E}$

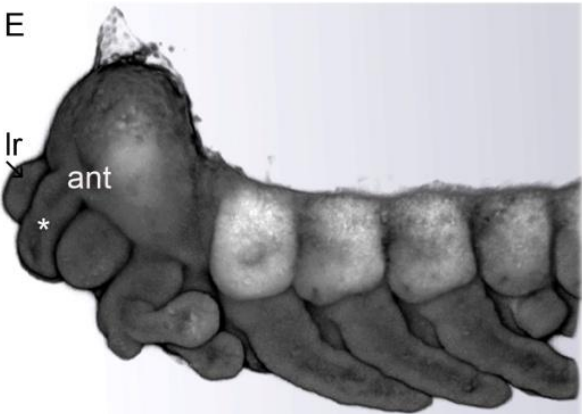

WT

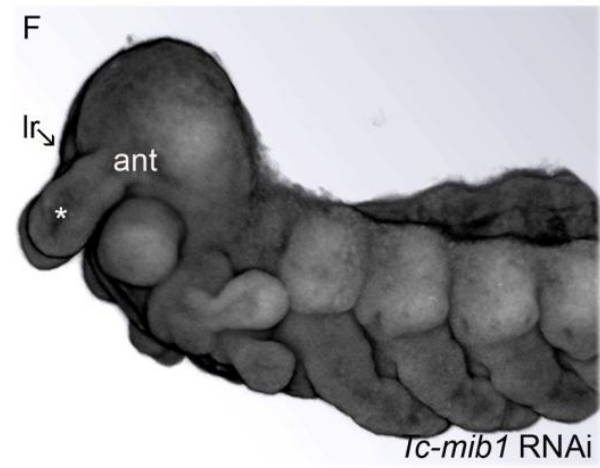

B

WT

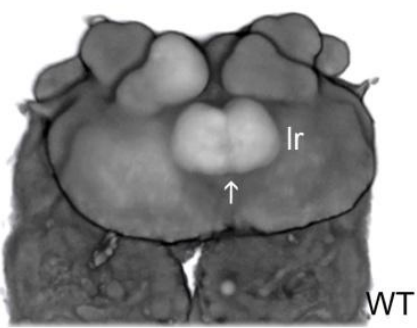

$\mathrm{D}$
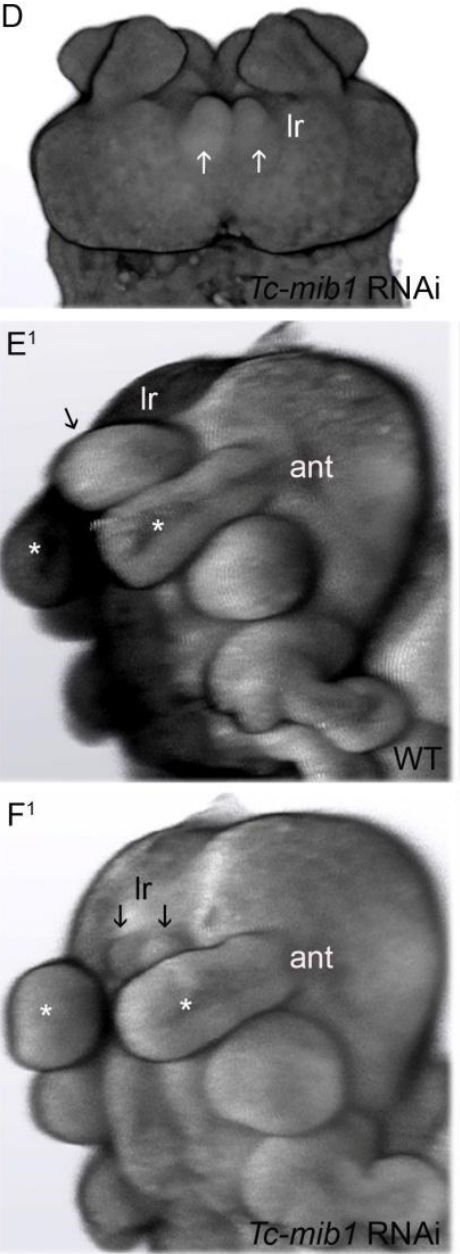
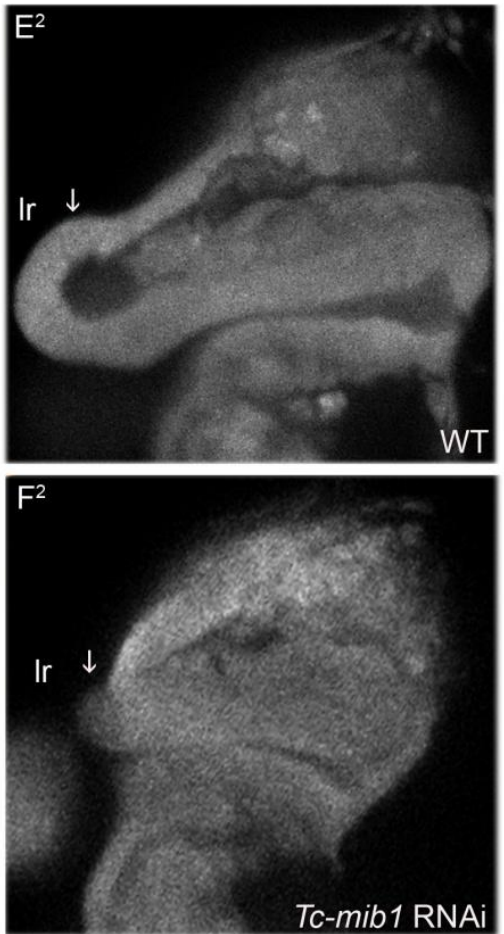

Figure 4.5: The labrum is reduced but not lost during embryogenesis after $T c-m i b 1$ RNAi - (A, B, E-E ${ }^{2}$ ) Wild type embryos. (C, D, F- $\mathrm{F}^{2}$ ) Tc-mibl RNAi embryos. $\left(\mathrm{A}-\mathrm{F}^{1}\right)$ 3D projections of stacks of FM® 1-43 stained embryos. $\left(\mathrm{E}^{2}\right.$ and $\left.\mathrm{F}^{2}\right)$ Detailed view of the head of the embryos seen on the left. Heads are shown in a different angle. ( $\mathrm{E}^{2}$ and $\mathrm{F}^{2}$ ) Single slices of the respective stack seen on the left; (A-D) Anterior is up; (E and F) anterior is left. (A) In wild type embryos the labrum (lr) is initialized as two separated labral buds (arrows). (B, E) Later in development the two labral buds fuse and are located between the antennae (ant, asterisk). (C) After knockdown of Tc-mibl the labral bulbs also start as two separated buds but were reduced in size (compare arrows in A, B with $\mathrm{C}, \mathrm{D})$. $\left(\mathrm{E}^{2}, \mathrm{~F}^{2}\right)$ The reduction after Tc-mibl RNAi was also clearly observed looking at the single slice in the middle of the embryonic head (compare arrows in $\mathrm{E}^{2}$ with $\left.\mathrm{F}^{2}\right) .\left(\mathrm{F}^{1}\right)$ The labrum anlagen remained as two small separated buds between the antennae in Tc-mibl RNAi embryos (compare arrows in $\mathrm{E}^{1}$ with $\mathrm{F}^{1}$ ). 


\subsubsection{Notch signaling components are active in developing labrum and leg anlagen}

In order to analyze the expression pattern of Tc- mibl during development in Tribolium in situ hybridisation with antisense and sense probes as negative control were performed. Tc- mibl was expressed ubiquitously during development. This was in accordance to the data available from $D$-mibl which was reported to be uniformly distributed in imaginal discs (Le Borgne et al., 2005b).
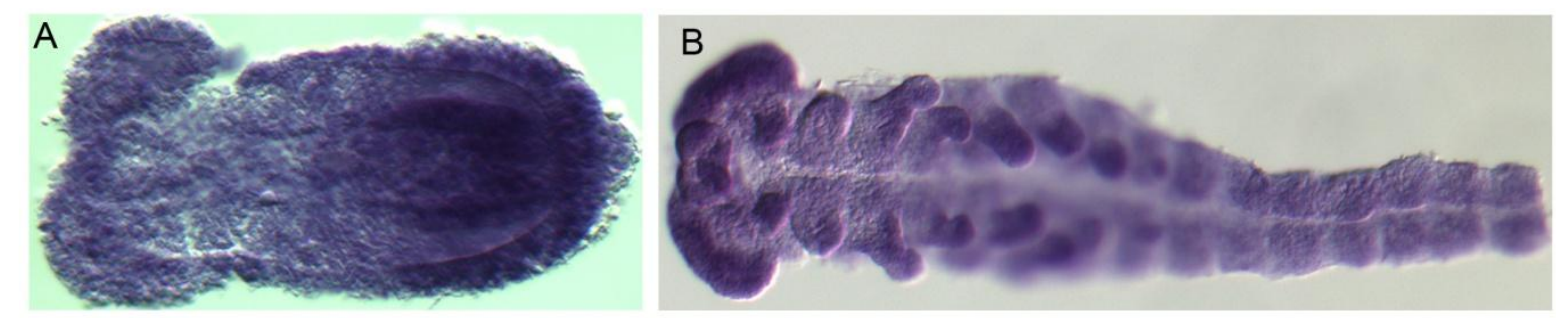

Figure 4.6: Expression of $\boldsymbol{T} \boldsymbol{c}$-mib1 - Anterior is to the left. Expression of $T c-m i b 1$ was expressed ubiquitously in germ rudiments and older elongating embryos.

Former investigations already demonstrated a localized expression pattern of $\mathrm{Tc}$-Ser in the outgrowing labrum anlagen, antenna and in the leg as ring shaped domains, at late elongation stages. So far the role of $T c$-Ser during embryogenesis was described in the context of Notch signaling analysis in Tc-hairy stripe formation in the growth zone (Aranda, 2006) and with respect to joint formation in the legs (Beermann et al., 2004). The phenotype analysis of TcSer revealed that the labrum was missing and legs shortened. Therefore, I determined the expression of $\mathrm{Tc}$-Ser at different developmental stages throughout development with focus on the labrum and leg domains. The first expression domain of Tc-Ser was observed already in early elongating stages as two lines along the anterior fold located in the anterior median region (AMR) (Kittelmann, 2012) (arrowheads in Figure 4.7 $\mathrm{A}^{1}$ and $\mathrm{B}^{1}$ ). This is in accordance with expression analysis of Serrate in Drosophila where expression also first occurred in the clypeolabrum (Fleming et al., 1990). During germ band elongation Tc-Ser expanded to form a broad domain marking the two labral buds which remained visible throughout development (arrowheads in Figure $4.7 \mathrm{C}^{1}-\mathrm{F}^{1}$ ). In addition, a second less intense domain arose in the head presumably marking cells of the nervous system (open arrowhead in Figure $4.7 \mathrm{C}^{1}$ ). Later a ring domain appeared in the outgrowing legs (asterisk in Figure $4.7 \mathrm{D}^{2}$ ) as well as expression started in the antenna and gnathal appendages (arrows in Figure 4.7D). During leg growth four additional rings of expression were observed marking the positions of the future leg joints (compare Figure $4.7 \mathrm{E}^{2}$ and $\mathrm{F}^{2}$ ) (Beermann et al., 2004). 

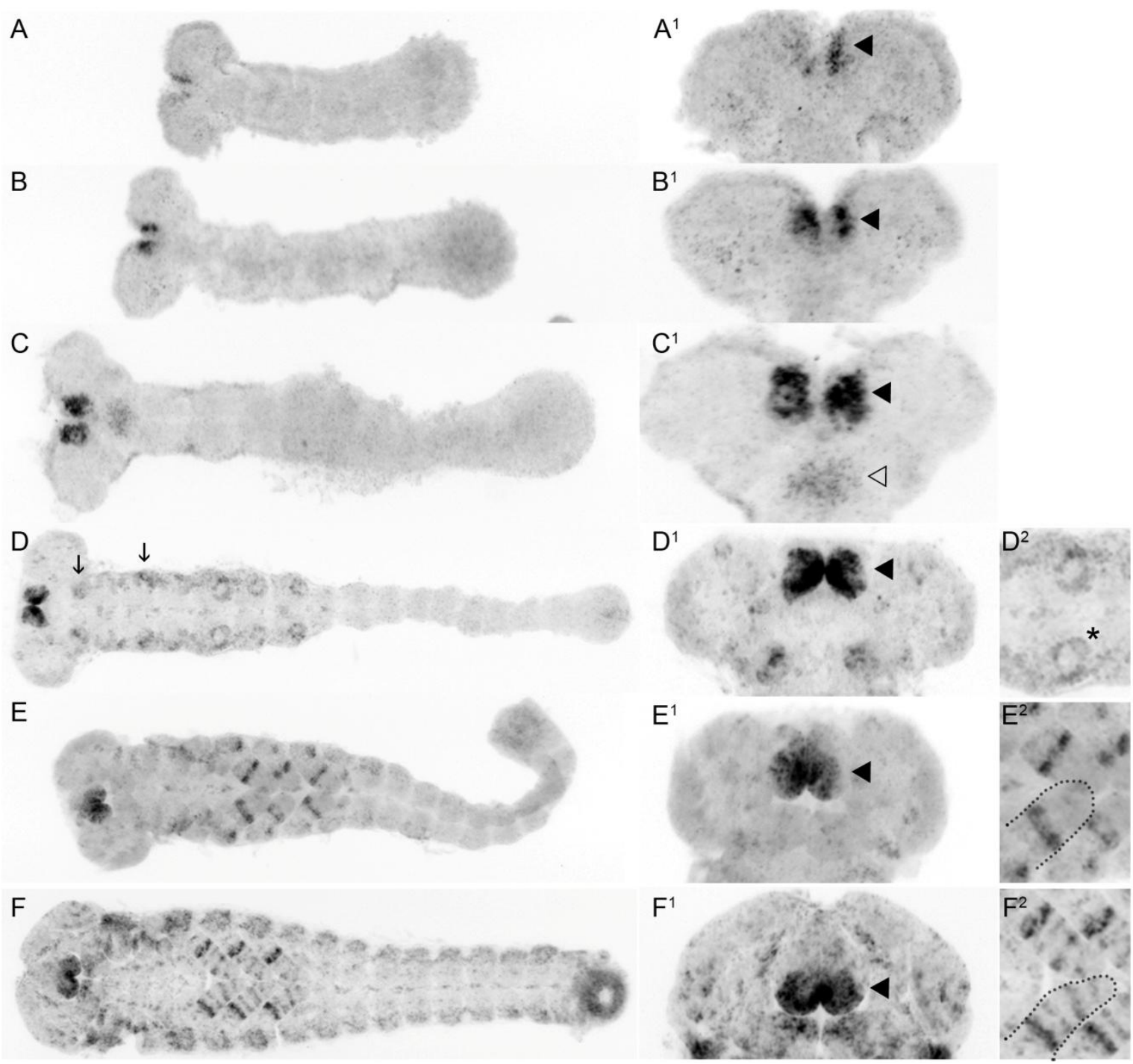

Figure 4.7: Tc-Serrate expression in Tribolium during development - (A-F) Embryos are oriented anterior to the left. $\left(\mathrm{A}^{1}-\mathrm{F}^{1}\right.$ and $\left.\mathrm{D}^{2}-\mathrm{F}^{2}\right)$ Detailed view of head and legs of the embryos seen on the left anterior to the top. (A,B) The expression of Tc-Ser started during early germ band elongation in the AMR (arrowheads in $\mathrm{A}^{1}, \mathrm{~B}^{1}$ ). (C-F) As elongation proceeded, this domain expanded to a broad domain in the growing labrum anlagen $\left(\mathrm{C}^{1}\right.$ $\mathrm{F}^{1}$ ). (C) Additionally, a domain arose presumably marking cells of the nervous system (open arrowhead inC $\mathrm{C}^{1}$ ). (D-F) After outgrow was initialized expression was also found in the appendages (arrows in D) and in a ring shaped domain in the leg anlagen (asterisk in $\mathrm{D}^{2}$ and $\mathrm{E}^{2}$ ). (F) In elongated legs five distinguishable ring domains were observed $\left(\mathrm{F}^{2}\right)$. Legs in $\mathrm{E}^{2}$ and $\mathrm{F}^{2}$ are marked with dotted lines.

\subsubsection{Notch signaling is not acting upstream of the AMR network}

The labrum develops as part of the anterior median region (AMR) (Hunnekuhl and Akam, 2014; Kittelmann et al., 2013). In order to test a possible role of Notch signaling in the gene regulatory network of the AMR, staining of genes which are expressed in this region and the surrounding tissue of different stages of development was performed after Tc-mibl RNAi. Expression pattern of $T c$-cnc; $T c-c r o c, T c-c h x$ and $T c$-six 4 have been previously described in 
Tribolium (Economou and Telford, 2009; Koniszewski, 2011; Posnien et al., 2011a) and will only briefly be mentioned with focus of the expression domains in the head. During early elongation, expression of $T c$-cnc seemed not to be affected after Tc-mibl knockdown, since two small domains remained expressed along the anterior fold of the embryo inside the AMR (compare Figure 4.8A with B). During development Tc-cnc expression expanded to a broad domain marking the labrum anlagen. After RNAi no clear difference could be detected in comparison to the wild type embryos (compare Figure $4.8 \mathrm{~A}^{1}$ with $\mathrm{B}^{1}$ ). Only at later stages, when labrum and appendages already started to grow out, the Tc-cnc labrum expression domain was reduced in sice of the tip of the labrum in RNAi embryos (compare arrowheads in Figure $4.8 \mathrm{~A}^{2}$ and $\mathrm{B}^{2}$ ). Nevertheless expression in the proximal part was not affected including the half ring around the posterior rim of the stomodaeum (asterisk in Figure $4.8 \mathrm{~A}^{2}$ and $\mathrm{B}^{2}$ ). Morphological analysis of the embryonic phenotype after loss of Tc-mibl function revealed that the labrum is initially formed but reduced in the most distal part of the buds. Therefore, this slight alteration of Tc-cnc expression was likely due to the loss of the labrum structure rather than due to direct regulation by Notch signaling.

No alteration of Tc-croc expression was observed in Tc-mibl RNAi embryos throughout development. Both in wild type and in RNAi embryos a single domain of expression was detected in early germ rudiments (Figure 4.8C, D). Even the Tc-croc domain in the proximal labrum and the domain which arose in the ocular region were not affected (compare Figure $4.8 \mathrm{C}^{1-2}$ with $\mathrm{D}^{1-2}$ ). 


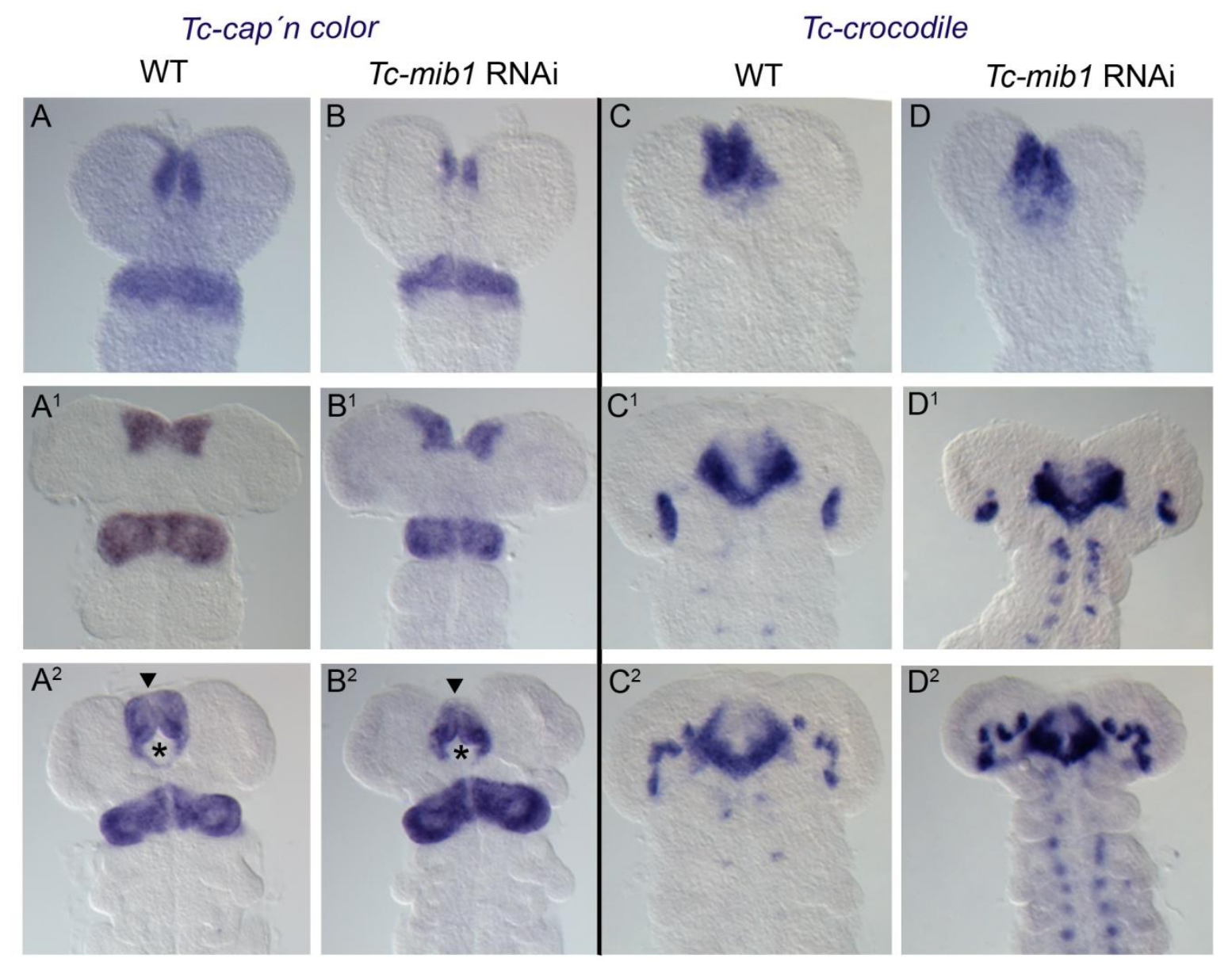

Figure 4.8: Early AMR patterning is not affected by $\boldsymbol{T}$-mib1 RNAi - All embryos anterior to the top. (A and C) Wild type embryos stained for $T c$ - $c n c$ and Tc-croc. (B and D) Corresponding expression in $T c-m i b 1$ RNAi embryos at approximately the same age. (A) During early elongation $T c$ - $c n c$ expression was observed in the $A M R$ and expanded later in the outgrowing labrum $\left(\mathrm{A}^{1}\right) .\left(\mathrm{A}^{2}\right)$ At later stages an additional line of expression was observed surrounding the stomodeum posterior (asterisk). (B-B ${ }^{1}$ ) After Tc-mibl RNAi these expression patterns were not affected during early elongating, but they were slightly reduced at later stages $\left(\mathrm{B}^{2}\right)$. The distal portion of labrum expression was reduced (see black arrowhead in $\mathrm{A}^{2}$ and $\mathrm{B}^{2}$ ) but unchanged in the proximal portion (asterisk in $\mathrm{A}^{2}$ and $\mathrm{B}^{2}$ ). (C) In wild type embryos Tc-croc expression started as a broad domain in germ rudiments. $\left(\mathrm{C}^{1}\right.$ and $\left.\mathrm{C}^{2}\right)$ During elongation the expression became restricted to the proximal part of the labrum and the stomodaeum. (D-D ${ }^{2}$ ) After knockdown no alteration was found (compare embryos in D with C).

$T c$-chx is expressed in a domain in the proximal part of the labrum and a stronger expression is marking neurogenic tissue lateral to the labrum (Figure 4.9A, C) (Koniszewski, 2011; Posnien et al., 2011b). In older elongating embryos an additional small domain in the ocular region was observed (arrowhead in Figure 4.9C). After Tc-mibl RNAi no alteration in size and intensity in all domains could be detected (compare Figure 4.9 A, C with B, D). However, the expression of the neurogenic tissue was slightly shifted (compare dotted lines in Figure 4.9C with D). As with Tc-cnc and Tc-croc, the difference between untreated and treated animals was more likely due to the reduction of the labrum after the knockdown. 
A similar effect was found for the expression domain of Tc-six4 in Tc-mibl RNAi embryos. In the head, Tc-six4 is expressed in the anterior rim region of the head lobes in wild type embryos (Figure 4.9E) (Posnien et al., 2011a). Tc-Six 4 expression after knockdown showed only a slight alteration of the orientation of the expression domain but no change in size and intensity (compare dotted lines in Figure 4.9C with D). This might be due to the loss of the distal portion of labrum tissue, which causes a slight movement of the surrounding head lobes.

In summary, the early aspects of expression of AMR marker genes were not affected. Later, slight alterations in the expression patterns in the AMR were observed. These alterations were changes in orientations of the expression domains rather than domain size or intensity. These results indicated that Notch signaling in the labrum is not involved in early AMR pattering.

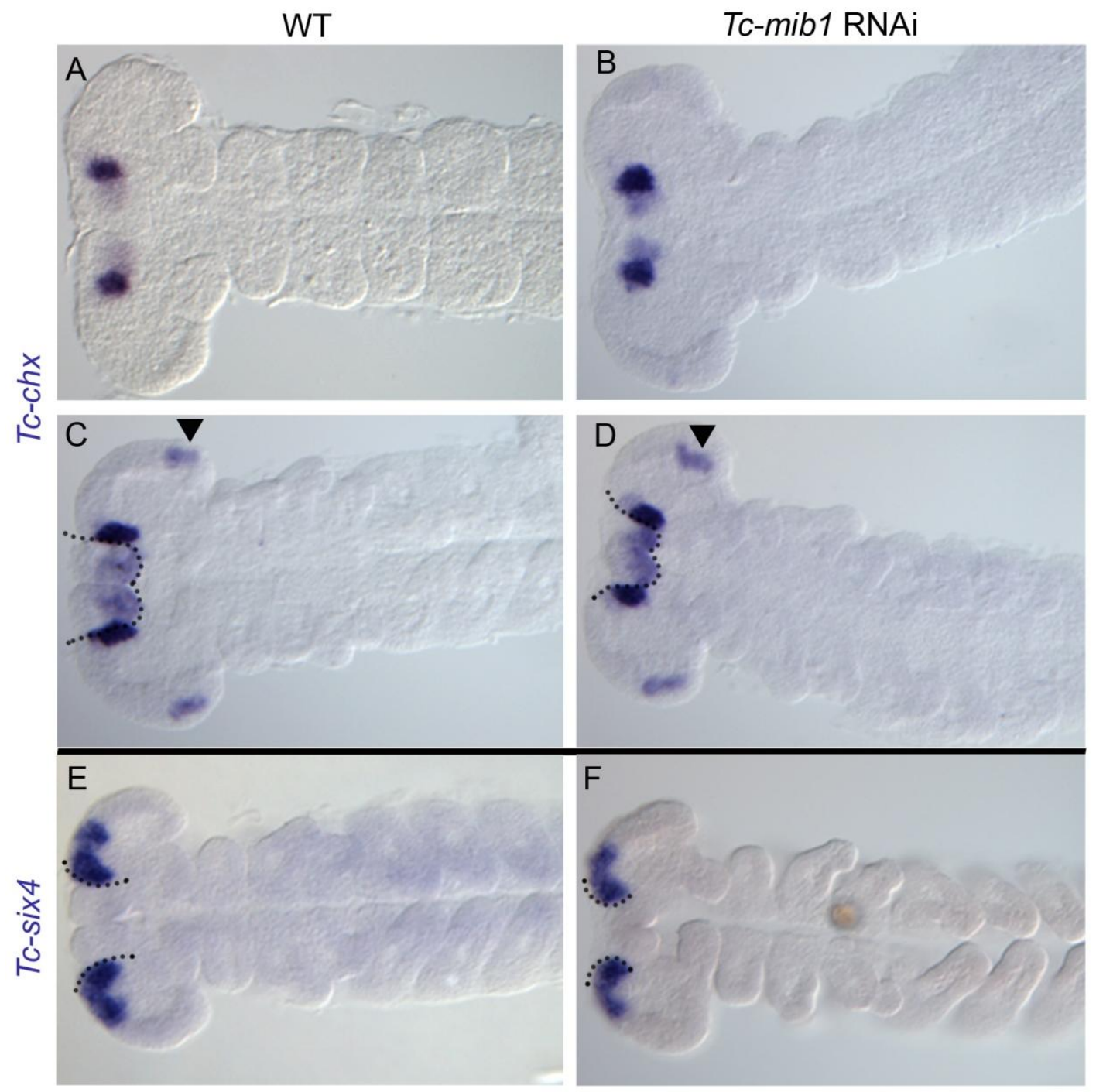

Figure 4.9: Mild shifts of expression domains of $\boldsymbol{T c}$-chx and $\boldsymbol{T} \boldsymbol{c}$-six4 $\mathbf{\text { in }} \boldsymbol{T} \boldsymbol{c}$-mib1 RNAi - Anterior is to the left. Embryos in one row are approximately of the same age. (A, C, E) wild type embryos. (B, D; F) RNAi embryos. (A-D) The expression of $T c$-chx was not affected after Tc-mibl RNAi regarding to the insensitivity of 
expression. Both domains in the labrum and the surrounding tissue were observed (compare A; C with B; D). (C, D) In older elongating embryos the expression of $T c$-chx marking the neurogenic tissue seems to be slightly shifted apart (compare marked region in $\mathrm{C}$ with D). (E, F) The intensity of Tc-six4 expression was also not affected after Tc-mibl RNAi but showed a shift inwards (compare angle of dotted lines in E with F).

\subsubsection{Role of Notch signaling in the appendage regulatory gene network}

It was shown previously that the genetic network of appendage and labrum patterning is probably conserved since expression domains arose in similar domains both in labrum and leg anlagen. However, loss of function analysis revealed a difference between appendages and the labrum. For instance, the labrum need neither Tc-hedgehog nor Tc-wingless signals to become initiated (Posnien et al., 2009a). In order to test to what extent the labrum malformation after disruption of Notch signaling was due to disturbance of the appendage regulatory network, expression dynamics of appendage marker genes in relation to Tc-Ser expression and their alteration after $T c-m i b 1$ RNAi was analyzed.

\subsubsection{Expression dynamics of proximal-distal patterning genes in labrum and legs}

Besides $T c$-Ser, expression in both labrum and trunk appendages has already been known for other proximal-distal patterning including the genes $T c-d p p, T c-w g, T c-D l l$ and $T c-d a c$. In order to analyse the dynamics of expression in the labrum was initiated in comparison to these genes staining was performed in embryos approximately at the same time point of development. The expression of $T c-d p p, T c-w g, T c-D l l$ and $T c-d a c$ was described in detail before (Beermann et al., 2001; Nagy and Carroll, 1994; Prpic et al., 2001; Sanchez-Salazar et al., 1996) and will only be mentioned in the analyzed context. Tc-Ser was the first gene to be expressed in the AMR shortly before expression of $T c-d p p$ and $T c-D l l$ was detectable (compare arrowheads in Figure 4.10A with $\mathrm{B}^{1}$ and $\mathrm{C}^{1}$ ). Later, during germ band elongation, two stripes of $T c$-wg expression were observed (arrowhead in Figure $4.10 \mathrm{D}^{2}$ ). When the legs started to grow out, $T c$-dac became expressed in the labrum (arrowhead in Figure 4.10E ${ }^{3}$ ). In addition, it became apparent that only $T c-S e r$ and $T c$-Dll were expressed inside the head before additional expression in the posterior part of the embryo was initiated (Figure 4.10A and $\mathrm{C}^{1}$ ). This order of expression indicated that $\mathrm{Tc}$-Ser may act upstream in labrum development compared to the other genes. In contrast to the early expression in the AMR, expression of $\mathrm{Tc}$-Ser in the trunk appendages started when the legs grow out (open arrowhead 
in Figure $4.10 \mathrm{~A}^{3}$ ). This was shortly after the expression domains of $T c-d p p, T c-D l l$ and $T c$ $d a c$ were already initialized in the trunk appendages (compare Figure $4.10 \mathrm{~A}^{2}$ with open arrowheads in $\mathrm{B}^{2}-\mathrm{D}^{2}$ ).
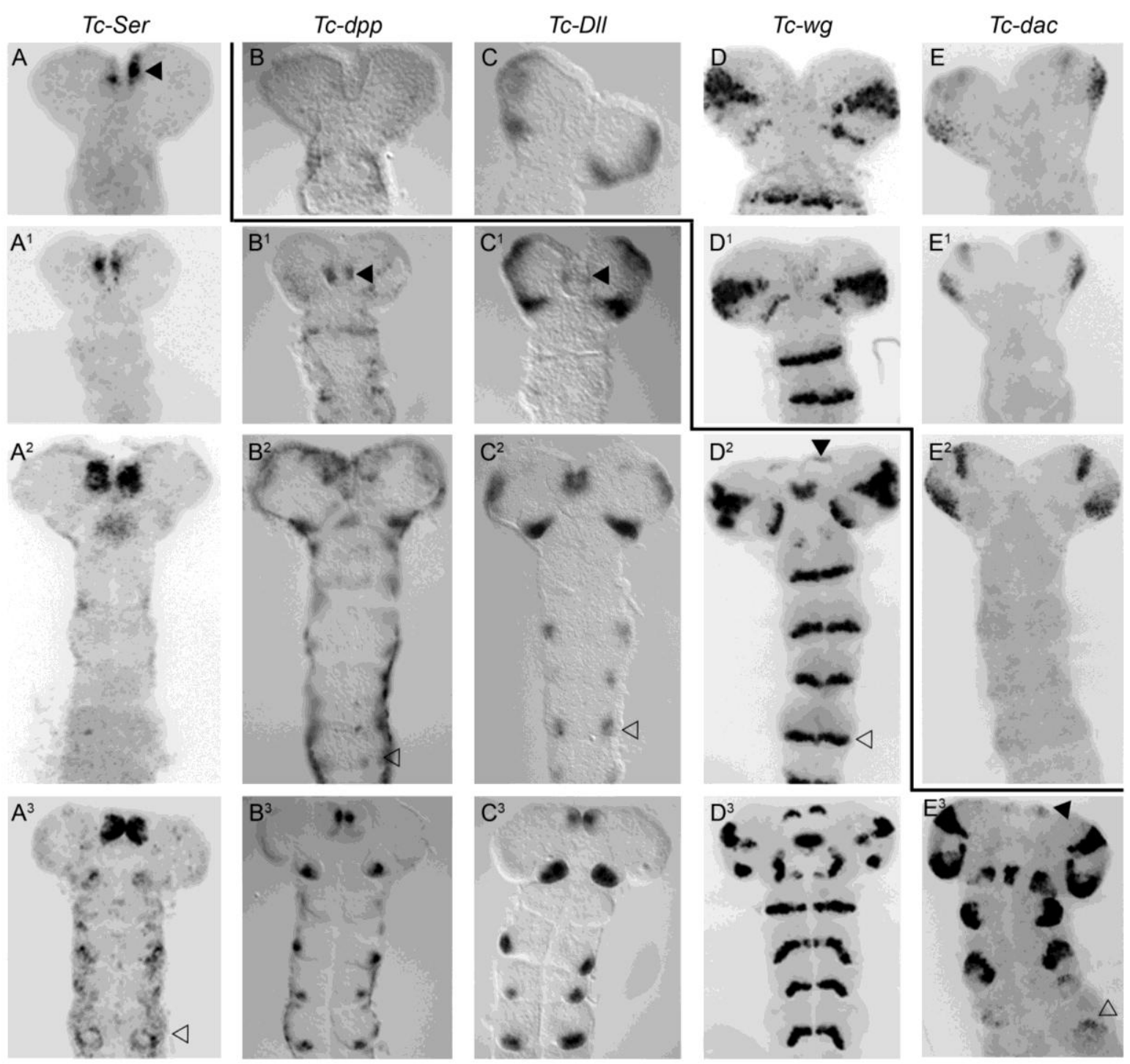

Figure 4.10: Initialization of labrum expression in proximal-distal patterning genes - Each column represents the expression domain of the same gene during development. Embryos in a row show approximately the same developmental time point. All embryos are oriented with the anterior to the top. (A, D and E) Embryos were stained with TSA reaction (Dylight 550). (B and C ) Embryos stained with NBT/BCIP reaction. $\left(\mathrm{A}-\mathrm{A}^{1}\right)$ Expression of $T c$-Ser was visible inside the AMR along the anterior fold in two separated domains marking the labrum anlagen during early germ band elongation. $\left(\mathrm{A}^{2}-\mathrm{A}^{3}\right)$ As development proceeded this expression expanded within the labral buds. $\left(\mathrm{B}^{1}, \mathrm{C}^{1}\right)$ Slightly later, during elongation also the expression of $T c$ $d p p$ and $T c$-dll started inside the AMR along the anterior fold (arrowheads in $\mathrm{B}^{1}$ and $\mathrm{C}^{1}$ ). $\left(\mathrm{D}^{2}\right)$ When appendage outgrow was initialized, $T c-w g$ expression was observed in a small domain marking the labrum anlagen. $\left(\mathrm{E}^{3}\right)$ Later, also $T c-d a c$ expression domains arise in the labrum as two weak spots in a dorso-lateral position. $\left(\mathrm{B}^{2}-\mathrm{D}^{2}\right)$ Expression domains of $T c-d p p, T c-D l l$ and $T c$-wg in the trunk appendages is initialized before leg appendages are morphological visible. $\left(\mathrm{A}^{3}\right.$ and $\mathrm{E}^{3}$ ) Shortly later, expression of $T c$-Ser and $T c$-dac is visible in the trunk appendages (open arrowheads). 
In order to determine the expression of $T c-S e r$ both in labrum and leg anlagen relative to the expression of the proximo-distal patterning genes, double stainings were performed. In the labrum the most distal part of $T c$-Ser expression probably overlapped the most proximal domains of $T c-d p p$ and $T c-D l l$ expression (Figure $4.11 \mathrm{~A}^{3}, \mathrm{~A}^{4}$ and $\mathrm{B}^{3}, \mathrm{~B}^{4}$ ). In contrast, no overlap was found in the outgrowing legs since $T c-D l l$ and $T c-d p p$ were separated from the more proximal ring domain of $T c$-Ser expression at this time of development where only one of the five ring domains was present (Figure $4.11 \mathrm{~A}^{3}, \mathrm{~A}^{5}$ and $\mathrm{B}^{3}, \mathrm{~B}^{5}$ ). In the labrum no overlap was observed regarding to $T c-w g$ expression (Figure $4.11 \mathrm{C}^{3}, \mathrm{C}^{4}$ ) but probably in the leg (Figure $4.11 \mathrm{C}^{3}, \mathrm{C}^{5}$ ). Tc-dac expression occurred as a half-circle in the distal portion of the labrum but due to its more lateral domain it did not overlap with Tc-Ser expression (Figure $4.11 \mathrm{D}^{3}, \mathrm{D}^{4}$ ) However, it did share a small region of expression in the leg in a weaker ring domain located proximal of the outgrowing limb (Figure $4.11 \mathrm{D}^{3}, \mathrm{D}^{5}$ ). 

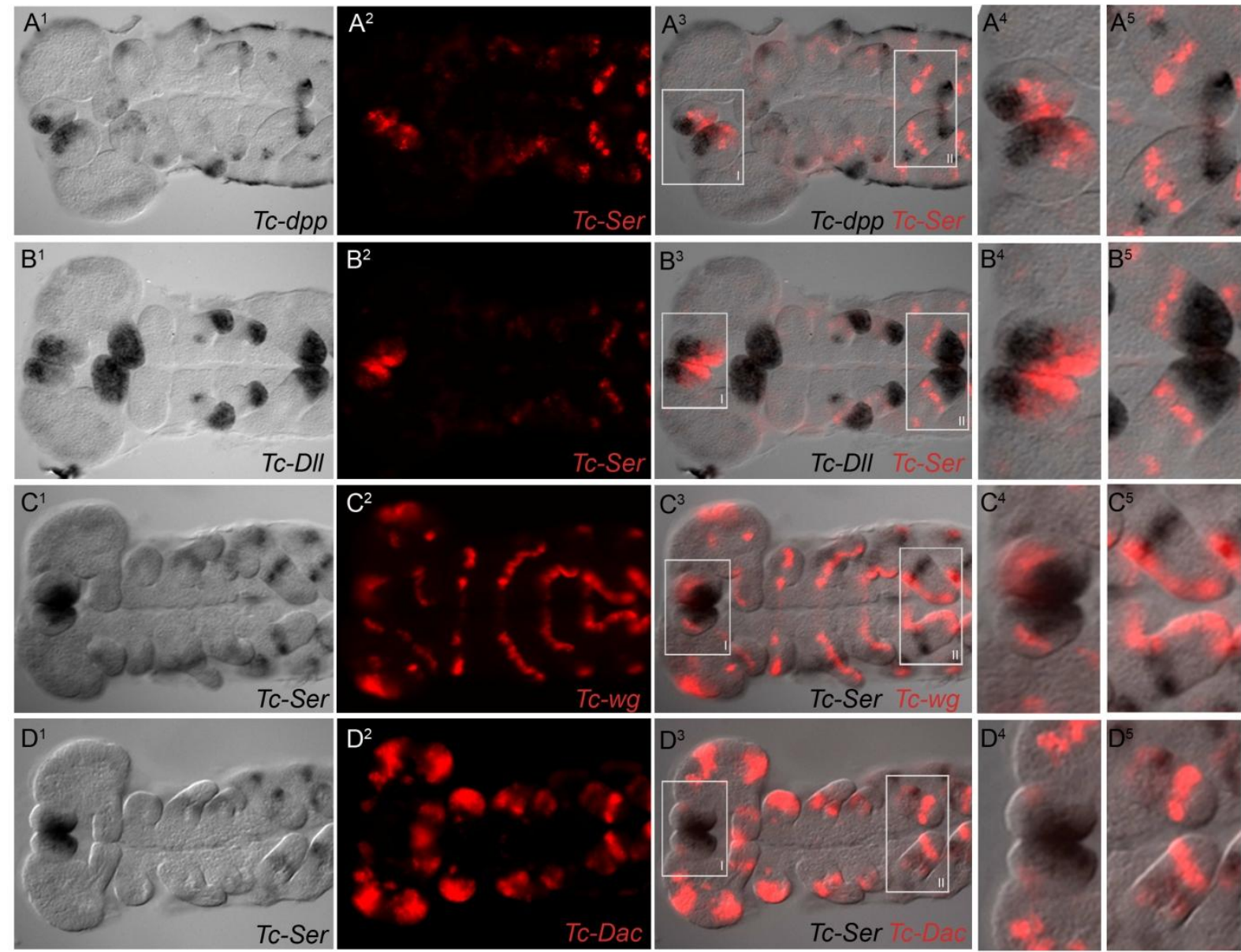

E trunk app.

labrum
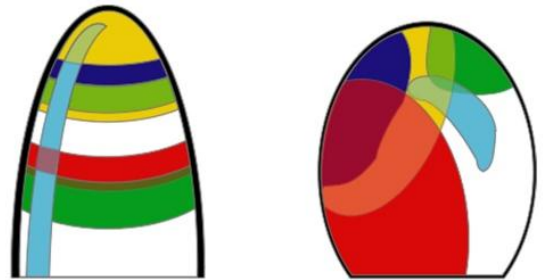

\section{TC-DII \\ $T c-d p p$}

Tc-dac

TC-wg

Figure 4.11: Double staining of $T c$-Ser with $T c-d p p, T c-D l l, T c$-wg and $T c$-dac - (A-D) Anterior is to the left. Staining of elongating embryos shortly after limb outgrow. $\left(A^{3}-D^{3}\right)$ overlay of embryos seen in $A^{1}-D^{1}$ and $A^{2}-D^{3}$. $\left(\mathrm{A}^{4}-\mathrm{D}^{4}, \mathrm{~A}^{5}-\mathrm{D}^{5}\right)$ Detailed view of marked regions seen in $\mathrm{A}^{3}-\mathrm{D}^{3}$. (A, B) $T c-d p p$ and $T c-d l l$ expression in black (NBT/BCIP); Tc-Ser in red (TSA-Dylight550). (C, D) Tc-Ser in black (NBT/BCIP); Tc-wg and Tc-dac expression in red (TSA-Dylight550). (E) Schema of proximal-distal patterning genes in the trunk appendages and the labrum. (A) In the labrum $T c-d p p$ expression overlaps in his most proximal part with $T c-\operatorname{Ser}\left(\mathrm{A}^{4}\right)$. No overlapping region was observed in the legs where $T c-d p p$ expression was localized much more distal $\left(\mathrm{A}^{5}\right)$. (B) The expression domain of $T c-D l l$ in the growing limbs was observed in the most distal portion. In the labrum it was presumably overlapping with the distal part of $T c$-Ser expression $\left(\mathrm{B}^{4}\right)$ but not in the legs $\left.\mathrm{B}^{5}\right) .\left(\mathrm{C}^{2}, \mathrm{C}^{3}\right) T c-w g$ was expressed in a proximal-distal stripe both in the labrum and the leg anlagen, but expression only overlapped with $T c$-Ser in the labrum $\left(\mathrm{C}^{4}\right) .\left(\mathrm{D}^{2}, \mathrm{D}^{3}\right) T c$-dac was detected in two separated ring domains. In the proximal part a weakly and in the distal part of the leg a more intense domain was observed. The Tc-Ser expression domain was located in between these two rings overlapping with the proximal one $\left(D^{5}\right)$. No overlap was observed in the labrum where $T c-d a c$ is expressed in a dorsal-lateral position $\left(\mathrm{D}^{4}\right)$. (E) In the outgrowing trunk appendages the expression Tc-Dll overlaps with $T c$-wg and the distal $T c$-dac expression (Posnien et al., 2009a; Prpic et al., 2001). In addition, $T c$-Ser presumably shared the expression domain with $T c-w g$ and the proximal $T c$-dac expression ring. In the labrum Tc-Dll overlaps with $T c$-wg, Tc-dac (Posnien et al., 2009a; Prpic et al., 2001) and Tc-Ser. Furthermore, Tc-Ser expression in the labrum overlaps with Tc-dpp. 


\subsubsection{Changes in expression after Tc-mib1 RNAi}

Knockdown of Tc-mibl and Tc-Ser RNAi resulted in a strong phenotype in the legs and the labrum. In addition, Tc-Ser expression was localized in these structures. In order to test whether both phenotypes originated due to a similar disturbance of the appendage regulatory network, the expression of the appendage marker genes in Tc-mibl RNAi embryos was investigated. Effects on $T c$-wg expression were investigated after Tc-mibl (Figure 4.12) and $T c$-Ser RNAi (not shown). In wild type embryos, $T c-w g$ expression was observed in a longitudinal stripe both during the outgrowth of the labrum and the legs (Figure 4.12A and C.) In Tc-mibl RNAi embryos the expression domain of $T c-w g$ in the labrum was lost (compare arrowheads in Figure 4.12A-D). In contrast, the expression domain in the leg anlagen showed no alteration between wild type and knockdown embryos (compare open arrowheads in Figure 16A-D). Likewise, after knockdown of Tc-Ser the same alteration of $T c-w g$ expression was observed in the labrum domain but not the legs (not shown). In wild type embryos a weak domain of $T c-d a c$ expression is observed in a half circle in the labrum shortly after outgrowth of the labral buds and a corresponding ring domain in the outgrowing legs (Figure 4.12E). Later, also a second ring domain of $T c-d a c$ expression was detected in the legs (Figure 4.12G). After Tc-mibl RNAi, the expression domain was deleted in the labrum anlagen similar to the loss of $T c$-wg expression (compare arrowheads in Figure 4.12 E-F). In the legs no alteration could be detected in both ring expression domains of $T c$-dac (compare open arrowheads in Figure 4.12E-F). 


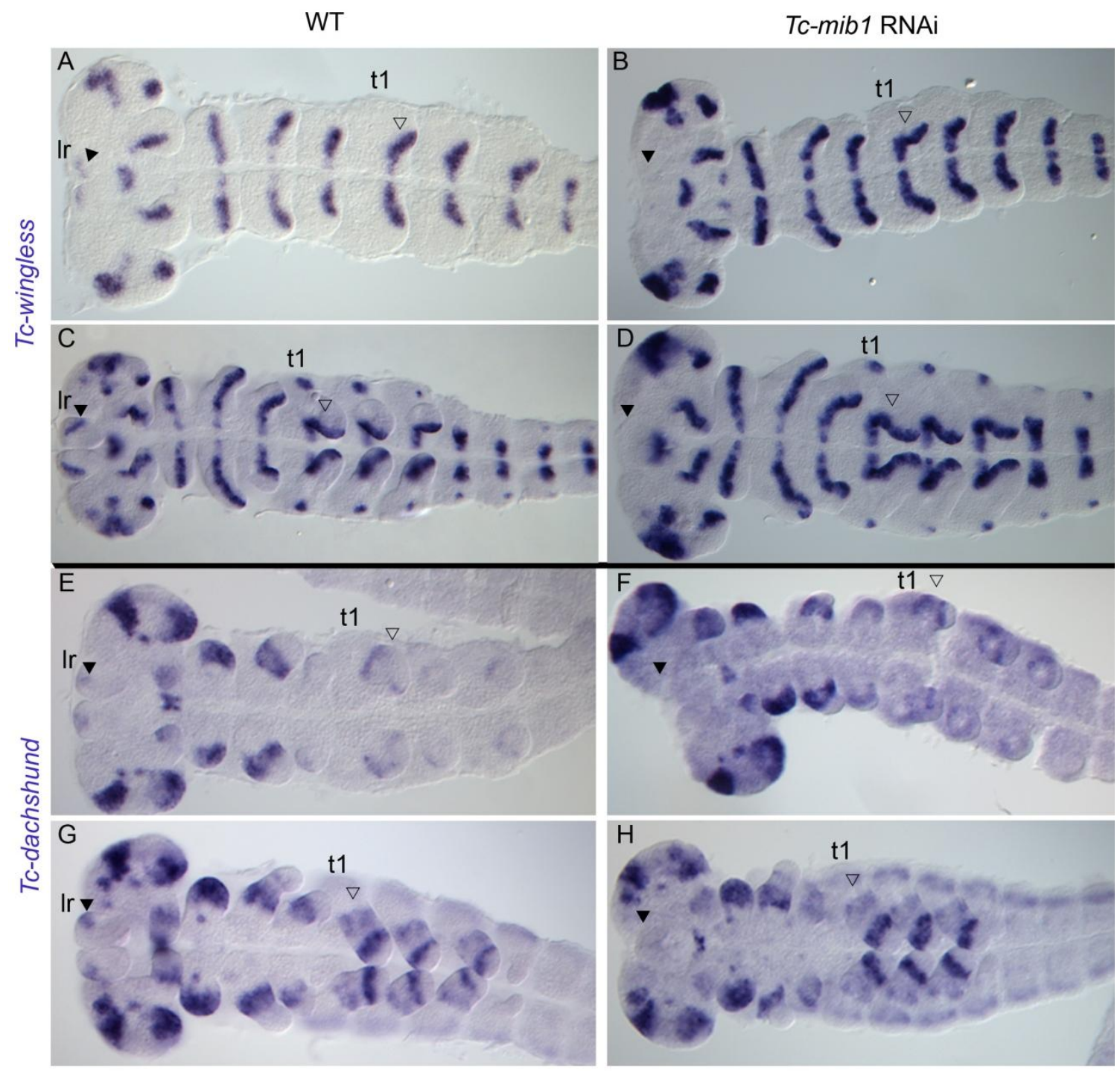

Figure 4.12: $T c$-mib1 RNAi affects expression of $T c-w g$ and $T c$-dac in the labrum - Anterior is to the left. Embryos in one column are of the same age. (A,C,E,G) Wild type embryos; (B; D; F, H) Embryos after Tcmibl knockdown. (B, D) Tc-wg expression was completely lost in the labrum after Tc-mibl RNAi (compare black arrowheads in $\mathrm{A}, \mathrm{C}$ with $\mathrm{B}, \mathrm{D})$. In the legs, the $T c$-wg expression was not altered (compare open arrowhead in A, C with B, D). (F, H) The expression domain of $T c$-dac was not detectable in the labrum after knockdown of Tc-mibl (compare arrowheads in E,G with F,H) but expression in the legs remained unaffected (compare open arrowheads in $\mathrm{E}, \mathrm{G}$ with $\mathrm{F}, \mathrm{H})$. (lr= labrum, $\mathrm{t} 1$ = throraric segment 1).

In early elongating embryos the expression of $T c-D l l$ starts in the procephalic head region, the antennal and maxillary segments. At the same time, expression also occurred in two small domains along the anterior fold marking the future labral buds (Figure 4.13A). While expression domains in the gnathal and thoracic segments were added one by one the, expression domain marking the two labral buds increased (Figure 4.13C). Later during development, the expression domain in the labrum was localized in the most distal portion of the labral bud (Figure 4.13E, G). After knockdown of Tc-mibl a weak domain of $T c-D l l$ expression remained in the labrum but the expression level was strongly decreased in 
comparison to the wild type throughout development (compare arrowheads in Figure 4.13A, $\mathrm{C}, \mathrm{E}$ and $\mathrm{G}$ with $\mathrm{B}, \mathrm{D}, \mathrm{F}, \mathrm{H})$. Alterations of expression domains of the gnathal and trunk appendages were not observed between wild type and RNAi embryos (compare open arrowheads in Figure 4.13A, C, E and G with B, D, F, and H).

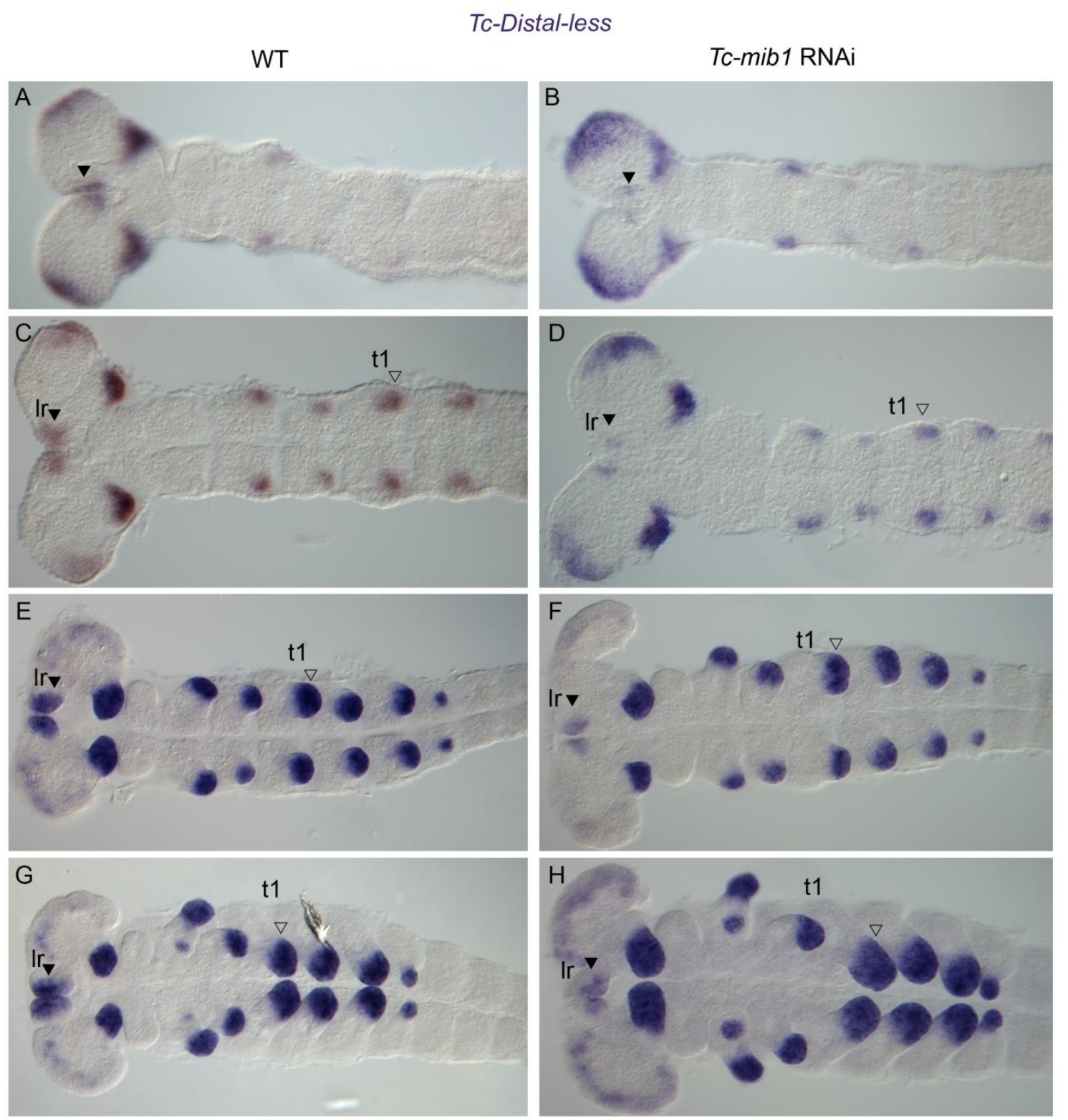

Figure 4.13: Expression of $T c$-Dll was affected after $T c$-mib1 RNAi in the labrum throughout development - Anterior to the left. Embryos in one row are of similar stages of development. (A, C, E, G) Expression of $T c-D l l$ in wild type embryos. (B, D, F, H) Expression of $T c-D l l$ in RNAi embryos. (A) In wild type $T c$-Dll expression was first detected inside the AMR along the anterior fold (arrowhead). (B) After $T c$ mibl RNAi expression in the AMR was decreased (compare arrowheads in A with B). (C, E) As development proceeded expression was added in the segments comprising antennae, gnathal and thoracic appendages ( $t 1)$. Expression in the labrum (lr) was now as much intense as in the other segments. (D, F) The expression domain in the labrum was decreased (compare arrowheads in $\mathrm{C}, \mathrm{E}$ with $\mathrm{D}, \mathrm{F}$ ) but not in the trunk appendages (compare open arrowheads in C, E with D, F). (G) In older elongating embryos the domain of Tc-Dll in the labrum ranged from the most distal tip with an intense expression to a weaker expression domain in the posterior part of the labrum. (H) After Tc-mibl RNAi expression of $T c$-Dll is decreased in the labrum (compare arrowheads G with 
H). In contrast, the expression of $T c-D l l$ in the trunnk appendages was not affected after Tc-mibl RNAi. (compare open arrowheads in $\mathrm{G}$ with $\mathrm{H}$ ).

In contrast to $T c-w g$ and $T c-d a c, T c-D l l$ expression was not deleted but strongly reduced in $T c$-mibl RNAi embryos. In order to test the possibility that Notch signaling and Tc-Dll interact with each other, $T c-D l l$ was knocked down and $T c-S e r$ staining was performed. The mutant phenotype of Tc-Dll was reproduced with knockdown (Beermann et al., 2001, 8). RNAi resulted in the reduction of distal structures of gnathal and trunk appendages and the antennae were completely missing (Figure 4.14B). In addition, $T c-D l l$ RNAi cuticles lacked almost the complete labrum tissue but the bristle of the clypeus remained (compare A with B). In RNAi embryos Tc-Ser expression was reduced in size (compare arrowheads Figure 18C, E, $\mathrm{G}$ with $\mathrm{D}, \mathrm{F}, \mathrm{H})$. However, the labrum tissue in $T c$-Dll RNAi embryos was also reduced in size (compare arrows in Figure 18E with F).

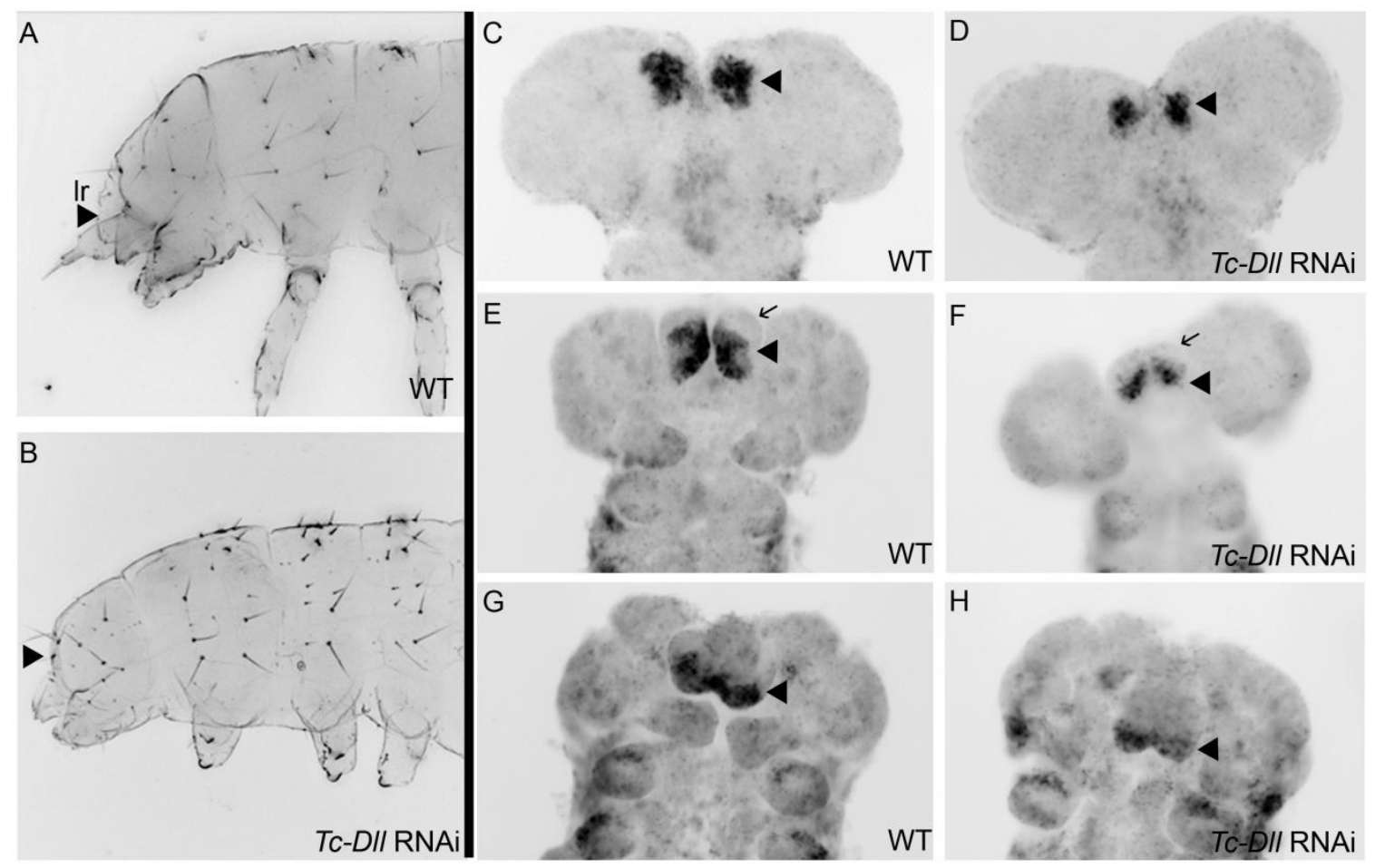

Figure 4.14: $\boldsymbol{T} \boldsymbol{c}$-Ser expression reduced in the labrum in $\boldsymbol{T} \boldsymbol{c}$-DIl RNAi - (A) Wild type cuticle of Tribolium first instar larvae (B) Cuticle after $T c-D l l$ RNAi (B). Anterior is to the left. (C, E, G) Wild type embryos. (D, F, H) RNAi embryos (D). (C-H) Embryos stained with Tc-Ser (TSA-Dylight550). Anterior to the top. Embryos in one row are of similar age. (B) After $T c$-Dll RNAi the antennae was completely missing and the distal portion of trunk and gnathal appendages except for the mandible was reduced. Additionally, the labrum tissue was highly reduced but the bristle marking the clypeus was still visible in most cuticles (compare arrowhead in A with B). (D, F, H) Expression of Tc-Ser in RNAi embryos was decreased in the distal portion of the labrum (compare arrowheads in C, E, G, with D, F, H). (F) In RNAi embryos a reduction of the labral buds was observed (compare arrows in $\mathrm{E}$ with F). 
In wild type embryos $T c-d p p$ is expressed in the labrum anlagen (arrows in Figure 4.15A, C, and E) and the distal part of the outgrowing legs (open arrowheads in Figure 4.15A, C, and E). After Tc-mibl RNAi the expression in the labrum was reduced in advanced elongating embryos (compare arrows in Figure 19). Shortly after outgrowth of the labrum, expression of $T c-d p p$ was only slightly affected in the most distal portion (compare arrowheads in Figure 4.15A, C, and E with B, D, and F). The proximal weak expression of Tc-dpp in the labrum was unaffected throughout development (compare arrows in Figure 4.15 A, C, E with B, D, and F). Like with $T c$ - $D l l$, no alteration was detected in the expression of $T c-d p p$ in the legs after Tc-mibl RNAi (open arrowheads in Figure 4.15). However, this slight alteration of $T c$ $d p p$ expression might be due to the loss of the labrum structure. Cuticle analysis of sibling embryos confirmed that strong phenotypes were analyzed.

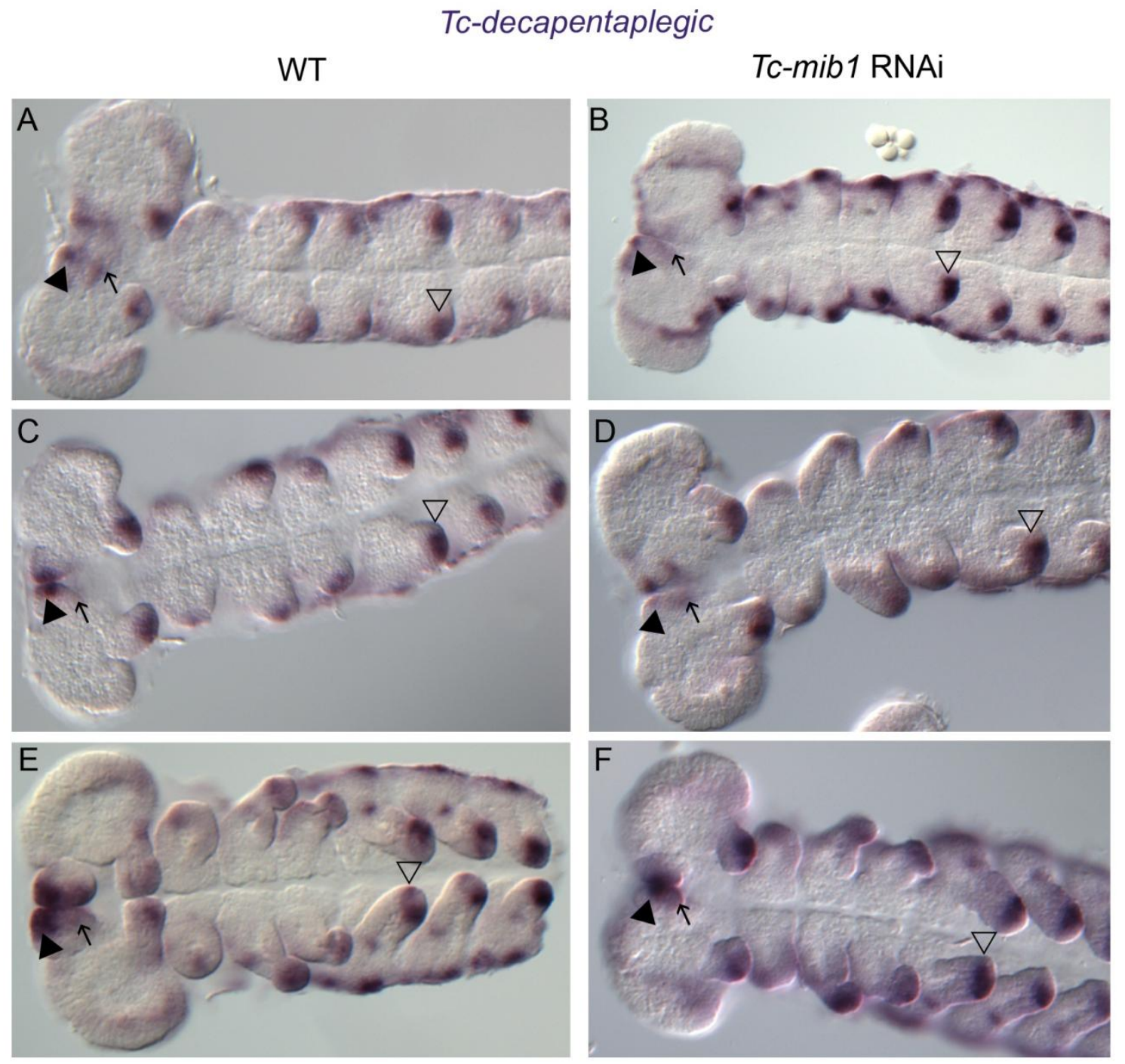

Figure 4.15: Expression of $\boldsymbol{T} \boldsymbol{c}$ - $\boldsymbol{d p p}$ was slightly affected in the labrum after $\boldsymbol{T} \boldsymbol{c}$-mib1 RNAi - (A, C, E) Wild type embryos stained with $T c-d p p$. (B, D, F) Tc-mibl RNAi embryos. (A) While labrum and appendages started to grow out, a localized expression of $T c-d p p$ was detected in the distal portion (arrowhead) and the most 
proximal part of the labrum (arrow). (C, E) During elongation expression increased with a more intense domain in the distal ventral portion (arrowhead) and a weaker proximal domain (arrow). (B, D) In RNAi embryos, shortly after limb growth initialization, the distal part of one labral bud showed a reduction in expression of $T c$ $d p p$ (compare black arrowheads in A, C with B, D). (F) At later stages, the domain was reduced in size but was still detectable after Tc-mibl RNAi (black arrowhead in F). (A-F) After Tc-mibl RNAi no difference in the expression domain of $T c-d p p$ In the outgrowing legs in comparison to the wild type was observed (compare open arrowheads).

\subsubsection{Notch signaling regulates cell proliferation during early labrum formation}

The role of Notch signaling to induce cell proliferation is well studied in Drosophila imaginal discs (Go et al., 1998). In addition, also in the spider Cupiennius salei cell proliferation via Notch signaling is necessary for the formation of the legs (Prpic and Damen, 2009). In order to investigate whether the reduced labrum anlagen in Tc-mibl RNAi embryos was due to reduced cell proliferation during outgrowth of the labrum, I etablished the EdU cell proliferation assay in Tribolium castaneum embryos via injection. With this method, detection of DNA synthesis in proliferating cells is visualized by a fluorescent azide in a $\mathrm{Cu}(\mathrm{I})$ catalyzed [3 + 2] cycloaddition reaction (Rostovtsev et al., 2002; Tornøe et al., 2002) after incorporation of EdU during the S-phase of the cell cycle (Salic and Mitchison, 2008). An increased number of proliferating cells in the labrum was visualized in 19-20 h old wild type embryos after three hours of EdU treatment (arrowheads in Figure 4.16A, C). After Tc-mib1 RNAi, proliferation was reduced in the labrum anlagen (compare arrowheads in Figure 4.16 A-D) but not in the outgrowing legs at this time point of development (open arrowheads in Figure 4.16A-D). For quantification, all proliferating cells marked by EdU incorporation were counted in 22 wild type and 38 Tc-mibl RNAi embryos. Three different areas in the embryo were selected for counting: The first area compromised both outgrowing labral buds (blue area in Figure 4.16A). In the cuticle phenotype of Tc-mibl RNAi treated animals no clear reduction of the head size was detected. Moreover, the embryonic phenotype showed no alteration in the head lobe. Therefore, the head lobe was selected as negative control. Only the anterior half of the head lobes was chosen since most RNAi embryos showed slight alterations of the orientation of the head lobes due to the reduction of labrum tissue (grey in Figure 4.16A). Therefore, no consistent separation between head lobe and antenna anlagen could be done in the posterior part. The third area of interest was the first thoracic leg pair (purple in Figure 4.16A). Counts of the left and right side of each embryo were summed up and the mean values were used for the determination of alteration in the number of proliferating cells between wild type and Tc-mibl RNAi embryos (see section 3.6.9 for 
statistical analysis). No significant difference could be detected in the counted area of the head lobe ( $\mathrm{p}$-value $=0.1102)$ after knockdown (Figure 4.16E). In addition, no significant alteration was detected in the outgrowing leg pair ( $\mathrm{p}$-value $=0.2342$ ) at this time point of development (Figure 4.16E). In contrast, the outgrowing labrum showed a significant reduction in proliferating cells after $T c-m i b 1$ RNAi $\left(\mathrm{p}\right.$-value $=3.695 \mathrm{e}^{-07}$ ) (Figure 4.16E). 

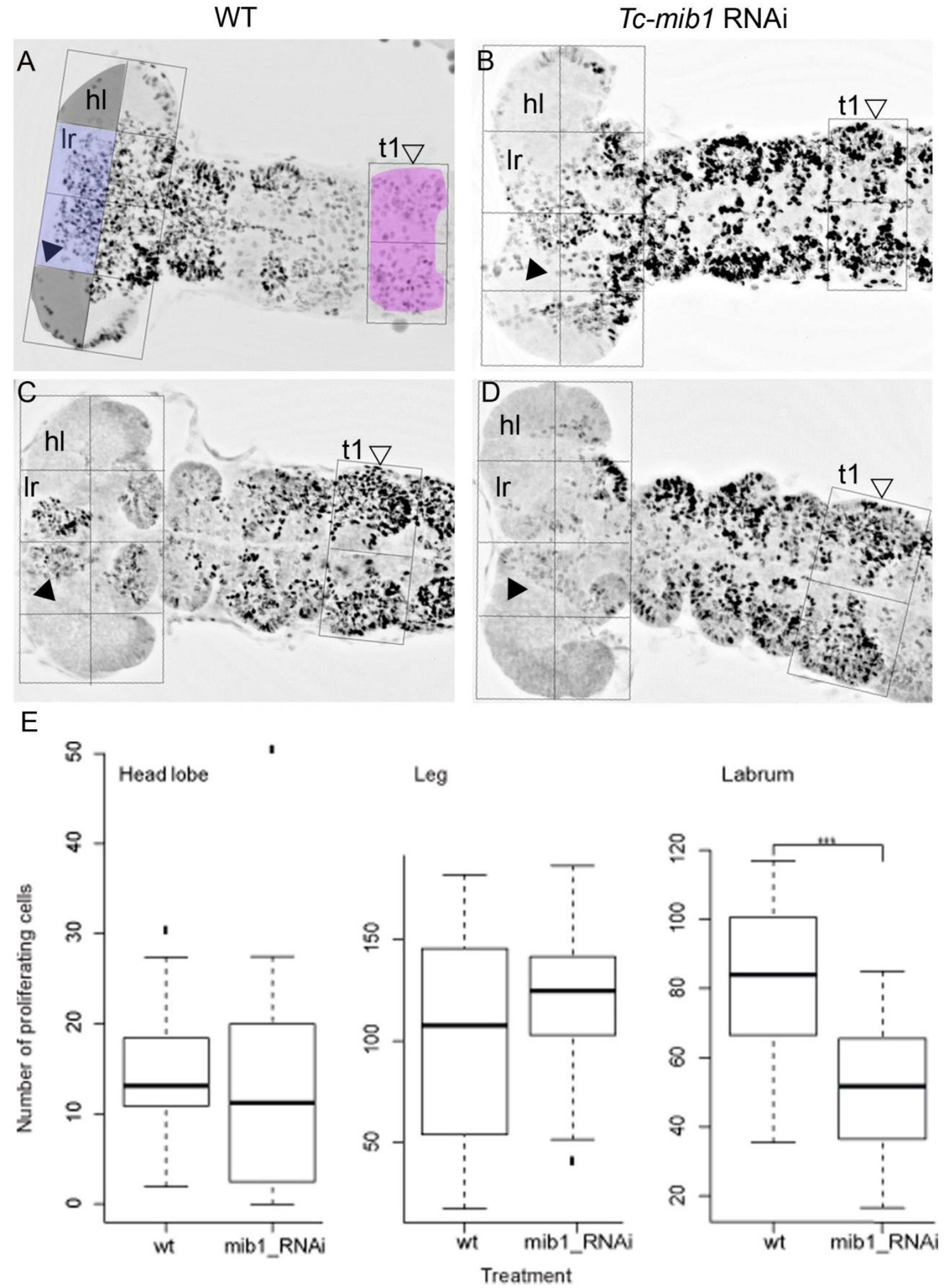

Figure 4.16: RNAi of $\boldsymbol{T} \boldsymbol{c}$-mib1 leads to reduction of cell proliferation in the labrum - (A-D) Anterior is left. Embryos in one row are approximately the same age. Boxes mark the areas of the head and the thoracic segments which were analyzed. (A, C) Wild type embryos after EdU incorporation. (B, D) EdU incorporation after Tc-mibl RNAi. (A) Colored regions represent the different counted regions used in wild type and Tc-mibl RNAi embryos. The grey colored area marked the upper half of the head lobes, blue the two labral buds and purple the first leg pair. (E) Statistical analyses of counted cells in wild type and RNAi embryos. (A, C) In wild type embryos, proliferation of cells was concentrated in the labrum (arrowheads) and the outgrowing thoracic 
appendages (open arrowheads). (B, D) After Tc-mibl RNAi, proliferation was reduced in the labrum (compare arrowheads in A, C with B, D) but unaffected in the legs (compare open arrowheads in A, C with B, D). (E) Statistical analysis revealed that the number of proliferating cells in the head lobes ( $\mathrm{p}$-value $=0.2342)$ and the first leg pair ( $\mathrm{p}$-value $=0.1102$ ) showed no significant alteration between wild type and RNAi treated embryos. In contrast, after knockdown of $T c-m i b 1$ the cell proliferation in the labrum was significantly reduced (p-value $\left.=3.695 \mathrm{e}^{-07}\right)$. $\mathrm{lr}=$ labrum; $\mathrm{t} 1=$ thoracic segment 1 ; $\mathrm{hl}=$ head lobe

\subsubsection{Tissue loss of labrum after knockdown not due to increased cell death}

The regulation of tissue-size underlies not only the process of cell division but also a controlled cell death during development. In order to test if enhanced cell death played a role, apoptotic cells were detected by cleaved Drosophila Dcp-1 antibody staining in wild type and Tc-mibl RNAi embryo. During early elongation both wild type embryos and dsRNA treated embryos showed a similar amount of dying cells in a diffuse pattern throughout the whole embryonic tissue (compare Figure 4.17A and B). Shortly after outgrowth of labral buds and appendages enhanced cell death was observed in the head and the midline in the developing nervous system of the embryo (arrows in Figure 4.17C, D). After Tc-mib1 RNAi no obvious alteration of the labrum and leg could be detected at this time point of development (compare Figure 4.17C with D).

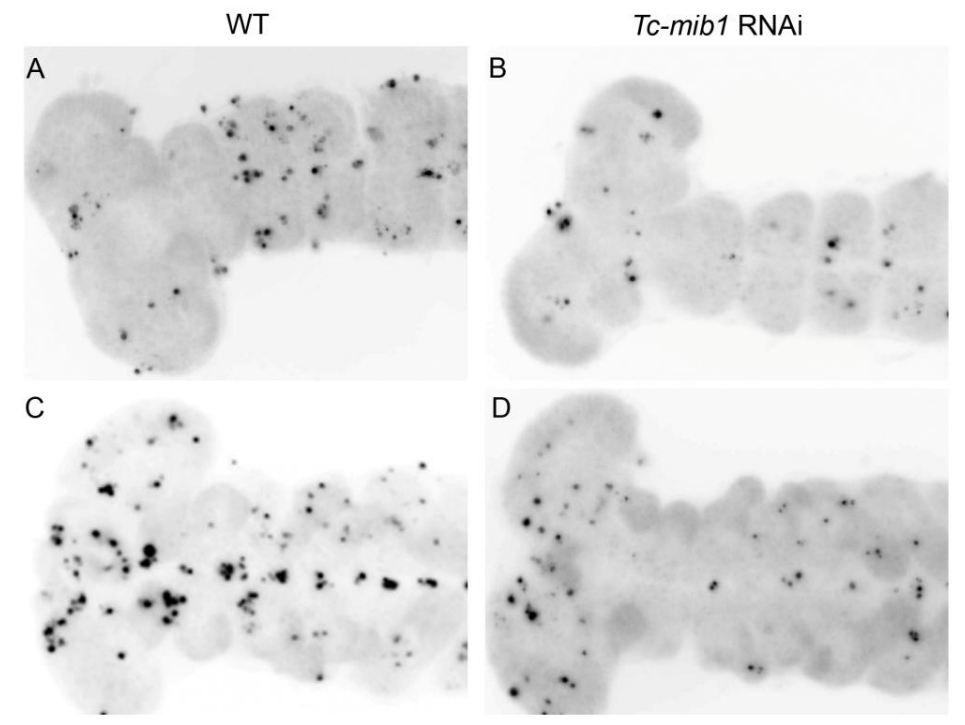

Figure 4.17: Cell death was
not enhanced after Tc-
mib1 RNAi - Anterior to the
left. A, C) Wild type
embryos showed a number
of dying cells throughout
development. (C) In older
embryos a higher number of
apoptosis especially in the
developing nervous system
was detected. (B, D) After
RNAi no enhancement of
cell death could be detected
in the labrum and the legs at
both time point of
development. 


\subsection{Involvement of the GATA transcription factor $\boldsymbol{T c}$-grain in labrum formation}

\subsubsection{Identification of iB_03552 as Tc-grain (Tc-grn)}

AUGUSTUS gene predictions revealed that the dsRNA fragment with the ibeetle ID number iB_03352 targets a gene with the number TC002315. BLAST analysis of the protein sequence against non-redundant protein sequences of Drosophila melanogaster identified the gene grain as the closest fly homolog which was also confirmed by phylogenetic analysis using the neighbour joining method (Figure 4.18). The annotation ID of this gene was CG9656-PB with the Flybase ID FBpp0289317. In Drosophila this gene is also known as GATAc, dGatac and gra and encodes a GATA transcription factor. In the following sections TC002315 will be referred to as Tc-grain (Tc-grn), respectively.

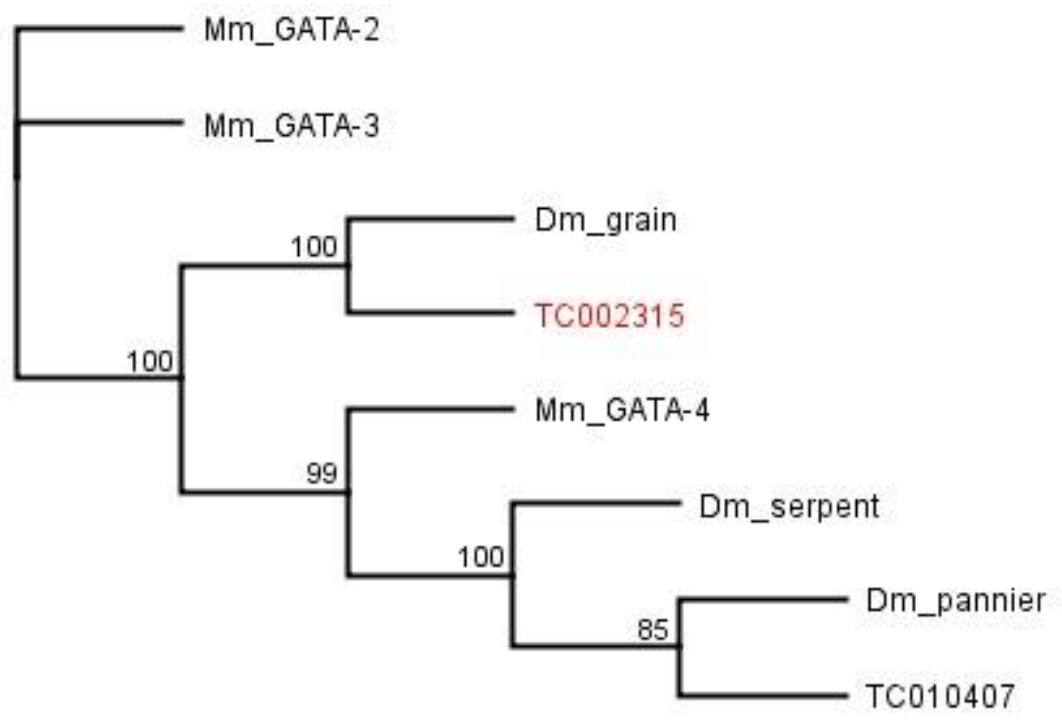

Figure 4.18: Tc-grain is targeted by iB_03552 (TC002315) - Tree and image created with Geneious version 7.0. Phylogenetic analysis of the protein sequence showed that the gene TC002315 is the single ortholog to Dm-grain. The paralog found in Tribolium (TC010407) clustered with Dm-pannier. Dm= Drosophila melanogaster; $\mathrm{Mm}=$ Mus musculus.

During rescreen analysis, two non-overlapping fragments did not reproduce the annotated phenotype but resulted in lethality and sterility. However, re-injection of the original iBeetle fragment into the strain pig-19 reproduced the results. Therefore, the two NOFs were analyzed using E-RNAi on the website of the German Cancer Research Center (DKFZ) (http://www.dkfz.de/signaling/e-rnai3/) (Horn and Boutros, 2010). Both fragments targeted 
other genes (fragment 1: TC011229, TC005779; fragment 2: TC011229, TC005779, TC002343, TC002364 and TC011219). The gene FK506-bp1 was found to be the closest fly homolog to TC005779. In Drosophila, phenotypic data described high lethality for this gene (flybase). In order to exclude possible off targets a third fragment was synthesized by ERNAi which did not target any other gene and was non-overlapping with the iBeetle fragment. This non-overlapping fragment reproduced all phenotypes found in the screen in both pig-19 and $S B$ strains.

\subsubsection{Functional analysis of Tc-grain}

Quantitative analysis of the cuticle phenotype showed that Tc-grn RNAi resulted in a decreased size of the head capsule with $100 \%$ penetrance (Figure $4.19 \mathrm{~B}^{1}$ ). In addition, the labrum was completely missing including all bristles of the labrum quartet with 100\% penetrance. In all Tc-grn RNAi cuticles the tracheal openings were reduced or missing (compare marked area in Figure 4.19A with B). The abdominal bristle pattern was also disturbed at lower frequency (74 \%, not shown). In the Tribolium first instar larvae distinct regions of the head are marked with a constant pattern of different setae and bristles (Schinko et al., 2008). Quantitative analysis in Tc-grn RNAi animals revealed that the pattern of setae and bristles was disturbed. In wild type larvae a row of four sensillae (bell row) is found at dorsal lateral position near the junction separating the head from the trunk. After Tc-grn RNAi the number of sensillae was only reduced with $15 \%$ penetrance (Figure $4.20 \mathrm{~B}$ ) but the arrangement of these sensillae was disturbed in $88 \%$ (compare yellow and orange dots in Figure 4.19A with B). Dorsal to the bell row the vertex triplet is located compromising posterior, anterior and ventral setae shaped as a triangle (purple triangle in Figure $4.19 \mathrm{~A}^{2}$ ). After Tc-grn knockdown mostly the anterior seta was missing (81\%, Figure 4.20B). Since RNAi animals lacked labrum tissue no setae of the labrum quartet were found (Figure 4.20B). 

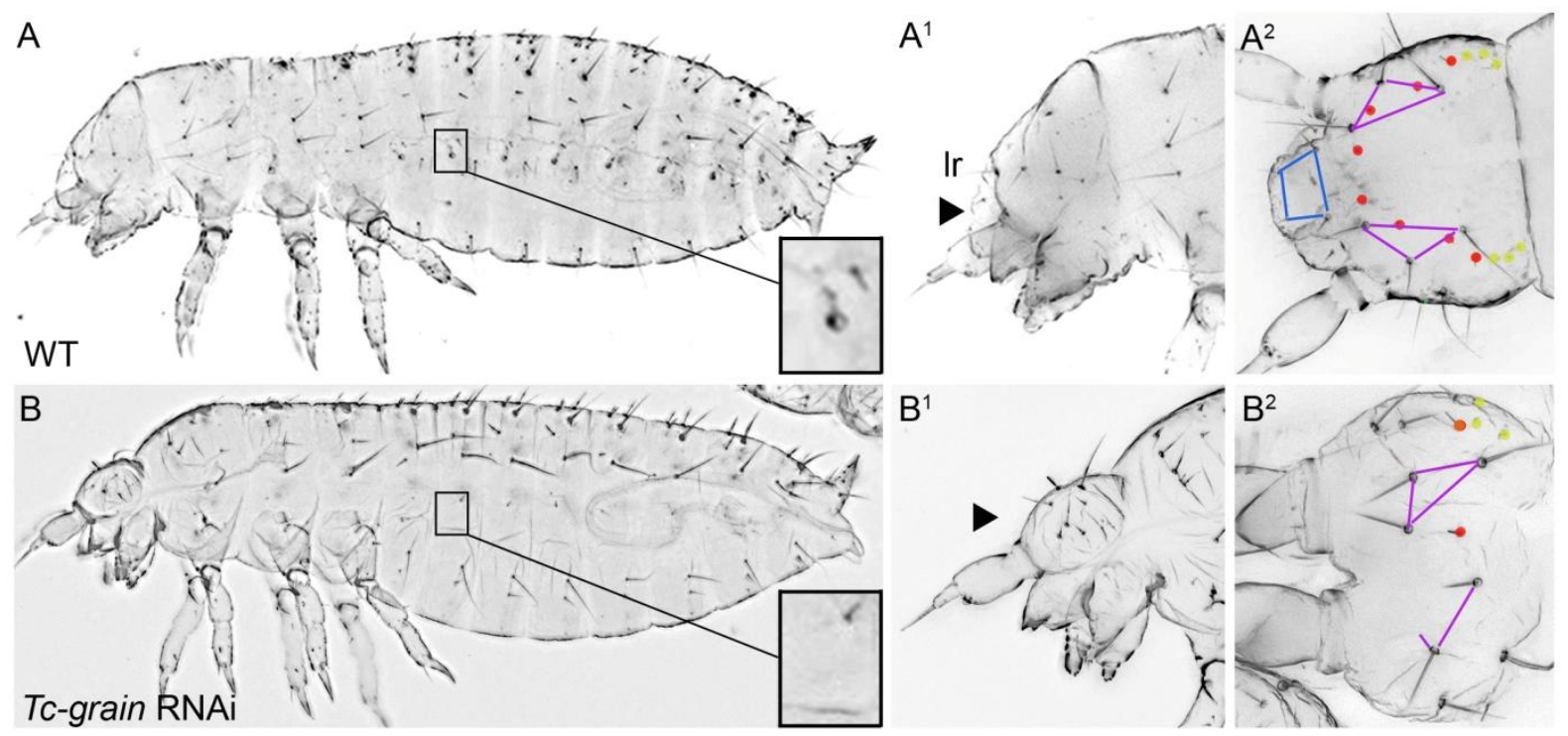

Figure 4.19: Cuticle phenotype after Tc-grn RNAi - Anterior is to the left in $\left(A-A^{1}\right.$ and $\left.B-B^{1}\right) .\left(A^{2}, B^{2}\right)$ Dorsal view of cuticle heads. (A) Wildtype cuticles. (B) Cuticles of Tc-grn RNAi animals. The groups of setae are indicated by colored lines (purple triangle $=$ vertex triplet; red and three yellow dots= bell row; blue quadar= labrum quartet; red dots= vertex triplet bristle). (B) In Tc-grn RNAi cuticles most of the tracheal openings were lost (compare detailed view of marked area in $\mathrm{A}$ with $\mathrm{B}$ ). $\left(\mathrm{B}^{1}\right)$ In addition, the size of the head capsule was highly reduced and the labrum (lr) tissue was completely missing (arrowhead in $\left.\mathrm{B}^{1}\right)$. $\left(\mathrm{B}^{2}\right)$ In the head, the bristle pattern of the labrum quartet and bristles in the region of the vertex triplet were lost (compare red dots in $\mathrm{A}^{2}$ with $\mathrm{B}^{2}$ ). The vertex triplet was shifted and the anterior setae were often missing (compare purple triangle in $\mathrm{A}^{2}$ with lines in $\mathrm{B}^{2}$ ). The number of bell row bristles was not reduced but shifted (compare $\mathrm{A}^{2}$ with $\mathrm{B}^{2}$ ).

Moreover, quantitative analysis of the head bristle pattern revealed that the triplet bristle of the vertex triplet was highly reduced in numbers and the antenna basis bristle could not be found in $91 \%$ of the analyzed cuticles (compare red dots in Figure $4.19 \mathrm{~A}^{2}$ with $\mathrm{B}^{2}$ ). In wild type, the lateral portion of the head is marked by three setae called the gena triplet. In RNAi cuticles, mostly the posterior seta was missing (Figure 4.20B). Furthermore, in wild type the so called maxilla escort is located below to the gena triplet marking the bulge of the maxilla segment with three setae. After RNAi all three setae were mostly lost (Figure 4.20B). The dorsal ridge marking the junction between head and trunk was not affected after Tc-grn RNAi.

Taken together, not only the labrum including the dorsal portion of the cuticle head but also tissue of the lateral portion was missing in Tc-grn RNAi cuticles (Figure 4.20A ${ }^{3}$ ). 


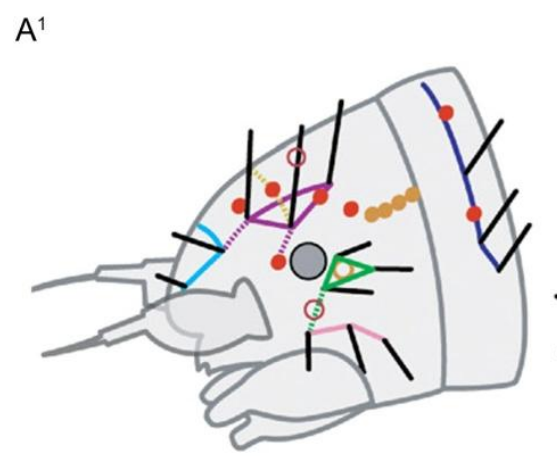

$A^{2} \quad A^{3}$

B
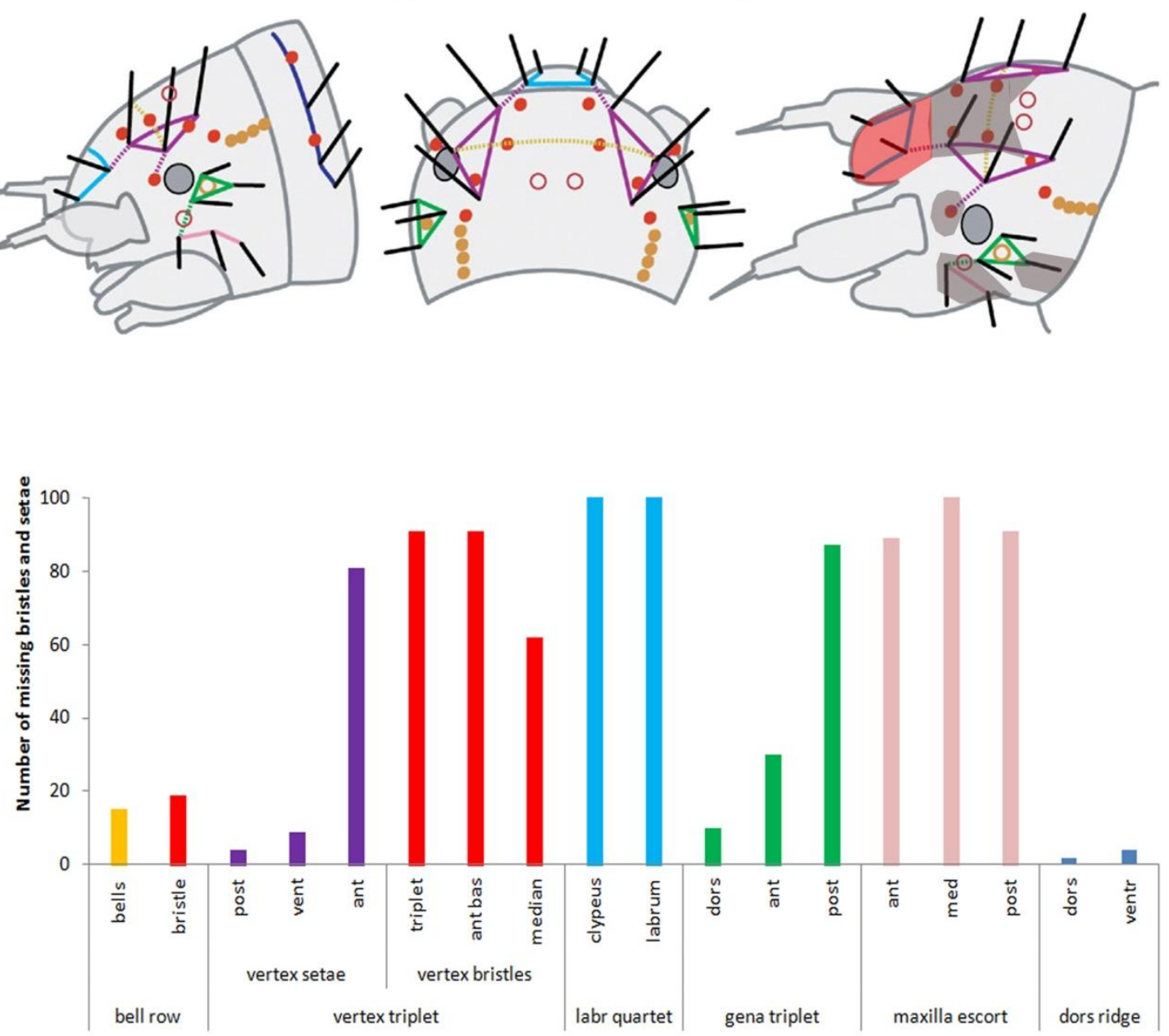

Figure 4.20: Quantitative analysis of the head bristle pattern after $T c$-grn RNAi - $\left(\mathrm{A}^{1}-\mathrm{A}^{3}\right)$ Schematic overview of the Tribolium head with colored bristle pattern (picture taken from (Schinko et al., 2008)). $\left(\mathrm{A}^{3}\right)$ the red area marks the deletion of labrum tissue. Grey areas marking presumptive deletions of head cuticle after $T c$ grn RNAi. (B) Quantitative analysis of the head bristle pattern after Tc-grn RNAi. The percentage of cuticles that lacked the respective bristle or setae is given. Bars were colored according to the color code seen in (A). After Tc-grn RNAi, the bell row and the dorsal ridge were mostly unaffected in the number of bristle and setae. In contrast, the vertex triplet was disturbed. In around $80 \%$ of all cuticles the most anterior seta was missing. Additionally, the bristle triplet and the antenna basis bristle were lost in 90\% of analyzed bristle pattern. Furthermore, the posterior seta of the gena triplet was missing in over $80 \%$ percent. All cuticles of Tc-grn RNAi lacked the labrum quartet and in over $90 \%$ the maxilla escort was missing.

\subsubsection{Embryonic phenotype of $T c$-grn}

In order to see if Tc-grn RNAi showed a similar phenotype in the cuticle and embryos, nuclear staining with Hoechst was investigated in RNAi embryos. Surprisingly, analysis of Tc-mibl RNAi embryos already showed that the labrum appeared to form properly initially but stopped to increase in size over time. While in RNAi cuticles the labrum was completely 
lost, in embryos the labrum tissue was still visible. Interestingly, in contrast to the wild type situation these two labral buds were fused precociously (compare arrowheads in Figure 4.21 A with B).

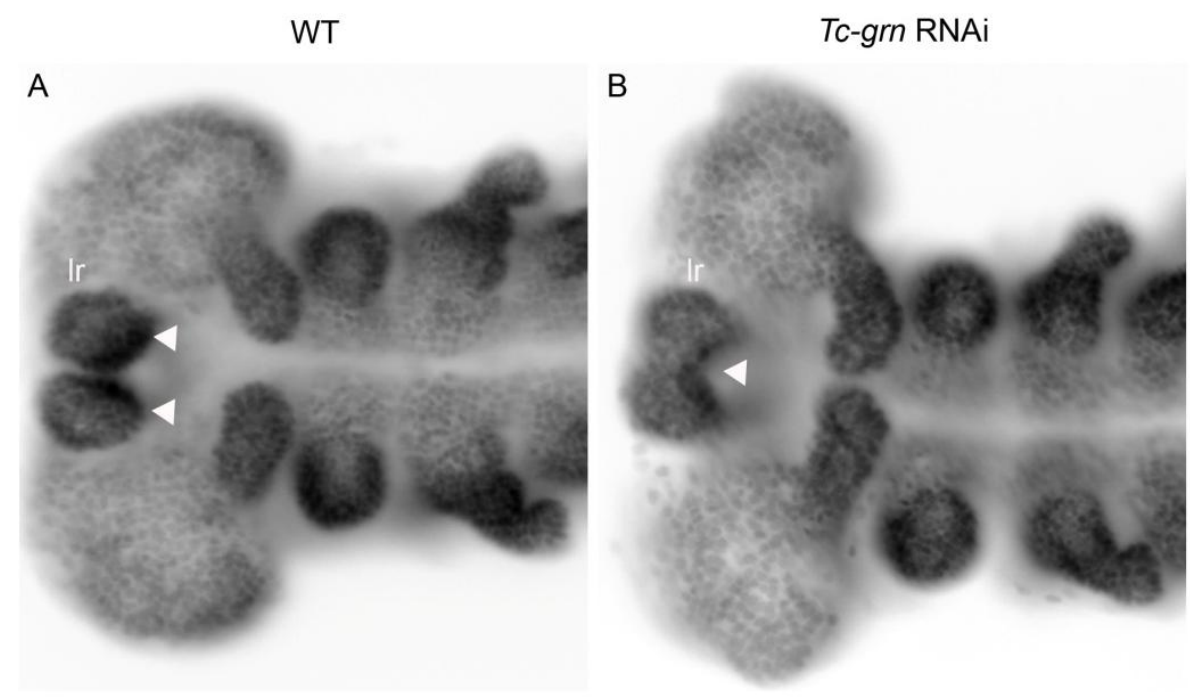

Figure 4.21: The labral buds are fused precociously in Tc-grn RNAi embryos - Anterior is to the left. Nuclei stained with Hoechst. (A) Wild type embryo. (B) Tc-grn RNAi embryo. (B) During embryonic development the labrum was present after Tc-grn RNAi. (A,B) In contrast to the wild type labral buds, which were still separated at this time of development (arrowheads in A), RNAi embryos showed already a fusion at the distal tip of the labrum anlagen (compare arrowheads in A with B).

\subsubsection{Tc-grn is expressed in the head throughout development}

Analysis of grain in Drosophila showed that expression occurred during early gastrulation in the procephalic head region and later during embryonic development in the developing posterior spiracles, central nervous system, midgut primordia, the optic lobes and the tip of clypeolabrum (Lin et al., 1995). Since expression of Tc-grain was not described in Tribolium so far, in situ staining was performed. Expression of Tc-grn was already detected in early germ rudiments marking serosa cells in the anterior portion (open arrowhead in Figure 4.22A). In late germ rudiments three distinct expression domains were detected; the first still visible in serosa cells (open arrowhead in Figure 4.22B), the second marking the head lobes (arrow in Figure 4.22B) anterior-lateral and the third localized at the middle plate as a patch of cells (Figure 4.22B). This ventral patch of stained cells expanded during early elongation of the embryos (Figure 4.22C, E) and vanished at later stages of development (Figure 4.22F). While expression of a few serosa cells was still visible in early elongating embryos, 
expression of Tc-grn was not only observed in the head lobes (arrow in Figure 4.22D), but also in the amnion (asterisk in Figure 4.22D). During early elongation the expression domain of Tc-grn became more restricted to the most anterior portion of the head lobes (Figure 4.22E) and was now also visible along the anterior fold (arrowheads in Figure 4.22E). Later during development, the expression in the head lobes split into three separated domains (Figure 4.22F). One marking the ocular region of the embryo (circle in Figure 4.22F), the second became located lateral to the labrum presumably marking neurogenic tissue (cross in Figure 4.22F) and inside of the outgrowing labrum (arrowhead in Figure 4.22F). In addition, an additional domain arose posterior to the ocular domain as a line of cells lateral to the antennae (Figure 4.22F). Both expression domains of the ocular region and the one surrounding the antennae were stable during later development (Figure 4.22G). While the labral buds started to grow and were clearly visible as two bulbs, the expression of Tc-grn split up. A cap of expression was visible in the most distal portion of the labrum anlagen located lateral of the labrum bulb (arrowhead in Figure 4.22G ${ }^{1}$ ). Beyond that, a domain in the proximal part of the labrum arose (arrowhead in Figure 4.22G ${ }^{1}$ ) and Tc-grn was also detected at the ventral tissue connecting the head lobes (Figure $4.22 \mathrm{G}^{2}$ ). 

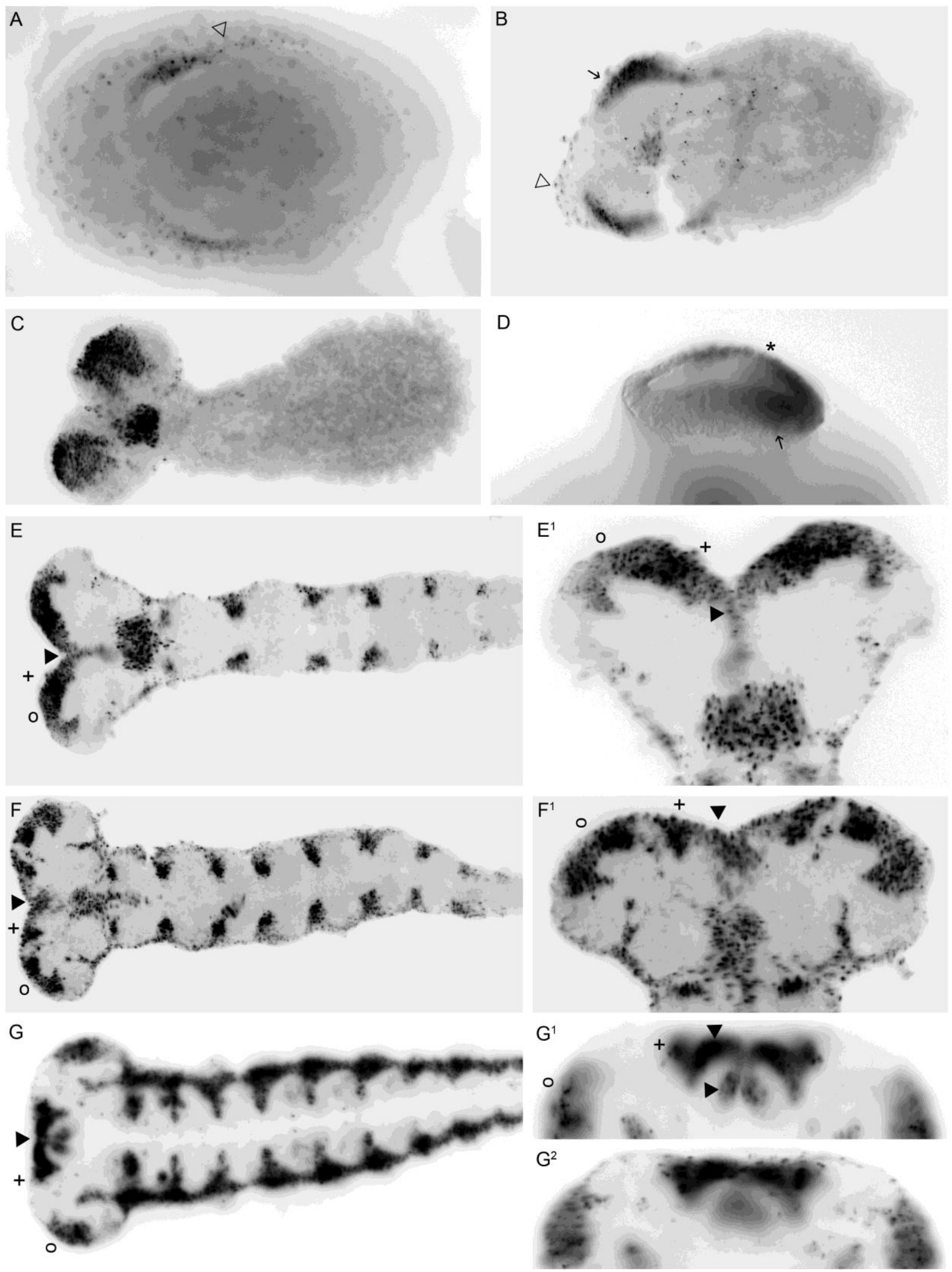

Figure 4.22: Expression of $\boldsymbol{T} \boldsymbol{c}$-grain during development - (A-C and E-F) anterior is to the left. (D) Detailed view of one head lobe (approximately the same age as in $C$ ). $\left(E^{1}-G^{2}\right)$ Detailed view of the head of the embryos seen on the left with anterior to the top. $\left(\mathrm{E}^{1}\right.$ and $\left.\mathrm{G}^{2}\right)$ Ventral view of the heads. (A) Expression of Tc-grn started already in early germ rudiments marking serosa cells (open arrowhead). (B) Later, expression was still visible in serosa cells (open arrowhead), but also in the head lobes (arrow) and in a small domain ventral in the middle plate marking mesodermal cells. (C, D) In early germ bands the expressions of the two lateral domains were still located in the anterior tip of the head lobes and the domain of the middle plate got more expanded. In addition, 
expressed also occurred in the amnion (asterisk). (E) Later in development, the expression domains in the head lobes were most anterior (neurogentic tissue marked with a cross, ocular region is marked with a circle) and fused along the anterior fold which marks the labrum anlagen and stomodaeum (arrowhead). Furthermore, the expression of Tc-grn now started also in the trunk segments. (F) As elongation proceeded, the ventral expression in the middle of the head started to vanish and the domains in the anterior head lobe split. Now a few cells marking the anterior tip of the outgrowing labrum anlangen (arrowhead) showed Tc-grain expression. In the ocular region (circle) of the head lobes, a defined area of Tc-grn expressing cells became visible. Posterior to the ocular domain a small line of expressing cells was detected surrounding the antennae. (G) In older elongating embryos the expression of the trunk, which started as patches (see E and F), became connected along two lateral domains. In the head a precise domain of the Tc-grn expression was observed in the ocular region (circle). $\left(G^{1}\right)$ The expression in the labrum (arrowhead) was visible as a cap concentrated at the most distal lateral portion. In addition, a less intense domain was also found more proximal of the labral buds. $\left(\mathrm{G}^{2}\right)$ Expression of $T c$ - $g r n$ was also observed at the connection between both head lobes.

\subsubsection{Tc-grn is not an upstream regulator of the AMR network}

In order to test if Tc-grn acted upstream of the gene regulatory network of the AMR, staining of genes which were expressed inside this region and the surrounding tissue was performed in Tc-grn RNAi embryos. The expression domains of Tc-cnc, Tc-croc and Tc-six4 were not affected after Tc-grn RNAi (compare Figure 4.23A-C with E-H). Both expression domains inside the labrum and the surrounding tissue, marking the neurogenic tissue, were detected after Tc-grn RNAi. Nevertheless, a slight alteration was observed because the expression domain in the neurogenic tissue was shifted towards anterior (arrows in Figure 4.23D, I). The proximal expression domain inside the labrum was shifted towards anterior, too (dotted lines in Figure 4.23D, I).

However, this alteration was more likely due to morphological alterations of the labrum and head lobe tissue. In summary, no evidence for Tc-grn acting upstream of these genes was found. 


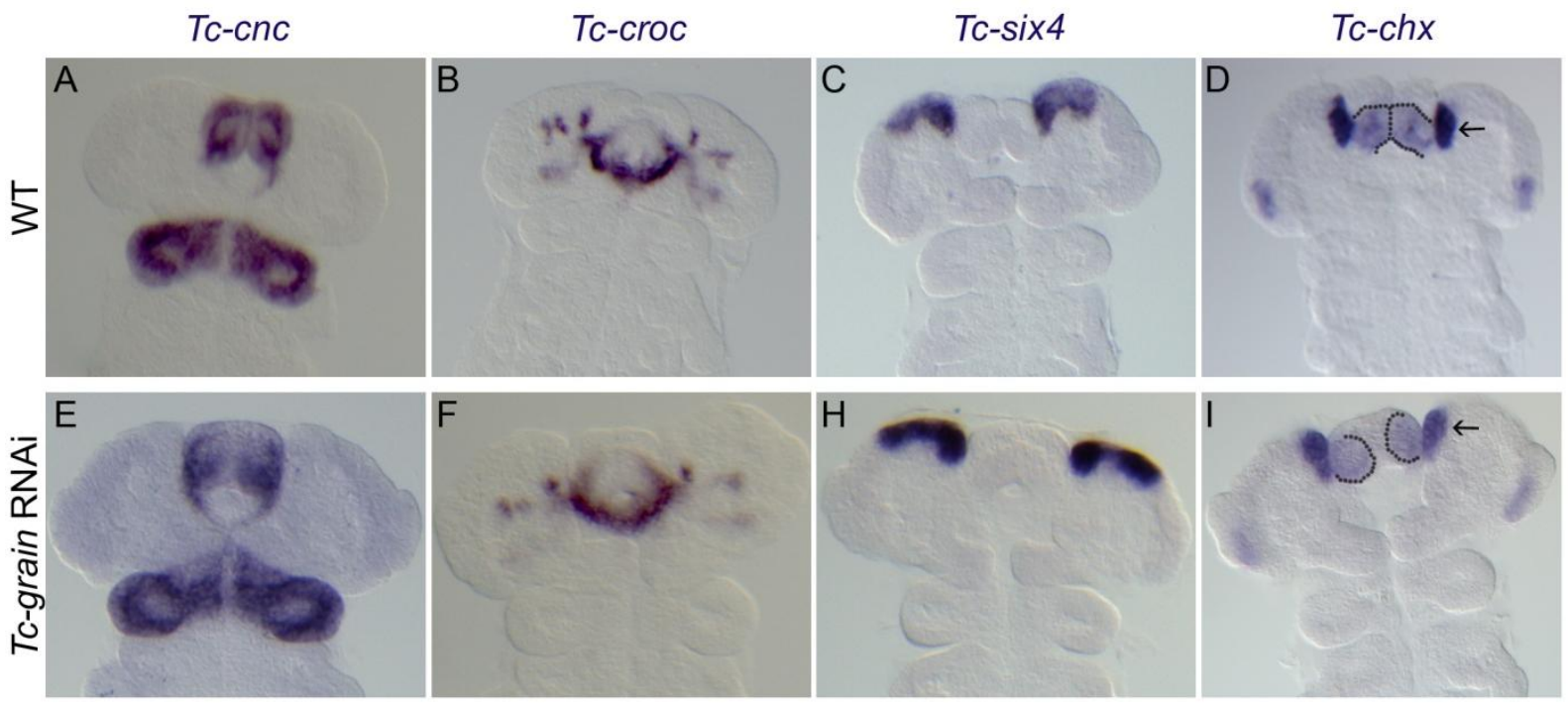

Figure 4.23: Expression of AMR marker genes not affected after $\boldsymbol{T} \boldsymbol{c}$-grn RNAi - Anterior to the top. (A -D) Wild type embryos stained for Tc-cnc, Tc-croc, Tc-six4, Tc-chx. (E-I) Corresponding expression in Tc-mib1 RNAi embryos of similar age. (A-C and E-H) Pictures provided by Tobias Vollmer. (E-H) Expression of Tc-cnc, $T c-c r o c$ and $T c$-six4 was not affected after Tc-grn RNAi (compare A-C with E-H). (I) The expression domains $T c$-chx in the labrum and neurogenic tissue of was not affected after $T c$-grn RNAi. However, the expression in the neurogenic tissue and the labrum was slightly shifted towards anterior after $T c$-grn RNAi (compare arrows and dotted lines in $\mathrm{D}$ with I).

\subsubsection{Tc-grn is involved in the appendage regulatory gene network}

In situ stainings of Tc-grn RNAi embryos with AMR marker genes revealed that this gene was not acting upstream of this regulatory network. The appendage regulatory network is known to be involved in gnathal and trunk appendage and in labrum formation (Posnien et al., 2009a). In order to test if Tc-grn was involved in this regulatory network, in situ staining of $T c-w g, T c-d a c$ and $T c-D l l$ were performed in RNAi embryos. After knockdown of $T c-g r n$ the expression domain of $T c-w g$ and $T c-d a c$ was completely lost in the labrum (compare arrowheads in Figure 4.24A, C with B, D). Beyond that the expression level of Tc-Dll was decreased in the labrum after Tc-grn RNAi (compare arrowheads in Figure 4.24E with F). This results indicated that Tc-grn might act upstream of the appendage regulatory network in the labrum. 

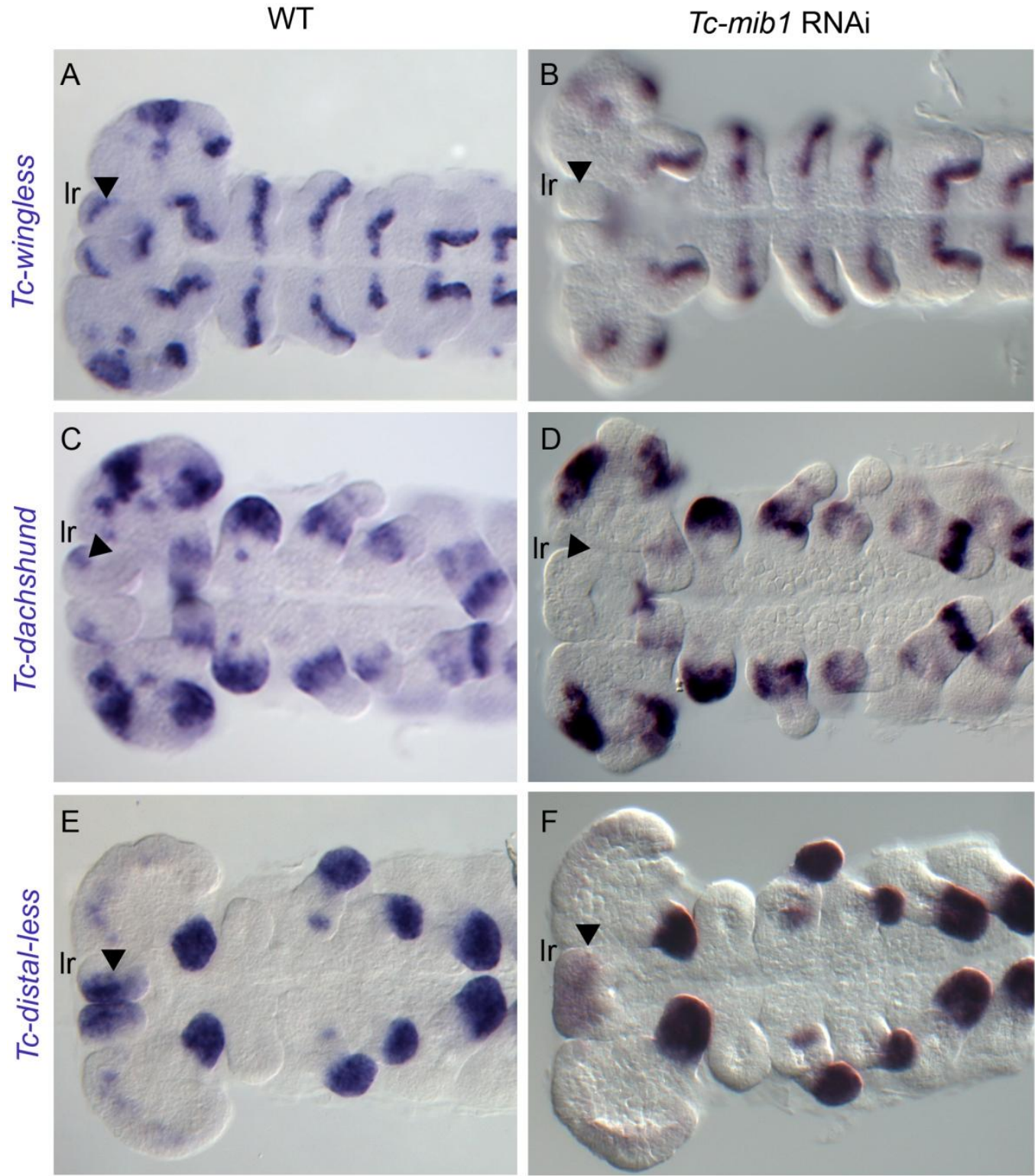

Figure 4.24: Expression of proximal- distal patterning genes in the labrum affected after Tc-grn RNAi - Anterior is to the left. Embryos in one column are of the same age. (A, C, E) Wild type embryos; (B, D, F) Tc-grn RNAi embryos. (B, D)Tc-wg and $T c$-dac expression in the labrum (lr) was completely lost after $T c$-grn RNAi (compare arrowheads in A, C with B, D). (F) After Tc-grn RNAi, expression of $T c$-Dll in the labrum was decreased (compare arrowheads in $\mathrm{E}$ with $\mathrm{F}$ ). 


\section{Discussion}

\subsection{Low number of new head development genes detected in the iBeetle screen}

The data collected during this work supports the notion that the iBeetle screen is an efficient approach to identify genes with novel functions (Schmitt-Engel et al. accepted). Using an unbiased screen, I was able to indentify five genes with novel functions in Tribolium in comparison to Drosophila knowledge. Two genes were not required for head patterning, but showed new functions in Tribolium, which could be interesting for other projects: The ortholog of Drosophila Dscam is known to be a cell adhesion gene with extensive alternative splicing. In Drosophila, Dscam is not only involved in many aspects of neurogenesis, but also in immunity (Schmucker and Chen, 2009; Schmucker et al., 2000; Watson et al., 2005). During my work, I identified an additional role in sensory organ formation in Tribolium. The ortholog of the Drosophila chromatin ATPase kismet (a trithorax group protein) is involved in activating Hox genes throughout development. Loss of function mutants phenocopy aspects of the Scr, $U B X$ and $A b d B$ mutants (Daubresse et al., 1999; Srinivasan et al., 2005; Srinivasan et al., 2008). In my work, I identified an additional function in dorsal closure of the trunk.

Interestingly, three candidates showed evidence to be involved in the formation of the labrum. Two genes were identified as the orthologs of Drosophila mibl and Serrate. Both genes are known to be components of the Notch signaling pathway. While Notch signaling contributes to a large number of different developmental processes (Andersson et al., 2011; ArtavanisTsakonas et al., 1999) a role in labrum formation was not reported so far. Furthermore, the ortholog of Drosophila grain turned out to have a major role in labrum formation, while in Drosophila it is mostly connected to neurogenesis (Garces and Thor, 2006; Zarin et al., 2012; Zarin et al., 2014) and for shaping the adult legs and the larval posterior spiracles via cell rearrangement. Although head phenotypes were also found in Drosophila, they were not studied (Brown and Castelli-Gair Hombría, 2000).

However, the number of identified genes contributing to head development was unexpectedly low (Schwirz, 2014). One explanation could be that most of the genes required for head development are already known. Additionally, the first screening phase of the iBeetle screen biased genes with a high expression. Likewise, some genes could be missed in this first phase and will be found in the next round. However, the unbiased screen in Tribolium at least 
complemented the candidate gene approach which already revealed that the regulatory network in the head differs between Tribolium and Drosophila. (Bucher et al., 2005; Fu et al., 2012; Kittelmann et al., 2013; Posnien et al., 2011b; Schinko et al., 2008; Schoppmeier and Schröder, 2005; Schröder, 2003; Schröder et al., 2000)

\subsection{Notch signaling is involved in labrum and leg formation}

During this work, knockdown of the two components of the Notch signaling pathway (TCmibl and Tc-Ser) resulted in severe defects of the labrum and the trunk appendages. Therefore, these data suggested that the Notch signaling pathway in Tribolium has a major role both in labrum and leg formation.

\subsubsection{Conserved function of Notch signaling in arthropod leg development}

As expected, Notch signaling plays a similar role in Tribolium leg patterning as in other arthropods. In Drosophila not only the location and number of joints in the adult legs, but also the growth of the segments in the leg is controlled by Notch signaling by a combinatorial activation by Ser and $\mathrm{Dl}$. The Notch pathway is acting downstream of a gene cascade that patterns the leg (see Figure 2.2) (Bishop et al., 1999; Celis et al., 1998; Lecuit and Cohen, 1997; Rauskolb, 2001; Rauskolb and Irvine, 1999). Within arthropods, the function of Notch signaling in the legs is highly conserved. Expression and functional analysis of the Notch signaling components in the spider Cupiennius salei (Chelicerate, which is the most basally branching arthropod) and Gryllus bimaculatus (cricket), an intermediate-germ band embryo, showed that Notch signaling mediates leg formation in a similar way to the fly Drosophila (Mito et al., 2011; Prpic and Damen, 2009). Previously, a putative role for Notch mediated leg formation via the ligand Delta was shown for Tribolium (Aranda, 2006). Additionally, it had been known that the ligand Tc-Ser is expressed in ring shaped domains marking the leg joints during late embryogenesis (Aranda et al., 2008; Beermann et al., 2004). This expression pattern is comparable to the expression pattern of Ser in the legs of both spiders and flies (Bishop et al., 1999; Prpic and Damen, 2009).

The data presented in my work indicated that the Notch pathway plays a similar role in Tribolium leg formation. The disruption of Notch signaling via the ligand Tc-Ser led to shortened legs, where the joints were missing and tibiotarsus and femur were completely fused. However, down regulation of the ubiquitin ligase Tc-mibl resulted in stronger phenotypes in the legs than Tc-Ser RNAi individuals (see Table 4.2). One explanation for a 
weaker malformation of the legs of Tc-Ser RNAi cuticles is that like in Drosophila, a combination of $T c-S e r$ and $T c-D l$ signals may be necessary to induce Notch signaling for leg development. The need of both ligands to induce Notch signaling in the leg seems to be conserved in Tribolium, because leg phenotypes are also reported in Tc-Dl RNAi background (Aranda, 2006). Moreover, during metamorphosis, the knockdown of Notch or either one of its ligands results in a failure of appendage joint development indicating that Notch signaling in the legs is activated by both ligands (Angelini et al., 2012a).

In Drosophila, Notch signaling is controlled by the leg gap genes Dll and dac (Rauskolb, 2001). The expression of both genes is dependent on a combination of $w g$ and $d p p$ signaling (Lecuit and Cohen, 1997; Rauskolb and Irvine, 1999). Likewise, analysis in the spider showed that Notch signaling is presumably acting downstream of these genes and that the leg gap genes are not dependent on Notch signaling (Prpic and Damen, 2009). In this work, I could demonstrate that the same is true in Tribolium.

\subsubsection{A novel role of Notch signaling in labrum formation}

The results of my work demonstrated that activation of Notch signaling via the ligand $T c-S e r$ is required for proper formation of the labrum during embryogenesis. In contrast, $T c-D l$ seems not to be involved in this process during embryogenesis, since knockdown of $T c-D l$ induces malformation of the gnathal appendages, but specific labrum defects are not reported (Aranda, 2006). Recently, studies of metamorphic labral axis patterning in Tribolium showed that depletion of $T c-N$ induces loss of the distal sclerite at low frequency. Depletion of $T c-N$ or $T c$ $D l$ also causes defects in the patterning of the epipharyngeal sensillum located at the ventral side of the labrum, while Tc-Ser is mostly connected to ventral appendage development (Angelini et al., 2012a; Smith et al., 2014). The role of Notch in embryonic labrum formation has not been studied in Drosophila. While head defects of Ser knockout animals were mentioned before, functional analysis concentrated mostly on the gnathal appendages where Ser is required for normal maxilla mouth hook morphogenesis (Wiellette and McGinnis, 1999).

The labrum phenotype of Tc-mibl and Tc-Ser was similar to the one of Tc-six3 and Tc-cnc, which are genes of the AMR patterning network (Kittelmann et al., 2013; Posnien et al., 2009a; Posnien et al., 2011b). Additionally, Tc-Ser expression started already in early elongating embryos in the AMR. In Drosophila, Ser expression starts at late stage 10 embryos localized in the clypeolabrum (Fleming et al., 1990; Thomas et al., 1991) slightly later at a 
similar stage than Tribolium, indicating that it could be involved similarly later in embryonic labrum formation. However, Notch does not appear to act in pattern formation of the AMR. FM® 1-43 staining of Tc-mibl RNAi embryos demonstrated that the initial outgrowth of the labral anlagen was not affected. These results suggest that Notch signaling in labrum formation plays a later role. Second, analysis of early pattering genes including $T c$-cnc and Tc-croc in Tc-mibl RNAi background showed no evidence for Tc-Ser acting upstream of these genes. Neverthess, it would be interesting to see if Tc-Ser could be a downstream target of these genes by analyzing the expression of Tc-Ser in Tc-cnc and Tc-croc RNAi background.

Although not involved in early patterning of the AMR, I was able to show that the growth of the labrum during embryogenesis is mediated by Notch signaling due to its control of cell proliferation. After loss of Tc-mibl function cell proliferation was significantly reduced in the labrum. A function for Notch signaling in coordinating cell proliferation events was also reported earlier in Drosophila and vertebrates in a different developmental context (Andersson et al., 2011; Estella et al., 2015; Hori et al., 2013). In Drosophila, mediation of cell proliferation upon Notch signaling is best reported in the eye (Baonza and Freeman, 2005; Chao et al., 2004; Domínguez and de Celis, 1998; Kenyon et al., 2003; Kumar and Moses, 2001; Reynolds-Kenneally and Mlodzik, 2005) and wing imaginal discs (de Celis and García-Bellido, 1994; Giraldez and Cohen, 2003; Go et al., 1998; Johnston and Edgar, 1998). Furthermore, it is also assumed to be necessary for the growth of the leg (Bishop et al., 1999; Celis et al., 1998; Rauskolb and Irvine, 1999).

However, the initial outgrowth of the labrum was not dependent on Notch signaling, although $T c$-Ser is already active in the embryo before the outgrowth is morphologically visible. In addition, cell proliferation was reduced but not abolished in RNAi animals. This could be explained in a situation where cell proliferation is also induced by other genes or by technical issues, e.g. that the knockdown is not complete. The most known upstream regulator of AMR $T c$-six3 was already proposed to play a role in proliferation in Tribolium (Kittelmann, 2012), because in Xenopus and zebrafish six3 is also connected to proliferation events (Del Bene et al., 2004; Gestri et al., 2005). Another potential candidate which could contribute in this growth event is the gene $T c-D l l$. Tc-Dll expression starts before outgrowth of the limbs and might contribute in growth control. To test this hypothesis cell proliferation assays should also be performed in Tc-Dll RNAi embryos. A cross-talk between Notch and other signaling pathways to regulate cell proliferation is also known in the Drosophila and vertebrates in 
some detail (Hurlbut et al., 2007). For example, Notch and Wg act synergistically to promote tissue growth in the wing imaginal disc of Drosophila (Giraldez and Cohen, 2003).

\subsubsection{Notch signaling has a distinct role in the appendage regulatory network in the labrum}

The evolution of the labrum is still enigmatic and debated. While some authors state that the labrum is a true appendage (Haas et al., 2001a; Kimm and Prpic, 2006; Popadíc et al., 1998), other argue that the labrum cannot be seen as a true homolog of the trunk appendages because of its divergent position, albeit it shows structural similarities (Posnien et al., 2009a). One explanation for the similarity of non homologous structures is based on co-option of gene regulatory networks (Moczek and Rose, 2009; Posnien et al., 2009a; Smith et al., 2014). If the labrum originated from appendages of a serial homolog of trunk segments, a similar function of genes in the patterning network of the trunk and labrum would be expected. However, I showed with respect to Notch signaling that the network is quite different, since Notch signaling acts earlier and more upstream of the leg pattering network.

Recent studies already demonstrated that a number of genes, which are involved in the appendage patterning network, show a similar expression in the labrum anlagen. This includes the genes Tc-homeothorax, Tc-BarH, Tc-bowl2, Tc-buttonhead, Tc-Dll, Tc-wg, Tc-dac and Tc-dpp (Beermann et al., 2001; Nagy and Carroll, 1994; Posnien et al., 2009a; Prpic et al., 2001; Schinko et al., 2008; van der Zee et al., 2006). Indeed, Tc-Ser is also expressed in a similar pattern in the labrum and the trunk appendages at late stages (Aranda et al., 2008). However, I showed that Tc-Ser expression starts much earlier in the AMR than in the legs. This data suggests that Tc-Ser has an earlier function in labrum development than in leg formation. In contrast to that, the expression domains of $T c-D l l, T c-d a c$ and $T c-w g$ in the labrum arise later in development.

It was predicted previously that a comparatively severe phenotype in the labrum in contrast to other appendages after $T c-D l l$ knockdown is an indication for a different role of $T c-D l l$ in the labrum (Schoppmeier and Damen, 2001; Smith et al., 2014). This was also true for the deletion of Tc-Ser and Tc-mibl in Tribolium, where the labrum was deleted while the legs were just shortened.

In this work, I also showed that disruption of the Notch signaling pathway abolished the expression domains of $T c-D a c$ and $T c-w g$. This was different in comparison to the leg where $T c-D a c$ and $T c-w g$ were unaffected upon Tc-mibl deletion. In addition, my data demonstrated that disruption of Notch signaling at least reduced the expression of $T c-D l l$ in the labrum and 
vice versa. This suggests that the Notch signaling pathway in the labrum is acting upstream in this network, while it is presumably acting downstream of the appendage patterning genes in the leg (Prpic and Damen, 2009; Rauskolb and Irvine, 1999). Moreover, the reduction of Tc$D l l$ in Tc-mibl RNAi background and vice versa indicated that these genes are interacting with each other in the most distal portion of the labrum but not the legs. In summary, the position of Notch signaling in the patterning network is different between legs and labrum.

However, it is difficult to determine whether the loss or reduction of the expression after knockdown of Tc-mibl is due to direct activation. Another explanation could be that the expression of these genes is reduced or lost due to missing tissue. Heat shock mediated ectopic expression of $\mathrm{Tc}$-Ser could shed light into this question. Additionally, ectopic expression of a cell cycle regulator like $C y c E$ could be an alternative to rescue the reduced cell proliferation phenotype in Tc-mibl RNAi animals. In Drosophila, Notch signaling was thought to act upstream of the Ey/Pax6 pathway and thereby promoting eye field identity (Kumar and Moses, 2001; Kurata et al., 2000). However, a distinct study demonstrated that loss of Notch function does not affect the selector gene expression. Here they could partially rescue both reduced growth of the eye disc and expression of Eyes absent (Eya) by ectopic expression of the cell cycle regulator Cyclin-E $(C y c E)$ which is a G1-S phase cyclin. Thus, the reduction in Eya expression is only a secondary consequence of reduced cell proliferation (Kenyon et al., 2003).

Another difference between labrum and leg is the timing of proliferation. I was able to show that Notch mediated cell proliferation is required for the proper outgrowth of the labrum. In contrast, a comparable early role for Notch in controlling cell proliferation at the beginning of the leg outgrowth excluded in this work.

However, my data did not exclude an involvement during later development. Indeed, it is likely that later in development Notch mediated cell proliferation might be important. The shortened leg phenotype after knockdown of Tc-mibl and Tc-Ser already indicated that not only joint formation but also growth of the appendages is disturbed.

This specific leg phenotype is similar to the one of other insects. In Drosophila, growth defects observed in Notch mutant legs are predicted to be a consequence of disturbed cell proliferation, but confirming data is still missing (Bishop et al., 1999; Rauskolb, 2001; Rauskolb and Irvine, 1999). Nevertheless, analysis of Notch and its ligand Ser in the spider cupiennius already demonstrated that knockdown of both genes results in reduced cell proliferation in the leg (Prpic and Damen, 2009). To test if Notch signaling plays also a role in 
regulation of cell proliferation in the leg, EdU proliferation assays should be performed at later stages of development in Tribolium.

Former investigations suggested another fundamental difference in the activation of $T c-D l l$ in the labrum than in ventral appendages. Here, It was shown that $T c-D l l$ expression depends on $T c-h h$ and $T c-w g$ function in all appendages but the labrum (Posnien et al., 2009a).

In summary, the function of Notch signaling in the appendage regulatory network and the labrum in Tribolium differs in four aspects. First, expression of Tc-Ser started in the AMR before it was detectable in the appendages. Second, the labrum phenotype after Tc-mibl and Tc-Ser RNAi is stronger in the labrum than it is in the legs. Third, Notch signaling in the labrum is acting more upstream of the appendage patterning network, while it is presumably acting downstream in the leg. Finally, Notch signaling led to reduction in proliferating cells during outgrowth of the labrum, while it plays no role in the initial outgrowth of the legs. Based on my results as well as on previous data, the gene regulatory network in the labrum reveals some major differences in the use of Notch signaling in the leg and the labrum (Figure $5.1)$.

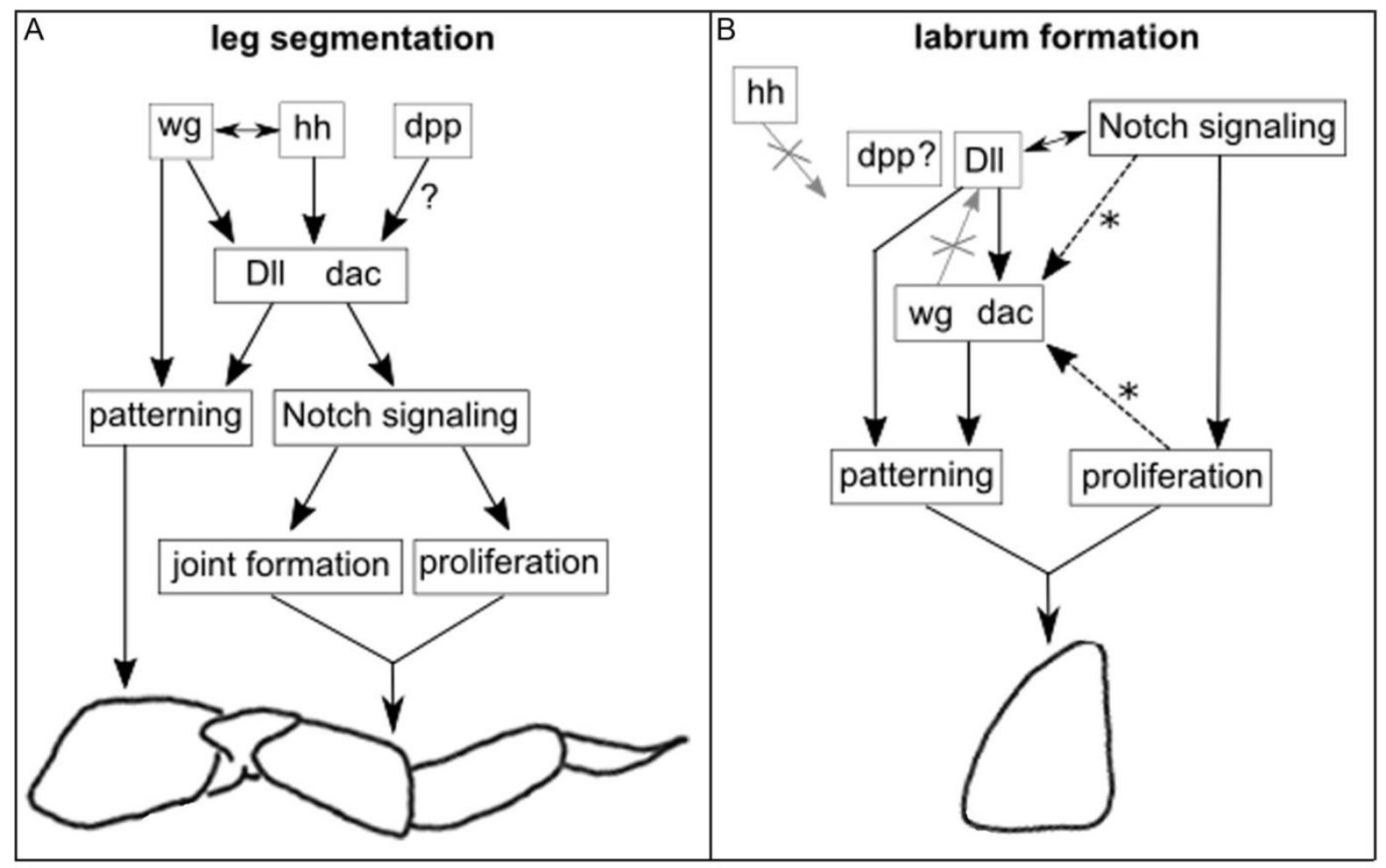

Figure 5.1: Comparison of the gene regulatory networks in the leg and the labrum of Tribolium - Arrows indicate activation. Asterisk indicate direct or indirect activation (A) $T c-w g$ function in leg allocation and distal and ventral leg patterning (Grossmann et al., 2009). Tc-wg regulation requires $T c$ - $h h$ activation in the leg, because over-activation of $h h$ signaling leads to over-expression of $T c-w g$ and down regulation of $T c-h h$ abolish expression of Tc-wg in the legs (Farzana and Brown, 2008; Posnien et al., 2009a). Additionally, expression of 
$T c-D l l$ is depended upon $T c-h h$ and $T c-w g$ function (Posnien et al., 2009a). The role of $T c-d p p$ in leg segmentation is still unclear (indicated by questionmark) (Ober and Jockusch, 2006; van der Zee et al., 2006). $T c-D l l$ in turn is a positive regulator of $T c-d a c$ (Prpic et al., 2001). Downstream of the patterning network, Notch signaling induces joint formation and growth of the leg (Angelini et al., 2012b; this work). (B) In contrast to the leg, expression of $T c-D l l$ in the labrum is independent of $T c-h h$ and $T c-w g$ function (Posnien et al., 2009a). A major role for Notch signaling is the induction of cell proliferation for early labrum growth. The Notch signaling pathway acts either directly or indirectly upstream of $T c$-dac and $T c$-wg (asterisk). In addition, Notch signaling and $T c$-Dll might interact with each other.

\subsection{Further Outlook}

Whether neurogenesis is affected in the head after disruption of Notch signaling was not tested in this work. In Drosophila, the ubiquitin ligase neur is a fly neurogenic gene, which activates Notch signaling via the ligand $\mathrm{Dl}$. Ser and mibl are only weakly neurogenic in Drosophila (Daskalaki et al., 2011; Lai and Rubin, 2001; Lai et al., 2005; Le Borgne et al., 2005b; Lehmann et al., 1983). However, mib is a neurogenic gene in vertebrates and was originally characterized in zebrafish for its role in lateral inhibition (Itoh et al., 2003). To test a neurogenic role in Tribolium Tc-mibl RNAi could be performed in a Tribolium brain imaging line (Koniszewski, 2011; Posnien et al., 2011b). Furthermore, in situ hybridization with early markers of neural specification could be done in Tc-mibl RNAi embryos, e.g. Tcachaete-scute or Tc-snail (Kux et al., 2013; Wheeler et al., 2003).

\subsection{The role of the GATA transcription factor $T c$-grn in labrum formation in Tribolium}

During this work, $T c$-grn was identified as a transcription factor gene contributing to labrum formation. I showed that expression of Tc-grn in Tribolium starts in the extraembryonic membranes in a few serosa cells and in the amnion. This expression could be connected to the head phenotype observed after Tc-grn RNAi. It was suggested previously, that the shape of the anterior head depends on morphogenetic movements including both the germ band and the extraembryonic tissues. While the serosa is probably not important for the proper development of the insect head (Kittelmann, 2012; van der Zee et al., 2005), amnion development and formation of the labrum are suggested to be connected. Knockdown of the gene Tc-tailup (tup) which is also expressed in the extraembryonic membranes, leads to deletion of the labrum. Moreover, the knockdown of members of these ush-group genes 
including tup leads to defects in head formation and dorsal closure of the scuttle fly Megaselia (Rafiqi et al., 2010). Thus, the role of Tc-grn in amnion development should be considered in future experiments.

As in Drosophila, Tc-grn expression is found in the procephalon in Tribolium where it marks the developing headlobes and in three separated domains, i.e. the labrum, the surrounding neurogenic tissue and in the ocular region in the head during later development (Lin et al., 1995). Loss of Tc-grn function led to a strong head phenotype where the labrum was missing and the head capsule was reduced in size. An abnormal head skeleton in grn mutants was also reported in Drosophila but detailed analyses were not performed (Brown and Castelli-Gair Hombría, 2000). Interestingly, my results indicated that Tc-grn was required rather for the proper morphogenetic movement of the labrum than pattern formation by regulating the expression of head patterning genes. Indeed, the expression of genes required for early AMR and labrum development (Tc-cnc and Tc-croc) were not affected in Tc-grn RNAi animals. However, this should be confirmed by staining further genes, which are also known to be required during early AMR and labrum development, in Tc-grn RNAi background. This includes, e.g. the genes six3 and Tc-tup (Kittelmann et al., 2013; Posnien et al., 2009a; Posnien et al., 2011b).

With respect to the appendage pattering network, my data gave evidence that Tc-grn could have a later role during development of the labrum by the activation of $T c-w g, T c-d a c$ and $T c$ Dll. In Tc-grn RNAi animals the expression domains of $T c-w g$ and $T c$-dac were abolished and the level of $T c-D l l$ expression reduced. In the appendages, these genes are known to be involved in the patterning process but the function during labrum development is still enigmatic. It remains to be studied, whether Tc-grn is activating these genes or whether the change in cell proliferation/morphogenesis secondarily lead to changes in expression patterns. Moreover, the observed loss of the labrum in Tc-grn RNAi first instar cuticles was not due to a lack of labrum anlagen during embryogenesis, which was shown by DAPI stainings. Apparently, the labrum anlagen were initiated normally and grew out as two separated labral buds. Interestingly, the labral buds fused precociously, e.g. during elongation of the appendages and not after the end of germ band retraction (Kimm and Prpic, 2006). Additionally, an abnormal rotation of the buds was observed. Tc-chx is normally expressed in the base of the labrum and in surrounding neurogenic tissue. In Tc-grn RNAi background, both expression domains of $T c$-chx were not affected in expression level but slightly shifted towards anterior. Thus, the results of my work suggest that Tc-grn could be required for proper cell movement during morphogenesis of the labrum. During morphogenesis, cells 
undergo different strategies, including cell movements by migration or rearrangement, shape, death and proliferation (Fristrom, 1988).

Indeed, head formation in Tribolium is accompanied by complex morphogenetic movements. The embryonic condensation is suggested to be accompanied by a variety of morphogenetic processes including cell rearrangement (Benton et al., 2013). Furthermore, the proper formation of the AMR requires the backfolding of anterior tissues. Here, initially anterior cells are displaced towards posterior (Kittelmann et al., 2013). Major morphogenetic movements are also required to transform the two dimensional head anlagen into a three dimensional head capsule (Posnien et al., 2010). It was demonstrated previously, that the loss of structures during embryogenesis (e.g. the loss of the stomodeum after Tc-croc RNAi) causes aberrant morphogenetic tissue movements which lead to misplacement of the labrum (Kittelmann et al., 2013). Additionally, cell movements are predicted to be an explanation for changes of the expression domains from the anterior tip of the germ band to a more posterior positions during later stages (Kittelmann, 2012).

An involvement of Tc-grn in such process is not unlikely, as a major role of $g r n$ in the morphogenetic mechanisms is already known for Drosophila. Here, grn is required for cell rearrangements of the posterior spiracles and the leg imaginal disc. The external structure of the spiracle called stigmatophore develops by cell rearrangement leading to protrusion of the structure. In grn mutant embryos the structure does not protrude because cells remaining in their position. On the other hand, ectopic expression of grn causes invagination of abdominal segments and the unsegmented telson (Brown and Castelli-Gair Hombría, 2000). In addition, these authors assumed that the changed shape of the legs in grn mutant cells could be linked to an alteration of cell rearrangement in the primordium.

In order to test the role of Tc-grn in morphogenesis of the labrum several tests could be performed. First, the morphology of Tc-grn RNAi embryos could be studied by FM® 1-43 staining throughout development. This could show when the tissue of the labrum is lost. This could be combined with fluorescent staining of $T c-\operatorname{ch} x$ or $T c-\operatorname{six} 3$ in combination with FM® 1-43 and DAPI. To further analyze the role of Tc-grn in labrum development, in vivo imaging experiments should be carried out both in wildtype and Tc-grn RNAi embryos. In the zebrafish in vivo imaging by scanned light sheet microscopy already gave comprehensive insights into rearrangement, division and migration of cells (Keller et al., 2008). In Tribolium live imaging was also already used successfully to record different cell and tissue dynamics during embryonic development (Benton et al., 2013; Kittelmann, 2012; Panfilio et al., 2013; Strobl and Stelzer, 2014). Additionally, ectopic expression could help to get a better insight 
into the function of Tc-grn in morphogenesis of the head. In Drosophila ectopic grn expression leads to defects in head involution (Brown and Castelli-Gair Hombría, 2000). In order to see if the labrum phenotype of Tc-grn RNAi could be also explained by aberrant cell proliferation or cell death, EdU proliferation assays and cleaved Drosophila Dcp-1 antibody staining should be performed, respectively. 


\section{References}

Abel, T., Michelson, A. M. and Maniatis, T. (1993). A Drosophila GATA family member that binds to Adh regulatory sequences is expressed in the developing fat body. Dev. Camb. Engl. 119, 623-633.

Abu-Shaar, M. and Mann, R. S. (1998). Generation of multiple antagonistic domains along the proximodistal axis during Drosophila leg development. Dev. Camb. Engl. 125, 3821-3830.

Abzhanov, A. and Kaufman, T. C. (1999). Homeotic genes and the arthropod head: Expression patterns of the labial, proboscipedia, and Deformed genes in crustaceans and insects. Proc. Natl. Acad. Sci. U. S. A. 96, 10224-10229.

Akam, M. (1998). Hox genes in arthropod development and evolution. Biol. Bull. 195, 373374.

Altschul, S. F., Gish, W., Miller, W., Myers, E. W. and Lipman, D. J. (1990). Basic local alignment search tool. J Mol Biol 215, 403-410.

Andersson, E. R., Sandberg, R. and Lendahl, U. (2011). Notch signaling: simplicity in design, versatility in function. Dev. Camb. Engl. 138, 3593-3612.

Angelini, D. R., Kikuchi, M. and Jockusch, E. L. (2009). Genetic patterning in the adult capitate antenna of the beetle Tribolium castaneum. Dev. Biol. 327, 240-251.

Angelini, D. R., Smith, F. W. and Jockusch, E. L. (2012a). Extent With Modification: Leg Patterning in the Beetle Tribolium castaneum and the Evolution of Serial Homologs. G3 Bethesda 2, 235-48.

Angelini, D. R., Smith, F. W., Aspiras, A. C., Kikuchi, M. and Jockusch, E. L. (2012b). Patterning of the Adult Mandibulate Mouthparts in the Red Flour Beetle, Tribolium castaneum. Genetics 190, 639-654.

Angelini, D. R., Smith, F. W. and Jockusch, E. L. (2012c). Extent With Modification: Leg Patterning in the Beetle Tribolium castaneum and the Evolution of Serial Homologs. G3 Bethesda Md 2, 235-248.

Angelini, D. R., Smith, F. W. and Jockusch, E. L. (2012d). Extent With Modification: Leg Patterning in the Beetle Tribolium castaneum and the Evolution of Serial Homologs. G3 GenesGenomesGenetics 2, 235-248.

Aranda, M. (2006). Functional analysis of a homolog of the pair-rule gene hairy in the shortgerm beetle Tribolium castaneum.

Aranda, M., Marques-Souza, H., Bayer, T. and Tautz, D. (2008). The role of the segmentation gene hairy in Tribolium. Dev. Genes Evol. 218, 465-477.

Arendt, D., Denes, A. S., Jekely, G. and Tessmar-Raible, K. (2008). The evolution of nervous system centralization. Philos Trans $R$ Soc Lond B Biol Sci 363, 1523-8.

Artavanis-Tsakonas, S., Rand, M. D. and Lake, R. J. (1999). Notch signaling: cell fate control and signal integration in development. Science 284, 770-776.

Axelrod, J. D., Matsuno, K., Artavanis-Tsakonas, S. and Perrimon, N. (1996). Interaction between Wingless and Notch signaling pathways mediated by dishevelled. Science 271, 1826-1832.

Baonza, A. and Freeman, M. (2005). Control of cell proliferation in the Drosophila eye by Notch signaling. Dev. Cell 8, 529-539.

Baumer, D., Strohlein, N. M. and Schoppmeier, M. (2012). Opposing effects of Notchsignaling in maintaining the proliferative state of follicle cells in the telotrophic ovary of the beetle Tribolium. Front Zool 9, 15. 
Beermann, A., Jay, D. G., Beeman, R. W., Hülskamp, M., Tautz, D. and Jürgens, G. (2001). The Short antennae gene of Tribolium is required for limb development and encodes the orthologue of the Drosophila Distal-less protein. Dev. Camb. Engl. 128, 287-297.

Beermann, A., Aranda, M. and Schröder, R. (2004). The Sp8 zinc-finger transcription factor is involved in allometric growth of the limbs in the beetle Tribolium castaneum. Development 131, 733-42.

Benton, M. A., Akam, M. and Pavlopoulos, A. (2013). Cell and tissue dynamics during Tribolium embryogenesis revealed by versatile fluorescence labeling approaches. Dev. Camb. Engl. 140, 3210-3220.

Berghammer, A. J., Klingler, M. and Wimmer, E. A. (1999). A universal marker for transgenic insects. Nature 402, 370-1.

Berghammer, A. J., Weber, M., Trauner, J. and Klingler, M. (2009). Red flour beetle (Tribolium) germline transformation and insertional mutagenesis. Cold Spring Harb. Protoc. 2009, pdb.prot5259.

Bishop, S. A., Klein, T., Arias, A. M. and Couso, J. P. (1999). Composite signalling from Serrate and Delta establishes leg segments in Drosophila through Notch. Dev. Camb. Engl. 126, 2993-3003.

Bonifacino, J. S. and Weissman, A. M. (2002). Ubiquitin and the Control of Protein Fate in the Secretory and Endocytic Pathways.

Borggrefe, T. and Oswald, F. (2009). The Notch signaling pathway: transcriptional regulation at Notch target genes. Cell. Mol. Life Sci. CMLS 66, 1631-1646.

Boulianne, G. L., de la Concha, A., Campos-Ortega, J. A., Jan, L. Y. and Jan, Y. N. (1991). The Drosophila neurogenic gene neuralized encodes a novel protein and is expressed in precursors of larval and adult neurons. EMBO J. 10, 2975-2983.

Bray, S. J. (2006). Notch signalling: a simple pathway becomes complex. Nat. Rev. Mol. Cell Biol. 7, 678-689.

Brook, W. J., Diaz-Benjumea, F. J. and Cohen, S. M. (1996). Organizing Spatial Pattern in Limb Development. Annu. Rev. Cell Dev. Biol. 12, 161-180.

Brown, S. and Castelli-Gair Hombría, J. (2000). Drosophila grain encodes a GATA transcription factor required for cell rearrangement during morphogenesis. Dev. Camb. Engl. 127, 4867-4876.

Brown, S. J., Mahaffey, J. P., Lorenzen, M. D., Denell, R. E. and Mahaffey, J. W. (1999). Using RNAi to investigate orthologous homeotic gene function during development of distantly related insects. Evol Dev 1, 11-5.

Brown, S., Fellers, J., Shippy, T., Denell, R., Stauber, M. and Schmidt-Ott, U. (2001). A strategy for mapping bicoid on the phylogenetic tree. Curr Biol 11, R43-4.

Brown, S. J., Shippy, T. D., Miller, S., Bolognesi, R., Beeman, R. W., Lorenzen, M. D., Bucher, G., Wimmer, E. A. and Klingler, M. (2009). The red flour beetle, Tribolium castaneum (Coleoptera): a model for studies of development and pest biology. Cold Spring Harb Protoc 2009, pdb emo126.

Bucher, G. and Klingler, M. (2004). Divergent segmentation mechanism in the short germ insect Tribolium revealed by giant expression and function. Development 131, 172940.

Bucher, G. and Klingler, M. (2005). Tribolium mae expression suggests roles in terminal and midline patterning and in the specification of mesoderm. Dev Genes Evol 215, 478-81.

Bucher, G. and Wimmer, E. A. (2005). Beetle a-head. BIF Futura 20, 164-169.

Bucher, G., Scholten, J. and Klingler, M. (2002). Parental RNAi in Tribolium (Coleoptera). Curr Biol 12, R85-6. 
Bucher, G., Farzana, L., Brown, S. J. and Klingler, M. (2005). Anterior localization of maternal mRNAs in a short germ insect lacking bicoid. Evol Dev 7, 142-9.

Budd, G. E. (2002). A palaeontological solution to the arthropod head problem. Nature 417, 271-5.

Cabrera, C. V. (1990). Lateral inhibition and cell fate during neurogenesis in Drosophila: the interactions between scute, Notch and Delta. Dev. Camb. Engl. 110, 733-742.

Celis, J. F. de, Tyler, D. M., Celis, J. de and Bray, S. J. (1998). Notch signalling mediates segmentation of the Drosophila leg. Development 125, 4617-4626.

Cerny, A. C., Grossmann, D., Bucher, G. and Klingler, M. (2008). The Tribolium ortholog of knirps and knirps-related is crucial for head segmentation but plays a minor role during abdominal patterning. Dev. Biol. 321, 284-294.

Chao, J.-L., Tsai, Y.-C., Chiu, S.-J. and Sun, Y. H. (2004). Localized Notch signal acts through eyg and upd to promote global growth in Drosophila eye. Development 131, 3839-3847.

Chen, W. and Casey Corliss, D. (2004). Three modules of zebrafish Mind bomb work cooperatively to promote Delta ubiquitination and endocytosis. Dev. Biol. 267, 361373.

Cohen, S. M. and Jürgens, G. (1990). Mediation of Drosophila head development by gaplike segmentation genes. Nature 346, 482-5.

Cohen, S. and Jürgens, G. (1991). Drosophila headlines. Trends Genet 7, 267-72.

Coulcher, J. F. and Telford, M. J. (2012). Cap'n'collar differentiates the mandible from the maxilla in the beetle Tribolium castaneum. Evodevo 3, 25.

Crozatier, M., Valle, D., Dubois, L., Ibnsouda, S. and Vincent, A. (1999). Head versus trunk patterning in the Drosophila embryo; collier requirement for formation of the intercalary segment. Development 126, 4385-94.

Daskalaki, A., Shalaby, N. A., Kux, K., Tsoumpekos, G., Tsibidis, G. D., Muskavitch, M. A. T. and Delidakis, C. (2011). Distinct intracellular motifs of Delta mediate its ubiquitylation and activation by Mindbomb1 and Neuralized. J. Cell Biol. 195, 10171031.

Daubresse, G., Deuring, R., Moore, L., Papoulas, O., Zakrajsek, I., Waldrip, W. R., Scott, M. P., Kennison, J. A. and Tamkun, J. W. (1999). The Drosophila kismet gene is related to chromatin-remodeling factors and is required for both segmentation and segment identity. Development 126, 1175-87.

Davis, G. K. and Patel, N. H. (2002). SHORT, LONG, AND BEYOND: Molecular and Embryological Approaches to Insect Segmentation. Annu. Rev. Entomol. 47, 669-699.

De Celis, J. F. and García-Bellido, A. (1994). Roles of the Notch gene in Drosophila wing morphogenesis. Mech. Dev. 46, 109-122.

Del Bene, F., Tessmar-Raible, K. and Wittbrodt, J. (2004). Direct interaction of geminin and Six3 in eye development. Nature 427, 745-9.

Diaz-Benjumea, F. J. and Cohen, S. M. (1995). Serrate signals through Notch to establish a Wingless-dependent organizer at the dorsal/ventral compartment boundary of the Drosophila wing. Dev. Camb. Engl. 121, 4215-4225.

Diederich, R. J., Merrill, V. K., Pultz, M. A. and Kaufman, T. C. (1989). Isolation, structure, and expression of labial, a homeotic gene of the Antennapedia Complex involved in Drosophila head development. Genes Dev 3, 399-414.

Diederich, R. J., Pattatucci, A. M. and Kaufman, T. C. (1991). Developmental and evolutionary implications of labial, Deformed and engrailed expression in the Drosophila head. Development 113, 273-81.

Domínguez, M. and de Celis, J. F. (1998). A dorsal/ventral boundary established by Notch controls growth and polarity in the Drosophila eye. Nature 396, 276-278. 
Dönitz, J., Grossmann, D., Schild, I., Schmitt-Engel, C., Bradler, S., Prpic, N.-M. and Bucher, G. (2013). TrOn: An Anatomical Ontology for the Beetle Tribolium castaneum. PLOS ONE 8, e70695.

Dönitz, J., Schmitt-Engel, C., Grossmann, D., Gerischer, L., Tech, M., Schoppmeier, M., Klingler, M. and Bucher, G. (2014). iBeetle-Base: a database for RNAi phenotypes in the red flour beetle Tribolium castaneum. Nucleic Acids Res. gku1054.

Dönitz, J., Schmitt-Engel, C., Grossmann, D., Gerischer, L., Tech, M., Schoppmeier, M., Klingler, M. and Bucher, G. (2015). iBeetle-Base: a database for RNAi phenotypes in the red flour beetle Tribolium castaneum. Nucleic Acids Res. 43, D720-D725.

Duncan, D. M., Burgess, E. A. and Duncan, I. (1998). Control of distal antennal identity and tarsal development in Drosophila by spineless-aristapedia, a homolog of the mammalian dioxin receptor. Genes Dev. 12, 1290-1303.

Economou, A. D. and Telford, M. J. (2009). Comparative gene expression in the heads of Drosophila melanogaster and Tribolium castaneum and the segmental affinity of the Drosophila hypopharyngeal lobes. Evol Dev 11, 88-96.

Eernisse, D. J., Albert, J. S. and Anderson, F. E. (1992). Annelida and Arthropoda are Not Sister Taxa: A Phylogenetic Analysis of Spiralian Metazoan Morphology. Syst. Biol. 41, 305-330.

Estella, C., Baonza, A. and Departamento de Biología Molecular and Centro de Biología Molecular SeveroOchoa, Universidad Autónoma de Madrid (UAM), Madrid, Spain;Departamento de Biología Molecular and Centro de Biología Molecular SeveroOchoa, Universidad Autónoma de Madrid (UAM), Madrid, Spain (2015). Cell proliferation control by Notch signalling during imaginal discs development in Drosophila. AIMS Genet. 2, 70-96.

Farzana, L. and Brown, S. J. (2008). Hedgehog signaling pathway function conserved in Tribolium segmentation. Dev. Genes Evol. 218, 181-192.

Finkelstein, R. and Perrimon, N. (1991). The molecular genetics of head development in Drosophila melanogaster. Development 112, 899-912.

Fleming, R. J. (1998). Structural conservation of Notch receptors and ligands. Semin. Cell Dev. Biol. 9, 599-607.

Fleming, R. J., Scottgale, T. N., Diederich, R. J. and Artavanis-Tsakonas, S. (1990). The gene Serrate encodes a putative EGF-like transmembrane protein essential for proper ectodermal development in Drosophila melanogaster. Genes Dev. 4, 2188-2201.

Fortini, M. E. (2002). Gamma-secretase-mediated proteolysis in cell-surface-receptor signalling. Nat. Rev. Mol. Cell Biol. 3, 673-684.

Fristrom, D. (1988). The cellular basis of epithelial morphogenesis. A review. Tissue Cell 20, 645-690.

Fryer, C. J., White, J. B. and Jones, K. A. (2004). Mastermind Recruits CycC:CDK8 to Phosphorylate the Notch ICD and Coordinate Activation with Turnover. Mol. Cell 16, 509-520.

Fu, J., Posnien, N., Bolognesi, R., Fischer, T. D., Rayl, P., Oberhofer, G., Kitzmann, P., Brown, S. J. and Bucher, G. (2012). Asymmetrically expressed axin required for anterior development in Tribolium. Proc. Natl. Acad. Sci. 109, 7782-7786.

Garces, A. and Thor, S. (2006). Specification of Drosophila aCC motoneuron identity by a genetic cascade involving even-skipped, grain and zfh1. Dev. Camb. Engl. 133, 14451455.

Gestri, G., Carl, M., Appolloni, I., Wilson, S. W., Barsacchi, G. and Andreazzoli, M. (2005). Six3 functions in anterior neural plate specification by promoting cell proliferation and inhibiting Bmp4 expression. Development 132, 2401-13. 
Giraldez, A. J. and Cohen, S. M. (2003). Wingless and Notch signaling provide cell survival cues and control cell proliferation during wing development. Dev. Camb. Engl. 130, 6533-6543.

Glittenberg, M., Pitsouli, C., Garvey, C., Delidakis, C. and Bray, S. (2006). Role of conserved intracellular motifs in Serrate signalling, cis-inhibition and endocytosis. EMBO J. 25, 4697-4706.

Go, M. J., Eastman, D. S. and Artavanis-Tsakonas, S. (1998). Cell proliferation control by Notch signaling in Drosophila development. Dev. Camb. Engl. 125, 2031-2040.

Godt, D., Couderc, J. L., Cramton, S. E. and Laski, F. A. (1993). Pattern formation in the limbs of Drosophila: bric a brac is expressed in both a gradient and a wave-like pattern and is required for specification and proper segmentation of the tarsus. Development 119, 799-812.

Grossmann, D., Scholten, J. and Prpic, N.-M. (2009). Separable functions of wingless in distal and ventral patterning of the Tribolium leg. Dev. Genes Evol. 219, 469-479.

Grossniklaus, U., Pearson, R. K. and Gehring, W. J. (1992). The Drosophila sloppy paired locus encodes two proteins involved in segmentation that show homology to mammalian transcription factors. Genes Dev 6, 1030-51.

Grossniklaus, U., Cadigan, K. M. and Gehring, W. J. (1994). Three maternal coordinate systems cooperate in the patterning of the Drosophila head. Development 120, 315571.

Haas, M. S., Brown, S. J. and Beeman, R. W. (2001a). Homeotic evidence for the appendicular origin of the labrum in Tribolium castaneum. Dev Genes Evol 211, 96102.

Haas, M. S., Brown, S. J. and Beeman, R. W. (2001b). Pondering the procephalon: the segmental origin of the labrum. Dev Genes Evol 211, 89-95.

Heitzler, P., Haenlin, M., Ramain, P., Calleja, M. and Simpson, P. (1996). A genetic analysis of pannier, a gene necessary for viability of dorsal tissues and bristle positioning in Drosophila. Genetics 143, 1271-1286.

Hicke, L. (2001). Protein regulation by monoubiquitin. Nat. Rev. Mol. Cell Biol. 2, 195-201.

Hing, H. K., Sun, X. and Artavanis-Tsakonas, S. (1994). Modulation of wingless signaling by Notch in Drosophila. Mech. Dev. 47, 261-268.

Hori, K., Sen, A. and Artavanis-Tsakonas, S. (2013). Notch signaling at a glance. J. Cell Sci. 126, 2135-2140.

Horn, T. and Boutros, M. (2010). E-RNAi: a web application for the multi-species design of RNAi reagents--2010 update. Nucleic Acids Res 38, W332-9.

Hunnekuhl, V. S. and Akam, M. (2014). An anterior medial cell population with an apicalorgan-like transcriptional profile that pioneers the central nervous system in the centipede Strigamia maritima. Dev. Biol. 396, 136-149.

Hurlbut, G. D., Kankel, M. W., Lake, R. J. and Artavanis-Tsakonas, S. (2007). Crossing paths with Notch in the hyper-network. Curr. Opin. Cell Biol. 19, 166-175.

Ingham, P. W. (1988). The molecular genetics of embryonic pattern formation in Drosophila. Nature 335, 25-34.

Itoh, M., Kim, C.-H., Palardy, G., Oda, T., Jiang, Y.-J., Maust, D., Yeo, S.-Y., Lorick, K., Wright, G. J., Ariza-McNaughton, L., et al. (2003). Mind bomb is a ubiquitin ligase that is essential for efficient activation of Notch signaling by Delta. Dev. Cell 4, $67-82$.

Johnston, L. A. and Edgar, B. A. (1998). Wingless and Notch regulate cell-cycle arrest in the developing Drosophila wing. Nature 394, 82-84.

Jürgens, G., Lehmann, R., Schardin, M. and Nüsslein-Volhard, C. (1986). Segmental organisation of the head in the embryo of Drosophila melanogaster. Roux's Arch Dev Biol 195, 359-377. 
Keller, P. J., Schmidt, A. D., Wittbrodt, J. and Stelzer, E. H. K. (2008). Reconstruction of Zebrafish Early Embryonic Development by Scanned Light Sheet Microscopy. Science 322, 1065-1069.

Kenyon, K. L., Ranade, S. S., Curtiss, J., Mlodzik, M. and Pignoni, F. (2003). Coordinating proliferation and tissue specification to promote regional identity in the Drosophila head. Dev. Cell 5, 403-414.

Kidd, S., Kelley, M. R. and Young, M. W. (1986). Sequence of the notch locus of Drosophila melanogaster: relationship of the encoded protein to mammalian clotting and growth factors. Mol. Cell. Biol. 6, 3094-3108.

Kim, H. S., Murphy, T., Xia, J., Caragea, D., Park, Y., Beeman, R. W., Lorenzen, M. D., Butcher, S., Manak, J. R. and Brown, S. J. (2010). BeetleBase in 2010: revisions to provide comprehensive genomic information for Tribolium castaneum. Nucleic Acids Res. 38, D437-442.

Kimm, M. A. and Prpic, N. M. (2006). Formation of the arthropod labrum by fusion of paired and rotated limb-bud-like primordia. Zoomorphology 125, 147 - 155 .

Kittelmann, S. (2012). Formation of the clypeolabral region during embryonic head development of the red flour beetle Tribolium castaneum. $\mathrm{PhD}$ thesis.

Kittelmann, S., Ulrich, J., Posnien, N. and Bucher, G. (2013). Changes in anterior head patterning underlie the evolution of long germ embryogenesis. Dev. Biol. 374, 174 184.

Kitzmann, P., Schwirz, J., Schmitt-Engel, C. and Bucher, G. (2013). RNAi phenotypes are influenced by the genetic background of the injected strain. BMC Genomics 14, 5.

Klingler, M. (2004). Tribolium. Curr. Biol. 14, R639-R640.

Klueg, K. M. and Muskavitch, M. A. (1999). Ligand-receptor interactions and transendocytosis of Delta, Serrate and Notch: members of the Notch signalling pathway in Drosophila. J. Cell Sci. 112 ( Pt 19), 3289-3297.

Koniszewski, N. (2011). Functional analysis of embryonic brain development in Tribolium castaneum.

Kooh, P. J., Fehon, R. G. and Muskavitch, M. A. (1993). Implications of dynamic patterns of Delta and Notch expression for cellular interactions during Drosophila development. Dev. Camb. Engl. 117, 493-507.

Kumar, J. P. and Moses, K. (2001). EGF receptor and Notch signaling act upstream of Eyeless/Pax6 to control eye specification. Cell 104, 687-697.

Kurata, S., Go, M. J., Artavanis-Tsakonas, S. and Gehring, W. J. (2000). Notch signaling and the determination of appendage identity. Proc Natl Acad Sci U A 97, 2117-22.

Kux, K., Kiparaki, M. and Delidakis, C. (2013). The two Tribolium E(spl) genes show evolutionarily conserved expression and function during embryonic neurogenesis. Mech. Dev. 130, 207-225.

Lai, E. C. (2004). Notch signaling: control of cell communication and cell fate. Dev. Camb. Engl. 131, 965-973.

Lai, E. C. and Rubin, G. M. (2001). neuralized functions cell-autonomously to regulate a subset of notch-dependent processes during adult Drosophila development. Dev. Biol. 231, 217-233.

Lai, E. C., Roegiers, F., Qin, X., Jan, Y. N. and Rubin, G. M. (2005). The ubiquitin ligase Drosophila Mind bomb promotes Notch signaling by regulating the localization and activity of Serrate and Delta. Dev. Camb. Engl. 132, 2319-2332.

Le Borgne, R. and Schweisguth, F. (2003a). Notch Signaling: Endocytosis Makes Delta Signal Better. Curr. Biol. 13, R273-R275.

Le Borgne, R. and Schweisguth, F. (2003b). Unequal segregation of Neuralized biases Notch activation during asymmetric cell division. Dev. Cell 5, 139-148. 
Le Borgne, R., Bardin, A. and Schweisguth, F. (2005a). The roles of receptor and ligand endocytosis in regulating Notch signaling. Dev. Camb. Engl. 132, 1751-1762.

Le Borgne, R., Remaud, S., Hamel, S. and Schweisguth, F. (2005b). Two distinct E3 ubiquitin ligases have complementary functions in the regulation of delta and serrate signaling in Drosophila. PLoS Biol. 3, e96.

Lecuit, T. and Cohen, S. M. (1997). Proximal-distal axis formation in the Drosophila leg. Nature 388, 139-145.

Lehmann, R., Jiménez, F., Dietrich, U. and Campos-Ortega, J. A. (1983). On the phenotype and development of mutants of early neurogenesis inDrosophila melanogaster. Wilhelm Rouxs Arch. Dev. Biol. 192, 62-74.

Lemke, S., Stauber, M., Shaw, P. J., Rafiqi, A. M., Prell, A. and Schmidt-Ott, U. (2008). Bicoid occurrence and Bicoid-dependent hunchback regulation in lower cyclorrhaphan flies. Evol Dev 10, 413-20.

Lewis, E. B. (1978). A gene complex controlling segmentation in Drosophila. Nature 276, $565-570$.

Lin, W.-H., Huang, L.-H., Yeh, J.-Y., Hoheisel, J., Lehrach, H., Sun, Y. H. and Tsai, S.F. (1995). Expression of a Drosophila GATA Transcription Factor in Multiple Tissues in the Developing Embryos IDENTIFICATION OF HOMOZYGOUS LETHAL MUTANTS WITH P-ELEMENT INSERTION AT THE PROMOTER REGION. $J$. Biol. Chem. 270, 25150-25158.

Lowe, C. J., Wu, M., Salic, A., Evans, L., Lander, E., Stange-Thomann, N., Gruber, C. E., Gerhart, J. and Kirschner, M. (2003). Anteroposterior patterning in hemichordates and the origins of the chordate nervous system. Cell 113, 853-65.

Martín-Bermudo, M. D., Carmena, A. and Jiménez, F. (1995). Neurogenic genes control gene expression at the transcriptional level in early neurogenesis and in mesectoderm specification. Dev. Camb. Engl. 121, 219-224.

McQuilton, P., St Pierre, S. E., Thurmond, J. and FlyBase Consortium (2012). FlyBase 101--the basics of navigating FlyBase. Nucleic Acids Res. 40, D706-714.

Mehlhorn, H. (1995). Grundriß der Zoologie. 2nd ed. Stuttgart: Gustav Fischer Verlag.

Merrill, V. K., Diederich, R. J., Turner, F. R. and Kaufman, T. C. (1989). A genetic and developmental analysis of mutations in labial, a gene necessary for proper head formation in Drosophila melanogaster. Dev. Biol. 135, 376-391.

Mito, T., Shinmyo, Y., Kurita, K., Nakamura, T., Ohuchi, H. and Noji, S. (2011). Ancestral functions of Delta/Notch signaling in the formation of body and leg segments in the cricket Gryllus bimaculatus. Dev. Camb. Engl. 138, 3823-3833.

Moczek, A. P. and Rose, D. J. (2009). Differential recruitment of limb patterning genes during development and diversification of beetle horns. Proc Natl Acad Sci U A.

Mumm, J. S. and Kopan, R. (2000). Notch Signaling: From the Outside In. Dev. Biol. 228, $151-165$.

Nagy, L. M. and Carroll, S. (1994). Conservation of wingless patterning functions in the short-germ embryos of Tribolium castaneum. Nature 367, 460-463.

Nüsslein-Volhard, C. and Wieschaus, E. (1980). Mutations affecting segment number and polarity in Drosophila. Nature 287, 795-801.

Ober, K. A. and Jockusch, E. L. (2006). The roles of wingless and decapentaplegic in axis and appendage development in the red flour beetle, Tribolium castaneum. Dev Biol 294, 391-405.

Oberhofer, G., Grossmann, D., Siemanowski, J. L., Beissbarth, T. and Bucher, G. (2014). Wnt/ $\beta$-catenin signaling integrates patterning and metabolism of the insect growth zone. Development dev.112797. 
Pan, D. and Rubin, G. M. (1997). Kuzbanian controls proteolytic processing of Notch and mediates lateral inhibition during Drosophila and vertebrate neurogenesis. Cell 90, 271-280.

Panfilio, K. A., Oberhofer, G. and Roth, S. (2013). High plasticity in epithelial morphogenesis during insect dorsal closure. Biol. Open 2, 1108-1118.

Parks, A. L., Klueg, K. M., Stout, J. R. and Muskavitch, M. A. (2000). Ligand endocytosis drives receptor dissociation and activation in the Notch pathway. Dev. Camb. Engl. 127, 1373-1385.

Popadíc, A., Panganiban, G., Rusch, D., Shear, W. A. and Kaufman, T. C. (1998). Molecular evidence for the gnathobasic derivation of arthropod mandibles and for the appendicular origin of the labrum and other structures. Dev. Genes Evol. 208, 142150.

Posnien, N. and Bucher, G. (2010). Formation of the insect head involves lateral contribution of the intercalary segment, which depends on Tc-labial function. Dev Biol 338, 107-16.

Posnien, N., Bashasab, F. and Bucher, G. (2009a). The insect upper lip (labrum) is a nonsegmental appendage-like structure. Evol Dev 11, 479-487.

Posnien, N., Schinko, J., Grossmann, D., Shippy, T. D., Konopova, B. and Bucher, G. (2009b). RNAi in the red flour beetle (Tribolium). Cold Spring Harb. Protoc. 2009, pdb.prot5256.

Posnien, N., Schinko, J. B., Kittelmann, S. and Bucher, G. (2010). Genetics, development and composition of the insect head - A beetle's view. Arthropod Struct Dev 39, 399410.

Posnien, N., Koniszewski, N. and Bucher, G. (2011a). Insect Tc-six4 marks a unit with similarity to vertebrate placodes. Dev Biol 350, 208-216.

Posnien, N., Koniszewski, N. D. B., Hein, H. J. and Bucher, G. (2011b). Candidate Gene Screen in the Red Flour Beetle Tribolium Reveals Six3 as Ancient Regulator of Anterior Median Head and Central Complex Development. PLoS Genet. 7, e1002418.

Prpic, N. M. and Damen, W. G. (2009). Notch-mediated segmentation of the appendages is a molecular phylotypic trait of the arthropods. Dev Biol 326, 262-71.

Prpic, N. M., Wigand, B., Damen, W. G. and Klingler, M. (2001). Expression of dachshund in wild-type and Distal-less mutant Tribolium corroborates serial homologies in insect appendages. Dev. Genes Evol. 211, 467-477.

Rafiqi, A. M., Lemke, S. and Schmidt-Ott, U. (2010). Postgastrular zen expression is required to develop distinct amniotic and serosal epithelia in the scuttle fly Megaselia. Dev. Biol. 341, 282-290.

Ramain, P., Heitzler, P., Haenlin, M. and Simpson, P. (1993). pannier, a negative regulator of achaete and scute in Drosophila, encodes a zinc finger protein with homology to the vertebrate transcription factor GATA-1. Development 119, 1277-1291.

Rauskolb, C. (2001). The establishment of segmentation in the Drosophila leg. Dev. Camb. Engl. 128, 4511-4521.

Rauskolb, C. and Irvine, K. D. (1999). Notch-mediated segmentation and growth control of the Drosophila leg. Dev Biol 210, 339-50.

Rebay, I., Fleming, R. J., Fehon, R. G., Cherbas, L., Cherbas, P. and ArtavanisTsakonas, S. (1991). Specific EGF repeats of Notch mediate interactions with Delta and serrate: Implications for notch as a multifunctional receptor. Cell 67, 687-699.

Rehorn, K. P., Thelen, H., Michelson, A. M. and Reuter, R. (1996). A molecular aspect of hematopoiesis and endoderm development common to vertebrates and Drosophila. Dev. Camb. Engl. 122, 4023-4031.

Reichert, H. (2009). Evolutionary conservation of mechanisms for neural regionalization, proliferation and interconnection in brain development. Biol Lett 5, 112-6. 
Reichert, H. and Simeone, A. (2001). Developmental genetic evidence for a monophyletic origin of the bilaterian brain. Philos Trans R Soc Lond B Biol Sci 356, 1533-44.

Reuter, R. (1994). The gene serpent has homeotic properties and specifies endoderm versus ectoderm within the Drosophila gut. Dev. Camb. Engl. 120, 1123-1135.

Reynolds-Kenneally, J. and Mlodzik, M. (2005). Notch signaling controls proliferation through cell-autonomous and non-autonomous mechanisms in the Drosophila eye. Dev. Biol. 285, 38-48.

Richards, S., Gibbs, R. A., Weinstock, G. M., Brown, S. J., Denell, R., Beeman, R. W., Gibbs, R., Beeman, R. W., Brown, S. J., Bucher, G., et al. (2008). The genome of the model beetle and pest Tribolium castaneum. Nature 452, 949-55.

Rogers, B. T. and Kaufman, T. C. (1996). Structure of the insect head as revealed by the EN protein pattern in developing embryos. Dev. Camb. Engl. 122, 3419-3432.

Rogers, B. T. and Kaufman, T. C. (1997). Structure of the insect head in ontogeny and phylogeny: a view from Drosophila. Int Rev Cytol 174, 1-84.

Rostovtsev, V. V., Green, L. G., Fokin, V. V. and Sharpless, K. B. (2002). A stepwise huisgen cycloaddition process: copper(I)-catalyzed regioselective "ligation" of azides and terminal alkynes. Angew. Chem. Int. Ed Engl. 41, 2596-2599.

Saitou, N. and Nei, M. (1987). The neighbor-joining method: a new method for reconstructing phylogenetic trees. Mol Biol Evol 4, 406-25.

Salic, A. and Mitchison, T. J. (2008). A chemical method for fast and sensitive detection of DNA synthesis in vivo. Proc. Natl. Acad. Sci. U. S. A. 105, 2415-2420.

Sambrook, J. and Russel, D. W. (2001). Molecular Cloning: A Laboratory Manual. 3rd ed. New York: Cold Spring Harbor Laboratory.

Sanchez-Salazar, J., Pletcher, M. T., Bennett, R. L., Brown, S. J., Dandamudi, T. J., Denell, R. E. and Doctor, J. S. (1996). The Tribolium decapentaplegic gene is similar in sequence, structure, and expression to the Drosophila dpp gene. Dev. Genes Evol. 206, 237-246.

Sandmann, T., Jakobsen, J. S. and Furlong, E. E. (2006). ChIP-on-chip protocol for genome-wide analysis of transcription factor binding in Drosophila melanogaster embryos. Nat Protoc 1, 2839-55.

Sarrazin, A. F., Peel, A. D. and Averof, M. (2012). A Segmentation Clock with TwoSegment Periodicity in Insects. Science.

Schaeper, N. D., Pechmann, M., Damen, W. G., Prpic, N. M. and Wimmer, E. A. (2010). Evolutionary plasticity of collier function in head development of diverse arthropods. Dev Biol 344, 363-76.

Schinko, J. B., Kreuzer, N., Offen, N., Posnien, N., Wimmer, E. A. and Bucher, G. (2008). Divergent functions of orthodenticle, empty spiracles and buttonhead in early head patterning of the beetle Tribolium castaneum (Coleoptera). Dev Biol 317, 60013.

Schinko, J., Posnien, N., Kittelmann, S., Koniszewski, N. and Bucher, G. (2009). Single and Double Whole-Mount In Situ Hybridization in Red Flour Beetle (Tribolium) Embryos. Cold Spring Harb. Protoc. 2009, pdb.prot5258-.

Schinko, J. B., Weber, M., Viktorinova, I., Kiupakis, A., Averof, M., Klingler, M., Wimmer, E. A. and Bucher, G. (2010). Functionality of the GAL4/UAS system in Tribolium requires the use of endogenous core promoters. BMC Dev Biol 10, 53.

Schinko, J., Hillebrand, K. and Bucher, G. (2012). Heat shock-mediated misexpression of genes in the beetle Tribolium castaneum. Dev Genes Evol Dev Genes Evol., 287-98.

Schmidt-Ott, U. and Technau, G. M. (1992). Expression of en and wg in the embryonic head and brain of Drosophila indicates a refolded band of seven segment remnants. Development 116, 111-25. 
Schmidt-Ott, U. and Technau, G. M. (1994). Fate-mapping in the procephalic region of the embryonic Drosophila head. Rouxs Arch Dev Biol 203, 367-373.

Schmidt-Ott, U., Gonzalez-Gaitan, M., Jackle, H. and Technau, G. M. (1994). Number, identity, and sequence of the Drosophila head segments as revealed by neural elements and their deletion patterns in mutants. Proc Natl Acad Sci U A 91, 8363-7.

Schmucker, D. and Chen, B. (2009). Dscam and DSCAM: complex genes in simple animals, complex animals yet simple genes. Genes Dev 23, 147-56.

Schmucker, D., Clemens, J. C., Shu, H., Worby, C. A., Xiao, J., Muda, M., Dixon, J. E. and Zipursky, S. L. (2000). Drosophila Dscam is an axon guidance receptor exhibiting extraordinary molecular diversity. Cell 101, 671-84.

Schneider, C. A., Rasband, W. S. and Eliceiri, K. W. (2012). NIH Image to ImageJ: 25 years of image analysis. Nat. Methods 9, 671-675.

Scholtz, G. and Edgecombe, G. D. (2006). The evolution of arthropod heads: reconciling morphological, developmental and palaeontological evidence. Dev Genes Evol 216, 395-415.

Schoppmeier, M. and Damen, W. G. (2001). Double-stranded RNA interference in the spider Cupiennius salei: the role of Distal-less is evolutionarily conserved in arthropod appendage formation. Dev. Genes Evol. 211, 76-82.

Schoppmeier, M. and Schröder, R. (2005). Maternal torso signaling controls body axis elongation in a short germ insect. Curr Biol 15, 2131-6.

Schröder, R. (2003). The genes orthodenticle and hunchback substitute for bicoid in the beetle Tribolium. Nature 422, 621-5.

Schröder, R., Eckert, C., Wolff, C. and Tautz, D. (2000). Conserved and divergent aspects of terminal patterning in the beetle Tribolium castaneum. Proc Natl Acad Sci U A 97, 6591-6.

Schröder, R., Beermann, A., Wittkopp, N. and Lutz, R. (2008). From development to biodiversity-Tribolium castaneum, an insect model organism for short germband development. Dev. Genes Evol. 218, 119-126.

Schweisguth, F. (2004). Regulation of notch signaling activity. Curr. Biol. CB 14, R129-138.

Schwirz, J. (2014). Systematic Reverse Genetic Screen to Identify Novel Genes Required for Anterior Patterning of the Red Flour Beetle Tribolium castaneum.

Selkoe, D. and Kopan, R. (2003). Notch and Presenilin: regulated intramembrane proteolysis links development and degeneration. Annu. Rev. Neurosci. 26, 565-597.

Seugnet, L., Simpson, P. and Haenlin, M. (1997). Requirement for dynamin during Notch signaling in Drosophila neurogenesis. Dev. Biol. 192, 585-598.

Shapiro, S. S. and Wilk, M. B. (1965). An analysis of variance test for normality (complete samples). Biometrika 52, 591-611.

Simpson, P. (1990). Notch and the choice of cell fate in Drosophila neuroepithelium. Trends Genet. 6, 343-345.

Smith, F. W., Angelini, D. R., Gaudio, M. S. and Jockusch, E. L. (2014). Metamorphic labral axis patterning in the beetle Tribolium castaneum requires multiple upstream, but few downstream, genes in the appendage patterning network. Evol. Dev. 16, 7891.

Snodgrass, R. E. (1935). Principles of Insect Morphology. New York: McGRaw Hill.

Sokoloff, A., Slatis, H. M. and Stanley, J. (1960). The black Mutation in Tribolium castaneum. J. Hered. 52, 131-135.

Speicher, S. A., Thomas, U., Hinz, U. and Knust, E. (1994). The Serrate locus of Drosophila and its role in morphogenesis of the wing imaginal discs: control of cell proliferation. Dev. Camb. Engl. 120, 535-544. 
Srinivasan, S., Armstrong, J. A., Deuring, R., Dahlsveen, I. K., McNeill, H. and Tamkun, J. W. (2005). The Drosophila trithorax group protein Kismet facilitates an early step in transcriptional elongation by RNA Polymerase II. Development 132, 1623-35.

Srinivasan, S., Dorighi, K. M. and Tamkun, J. W. (2008). Drosophila Kismet regulates histone $\mathrm{H} 3$ lysine 27 methylation and early elongation by RNA polymerase II. PLoS Genet 4, e1000217.

Stauber, M., Jäckle, H. and Schmidt-Ott, U. (1999). The anterior determinant bicoid of Drosophila is a derived Hox class 3 gene. Proc. Natl. Acad. Sci. U. S. A. 96, 37863789.

St Johnston, D. and Nüsslein-Volhard, C. (1992). The origin of pattern and polarity in the Drosophila embryo. Cell 68, 201-219.

Strobl, F. and Stelzer, E. H. K. (2014). Non-invasive long-term fluorescence live imaging of Tribolium castaneum embryos. Development 141, 2331-2338.

Struhl, G. and Adachi, A. (1998). Nuclear access and action of notch in vivo. Cell 93, 649660.

Tautz, D. (2004). Segmentation. Dev Cell 7, 301-12.

Tautz, D., Friedrich, M. and Schröder, R. (1994). Insect embryogenesis - what is ancestral and what is derived? Development 1994, 193-199.

Thomas, U., Speicher, S. A. and Knust, E. (1991). The Drosophila gene Serrate encodes an EGF-like transmembrane protein with a complex expression pattern in embryos and wing discs. Dev. Camb. Engl. 111, 749-761.

Toegel, J. P., Wimmer, E. A. and Prpic, N.-M. (2008). Loss of spineless function transforms the Tribolium antenna into a thoracic leg with pretarsal, tibiotarsal, and femoral identity. Dev. Genes Evol. 219, 53-58.

Tomoyasu, Y. and Denell, R. E. (2004). Larval RNAi in Tribolium (Coleoptera) for analyzing adult development. Dev Genes Evol 214, 575-8.

Tomoyasu, Y., Miller, S. C., Tomita, S., Schoppmeier, M., Grossmann, D. and Bucher, G. (2008). Exploring systemic RNA interference in insects: a genome-wide survey for RNAi genes in Tribolium. Genome Biol. 9, R10.

Tornøe, C. W., Christensen, C. and Meldal, M. (2002). Peptidotriazoles on solid phase: [1,2,3]-triazoles by regiospecific copper(i)-catalyzed 1,3-dipolar cycloadditions of terminal alkynes to azides. J. Org. Chem. 67, 3057-3064.

Trauner, J., Schinko, J., Lorenzen, M. D., Shippy, T. D., Wimmer, E. A., Beeman, R. W., Klingler, M., Bucher, G. and Brown, S. J. (2009). Large-scale insertional mutagenesis of a coleopteran stored grain pest, the red flour beetle Tribolium castaneum, identifies embryonic lethal mutations and enhancer traps. BMC Biol 7, 73.

Tribolium Genome Sequencing Consortium, Richards, S., Gibbs, R. A., Weinstock, G. M., Brown, S. J., Denell, R., Beeman, R. W., Gibbs, R., Beeman, R. W., Brown, S. J., et al. (2008). The genome of the model beetle and pest Tribolium castaneum. Nature 452, 949-955.

Turner, F. R. and Mahowald, A. P. (1979). Scanning electron microscopy of Drosophila melanogaster embryogenesis: III. Formation of the head and caudal segments. Dev. Biol. 68, 96-109.

Van der Zee, M., Berns, N. and Roth, S. (2005). Distinct functions of the Tribolium zerknullt genes in serosa specification and dorsal closure. Curr Biol 15, 624-36.

Van der Zee, M., Stockhammer, O., von Levetzow, C., Nunes da Fonseca, R. and Roth, S. (2006). Sog/Chordin is required for ventral-to-dorsal Dpp/BMP transport and head formation in a short germ insect. Proc Natl Acad Sci U A 103, 16307-12.

VanHook, A. and Letsou, A. (2008). Head involution in Drosophila: genetic and morphogenetic connections to dorsal closure. Dev. Dyn. Off. Publ. Am. Assoc. Anat. 237, 28-38. 
Vincent, A., Blankenship, J. T. and Wieschaus, E. (1997). Integration of the head and trunk segmentation systems controls cephalic furrow formation in Drosophila. Development 124, 3747-54.

Wang, W. and Struhl, G. (2004). Drosophila Epsin mediates a select endocytic pathway that DSL ligands must enter to activate Notch. Dev. Camb. Engl. 131, 5367-5380.

Wang, W. and Struhl, G. (2005). Distinct roles for Mind bomb, Neuralized and Epsin in mediating DSL endocytosis and signaling in Drosophila. Dev. Camb. Engl. 132, 2883-2894.

Wang, L., Wang, S., Li, Y., Paradesi, M. S. R. and Brown, S. J. (2007). BeetleBase: the model organism database for Tribolium castaneum. Nucleic Acids Res. 35, D476-479.

Watson, F. L., Püttmann-Holgado, R., Thomas, F., Lamar, D. L., Hughes, M., Kondo, M., Rebel, V. I. and Schmucker, D. (2005). Extensive diversity of Ig-superfamily proteins in the immune system of insects. Science 309, 1874-1878.

Weissman, A. M. (2001). Themes and variations on ubiquitylation. Nat. Rev. Mol. Cell Biol. 2, 169-178.

Wharton, K. A., Johansen, K. M., Xu, T. and Artavanis-Tsakonas, S. (1985). Nucleotide sequence from the neurogenic locus notch implies a gene product that shares homology with proteins containing EGF-like repeats. Cell 43, 567-581.

Wheeler, S. R., Carrico, M. L., Wilson, B. A., Brown, S. J. and Skeath, J. B. (2003). The expression and function of the achaete-scute genes in Tribolium castaneum reveals conservation and variation in neural pattern formation and cell fate specification. Development 130, 4373-81.

Wiellette, E. L. and McGinnis, W. (1999). Hox genes differentially regulate Serrate to generate segment-specific structures. Dev. Camb. Engl. 126, 1985-1995.

Wimmer, E. A., Cohen, S. M., Jackle, H. and Desplan, C. (1997). buttonhead does not contribute to a combinatorial code proposed for Drosophila head development. Development 124, 1509-17.

Winick, J., Abel, T., Leonard, M. W., Michelson, A. M., Chardon-Loriaux, I., Holmgren, R. A., Maniatis, T. and Engel, J. D. (1993). A GATA family transcription factor is expressed along the embryonic dorsoventral axis in Drosophila melanogaster. Development 119, 1055-1065.

Wu, J. and Cohen, S. M. (1999). Proximodistal axis formation in the Drosophila leg: subdivision into proximal and distal domains by Homothorax and Distal-less. Dev. Camb. Engl. 126, 109-117.

Yeh, E., Zhou, L., Rudzik, N. and Boulianne, G. L. (2000). Neuralized functions cell autonomously to regulate Drosophila sense organ development. EMBO J. 19, 48274837.

Younossi-Hartenstein, A., Tepass, U. and Hartenstein, V. (1993). Embryonic origin of the imaginal discs of the head of Drosophila melanogaster. Rouxs Arch. Dev. Biol. 203, 60-73.

Zarin, A. A., Daly, A. C., Hülsmeier, J., Asadzadeh, J. and Labrador, J.-P. (2012). A GATA/homeodomain transcriptional code regulates axon guidance through the Unc-5 receptor. Dev. Camb. Engl. 139, 1798-1805.

Zarin, A. A., Asadzadeh, J., Hokamp, K., McCartney, D., Yang, L., Bashaw, G. J. and Labrador, J.-P. (2014). A transcription factor network coordinates attraction, repulsion, and adhesion combinatorially to control motor axon pathway selection. Neuron 81, 1297-1311. 


\section{Appendix}

\subsection{Primer list}

Table 7.1: Primer list- The name of the gene, the name of the primer and primer sequence are indicated.

\begin{tabular}{|c|c|c|}
\hline Gene & Primer & Sequence \\
\hline \multirow[t]{8}{*}{ TC006255 } & 1021_f_1 & CAAGCGAAAGTGTGCAAAAC \\
\hline & 1021_r_1 & TACTGCCCATACCTTGCATC \\
\hline & 1021_f_2 & GTGTTTGCAAGCAAGCGAAAGTGT \\
\hline & 1021_r_2 & ATAGGAAGGGATGATCGGCT \\
\hline & 1021_f1 & GCAGATGTGTACAAGGAAATC \\
\hline & 1021_r1 & GAAGTAAAGGGCGTTTATGA \\
\hline & 1021_f2 & GCCCAACTGGAACAGGTCTA \\
\hline & 1021_r2 & ACCTCGGGCGAAACAACT \\
\hline \multirow[t]{4}{*}{ TC010758 } & 1725_f1 & ACGAAGTTGTCAGAACGAGTA \\
\hline & 1725_r1 & GACTGGAGCAAGCTTCTG \\
\hline & 1725_f2 & AGTCGTCTGGAATCCTAATTG \\
\hline & 1725_r2 & CAGTGATAGTGATCGTTTTGC \\
\hline \multirow[t]{4}{*}{ TC005877 } & 951_f1 & ACCACAAGGGCCGTAATC \\
\hline & 951_r2 & GACGCCTTTGGCCTTAAC \\
\hline & 951_f2 & CACGGAAGTAGACAGTAAGC \\
\hline & 951_r2 & GAGCTGACTTGAGTTTCG \\
\hline \multirow[t]{4}{*}{ TC003368 } & 561_f1 & GACTGAACTAGTGCTCAGCTCA \\
\hline & 561_r2 & CGAACATGAACGTGTCAATC \\
\hline & $561 \_\mathrm{f} 2$ & GCGGTAATCGACAACATC \\
\hline & $561 \_r 2$ & CATGCCTATGCTGGAGTC \\
\hline \multirow[t]{4}{*}{ TC003442 } & 3557_f1 & CAGGGCATGAACCAGTCTG \\
\hline & 3557_r1 & GTAACCTCGCCTGGTCAATTC \\
\hline & 3557_f2 & GACGAAATACACCCAGAGTTG \\
\hline & 3557_r2 & CGGTCTCCACTAGCCCTATC \\
\hline \multirow[t]{4}{*}{ TC010938 } & 1766_f1 & CGGTTTCAGTCAATGCGA \\
\hline & 1766_r1 & CGTGTAATCGGGGATTTTAATC \\
\hline & 1766_f2 & CTAGCGATGAAACTTCTGGACTA \\
\hline & 1766_r2 & GACCCGTAAGGACCAATTCTA \\
\hline TC012475 & 5247_f1 & CACGAGGAAGAGCCGATA \\
\hline
\end{tabular}




\begin{tabular}{|c|c|c|}
\hline & 5247_r1 & CGTAGCAAACTGGCGTTAC \\
\hline & 5247_f2 & GACGTGATCTTGGTCACTTAC \\
\hline & 5247_r2 & CGCCACAGACATTAGAATG \\
\hline \multirow[t]{2}{*}{ TC016377 } & 02582_cds_F1 & GTAGTTGTGGAAATTCCTGA \\
\hline & 02582_cds_R1 & GCTATGTCTTGGGCAGTA \\
\hline \multirow[t]{2}{*}{ TC012539 } & 05264_cds_F3 & TCGTTTGTGTCCGATCATGT \\
\hline & 05264_cds_R3 & CCATCATTCGTGTGAATTGC \\
\hline \multirow[t]{2}{*}{ TC014275 } & 02268_cds_F3 & CGGAAGAACACGGAGATG \\
\hline & 02268_cds_R3 & CCAGATAATGCCTGACGACT \\
\hline \multirow[t]{2}{*}{ TC014445 } & 05634_cds_F1 & TAGGCACCGTCCGTAACTTC \\
\hline & 05634_cds_R1 & TTTGCTAGCCACTTTGGTGA \\
\hline \multirow[t]{4}{*}{ TC004781 } & 756_f1 & GACGATGATGCGGTTGAGT \\
\hline & 756_r2 & CGGCTCCTGGATGTCTCTGTA \\
\hline & 756_f2 & CGAACAGTTCAGTCGTAATC \\
\hline & 756_r2 & CTGAATTCGTGCTAGATTGA \\
\hline \multirow[t]{4}{*}{ TC014911 } & 2350_f1 & ACGACGATTGCGACGACT \\
\hline & 2350_r1 & GCGATCCCGCCTACTGTA \\
\hline & 2350_f2 & GTACACGAAGCGGTACGA \\
\hline & 2350_r2 & ACTAGCGACTTTACCGTCAGT \\
\hline \multirow[t]{2}{*}{ TC002315 } & nof2_grn_fwd & TGGAGATGACGAACGAACGAGTCT \\
\hline & nof2_grn_rev & ATAGCCAAGGGTGGAGGG \\
\hline
\end{tabular}

\subsection{Non-overlapping fragments synthesized by Eupheria}

Table 7.2 - Primer for non-overlapping fragments synthesized by Eupheria

\begin{tabular}{|l|r|l|l|}
\hline Gene & NOF & Primer left & \multicolumn{2}{l|}{ Primer right } \\
\hline TC006711 & 2 & CGCCCTCTGGAGACAAATAG & GTTTTGATCGCTTCCTCCTG \\
\hline TC014445 & 2 & CAACACGACCTGGACCTACA & TCCAGCAACATCTTCACGAC \\
\hline TC014275 & 2 & CCTCGGCTACTCAAACCAAC & GTTGTTGGGGGCCATATTC \\
\hline TC006291 & 2 & TGACAGTGGGGTGTTTTCAA & CTTCCTCCAAGTCCCATCCT \\
\hline TC012539 & 2 & GTTTTGGGTTACCGCAGCTA & CCACACAGCAACTTCTCCAA \\
\hline TC003063 & 3 & TGTTAGGTCAGCGTTTGCTC & ATGCCAGAGCTGCAGTAGGT \\
\hline & 2 & GGACGGAACGCTGAGTTATG & CTCGTTGTGGAGGTCGATTT \\
\hline TC016377 & 2 & TGGTAATTCCAAGGGGTTTG & CTTGTTTCATGGGCCTGTTT \\
\hline
\end{tabular}

\subsection{Number of proliferating cells in wild type and Tc-mib1 RNAi Embryos}

Table 7.3: Numbers of proliferating cells per embryo - Cells were counted in three different areas on both sides of each embryo (see Figure 4.16A).

\begin{tabular}{l|ll|ll|l|l|} 
Treatment & Head lobe left & Head lobe right & Labrum left & Labrum right & Leg left & Leg right
\end{tabular}




\begin{tabular}{|c|c|c|c|c|c|c|}
\hline WT & 10 & 1 & 98 & 62 & 11 & 24 \\
\hline WT & 12 & 7 & 84 & 80 & 35 & 29 \\
\hline WT & 9 & 12 & 69 & 105 & 124 & 202 \\
\hline WT & 18 & 17 & 58 & 70 & 157 & 131 \\
\hline WT & 29 & 15 & 100 & 92 & 122 & 93 \\
\hline WT & 24 & 2 & 75 & 127 & 135 & 218 \\
\hline WT & 13 & 13 & 101 & 113 & 127 & 164 \\
\hline WT & 20 & 6 & 119 & 106 & 117 & 59 \\
\hline WT & 15 & 12 & 77 & 95 & 75 & 72 \\
\hline WT & 22 & 9 & 105 & 96 & 140 & 112 \\
\hline WT & 11 & 17 & 117 & 93 & 43 & 65 \\
\hline WT & 26 & 29 & 88 & 84 & 178 & 162 \\
\hline WT & 4 & 6 & 84 & 69 & 95 & 36 \\
\hline WT & 27 & 25 & 66 & 57 & 112 & 115 \\
\hline WT & 19 & 42 & 95 & 59 & 140 & 126 \\
\hline WT & 9 & 13 & 78 & 65 & 15 & 55 \\
\hline WT & 17 & 20 & 102 & 132 & 105 & 111 \\
\hline WT & 14 & 8 & 44 & 27 & 40 & 10 \\
\hline WT & 17 & 9 & 81 & 92 & 213 & 128 \\
\hline WT & 27 & 11 & 81 & 52 & 168 & 196 \\
\hline WT & 1 & 3 & 74 & 59 & 45 & 49 \\
\hline WT & 9 & 18 & 41 & 55 & 109 & 111 \\
\hline mib1_RNAi & 23 & 22 & 73 & 83 & 103 & 103 \\
\hline mib1_RNAi & 1 & 5 & 12 & 21 & 63 & 66 \\
\hline mib1_RNAi & 10 & 12 & 25 & 25 & 105 & 77 \\
\hline mib1_RNAi & 27 & 9 & 66 & 54 & 91 & 100 \\
\hline mib1_RNAi & 0 & 0 & 37 & 37 & 111 & 137 \\
\hline mib1_RNAi & 21 & 11 & 47 & 16 & 101 & 89 \\
\hline mib1_RNAi & 32 & 15 & 61 & 43 & 92 & 105 \\
\hline mib1_RNAi & 10 & 13 & 63 & 68 & 132 & 124 \\
\hline mib1_RNAi & 27 & 22 & 90 & 75 & 158 & 125 \\
\hline mib1_RNAi & 12 & 12 & 62 & 64 & 130 & 121 \\
\hline mib1_RNAi & 24 & 17 & 45 & 46 & 146 & 147 \\
\hline mib1_RNAi & 0 & 5 & 70 & 51 & 37 & 45 \\
\hline mib1_RNAi & 21 & 23 & 25 & 64 & 98 & 140 \\
\hline mib1_RNAi & 2 & 0 & 44 & 44 & 203 & 213 \\
\hline mib1_RNAi & 22 & 13 & 85 & 74 & 212 & 182 \\
\hline mib1_RNAi & 18 & 37 & 88 & 82 & 152 & 143 \\
\hline mib1_RNAi & 9 & 6 & 32 & 37 & 80 & 101 \\
\hline mib1_RNAi & 21 & 20 & 71 & 32 & 132 & 118 \\
\hline mib1_RNAi & 5 & 18 & 75 & 84 & 123 & 158 \\
\hline mib1_RNAi & 18 & 32 & 67 & 75 & 87 & 117 \\
\hline mib1_RNAi & 42 & 59 & 72 & 77 & 111 & 105 \\
\hline mib1_RNAi & 1 & 2 & 70 & 40 & 100 & 113 \\
\hline mib1_RNAi & 10 & 3 & 30 & 30 & 137 & 122 \\
\hline mib1_RNAi & 23 & 17 & 61 & 59 & 130 & 126 \\
\hline
\end{tabular}




\begin{tabular}{|c|c|c|c|c|c|c|}
\hline mib1_RNAi & 3 & 0 & 29 & 72 & 127 & 122 \\
\hline mib1_RNAi & 0 & 10 & 46 & 27 & 155 & 162 \\
\hline mib1_RNAi & 4 & 0 & 62 & 37 & 143 & 138 \\
\hline mib1_RNAi & 13 & 11 & 29 & 45 & 99 & 110 \\
\hline mib1_RNAi & 12 & 3 & 77 & 78 & 168 & 153 \\
\hline mib1_RNAi & 3 & 0 & 26 & 44 & 141 & 94 \\
\hline mib1_RNAi & 0 & 0 & 52 & 49 & 144 & 138 \\
\hline mib1_RNAi & 6 & 19 & 56 & 90 & 112 & 103 \\
\hline mib1_RNAi & 2 & 11 & 58 & 71 & 145 & 115 \\
\hline mib1_RNAi & 12 & 8 & 51 & 64 & 68 & 35 \\
\hline mib1_RNAi & 25 & 7 & 55 & 51 & 150 & 138 \\
\hline mib1_RNAi & 0 & 0 & 30 & 31 & 192 & 134 \\
\hline mib1_RNAi & 0 & 3 & 38 & 24 & 199 & 151 \\
\hline mib1_RNAi & 4 & 1 & 9 & 32 & 42 & 37 \\
\hline
\end{tabular}

Table 7.3: Numbers of proliferating cells per embryo - Mean values of proliferating cells for calculation of statistical analyses between wild type and RNAi (see Figure 4.16E).

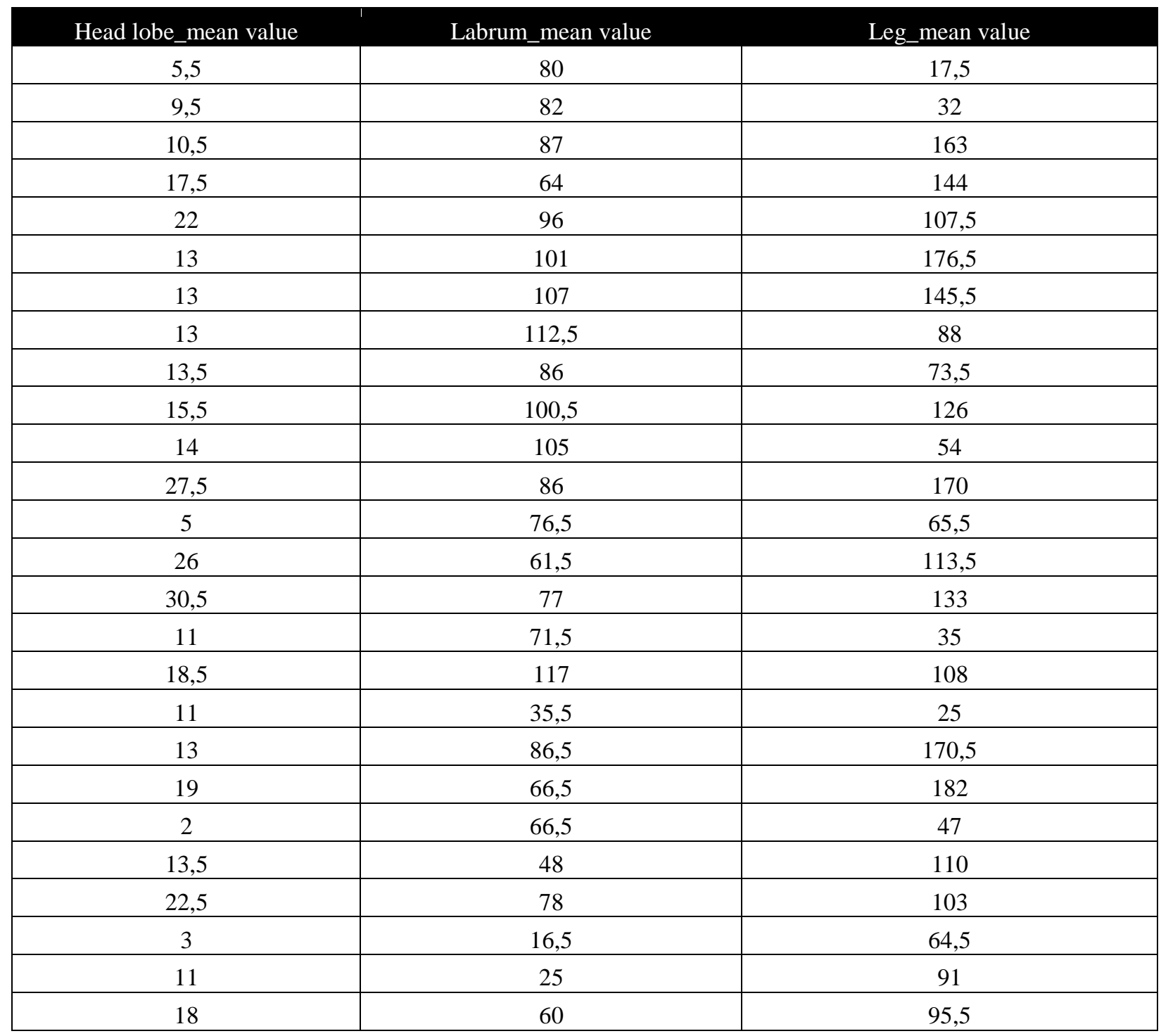




\begin{tabular}{|c|c|c|}
\hline 0 & 37 & 124 \\
\hline 16 & 31,5 & 95 \\
\hline 23,5 & 52 & 98,5 \\
\hline 11,5 & 65,5 & 128 \\
\hline 24,5 & 82,5 & 141,5 \\
\hline 12 & 63 & 125,5 \\
\hline 20,5 & 45,5 & 146,5 \\
\hline 2,5 & 60,5 & 41 \\
\hline 22 & 44,5 & 119 \\
\hline 1 & 44 & 208 \\
\hline 17,5 & 79,5 & 197 \\
\hline 27,5 & 85 & 147,5 \\
\hline 7,5 & 34,5 & 90,5 \\
\hline 20,5 & 51,5 & 125 \\
\hline 11,5 & 79,5 & 140,5 \\
\hline 25 & 71 & 102 \\
\hline 50,5 & 74,5 & 108 \\
\hline 1,5 & 55 & 106,5 \\
\hline 6,5 & 30 & 129,5 \\
\hline 20 & 60 & 128 \\
\hline 1,5 & 50,5 & 124,5 \\
\hline 5 & 36,5 & 158,5 \\
\hline 2 & 49,5 & 140,5 \\
\hline 12 & 37 & 104,5 \\
\hline 7,5 & 77,5 & 160,5 \\
\hline 1,5 & 35 & 117,5 \\
\hline 0 & 50,5 & 141 \\
\hline 12,5 & 73 & 107,5 \\
\hline 6,5 & 64,5 & 130 \\
\hline 10 & 57,5 & 51,5 \\
\hline 16 & 53 & 144 \\
\hline 0 & 30,5 & 163 \\
\hline 1,5 & 31 & 175 \\
\hline 2,5 & 20,5 & 39,5 \\
\hline
\end{tabular}


Figure 7.1: Comparison of proliferating cells between left and right side of each embryo - (A) wild type. (B) Tc-mib1 RNAi. No significant difference in proliferating cells between the left and the right side of each embryo was observed.

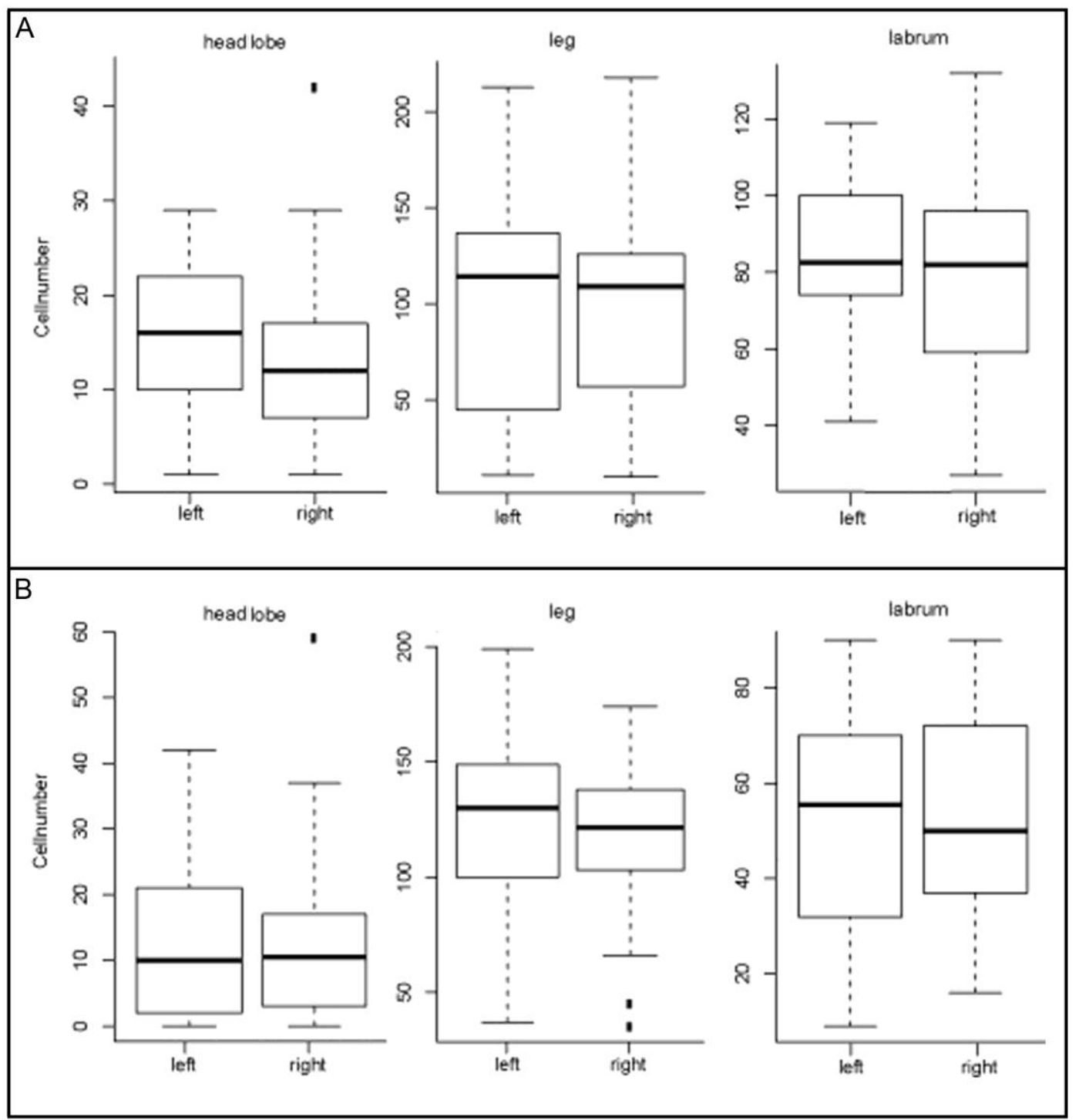




\section{Curriculum vitae}

\section{Address}

Rote Str. 11

37073 Göttingen

Germany

Email: jsieman@gwdg.de

\section{Personal Details}

Name:

Janna Siemanowski

Nationality:

German

Place of birth:

11.01.1986

Date of birth:

47441, Moers

\section{Education}

\section{$\underline{\text { Phd studies }}$}

Since 2011:

Georg-August-University Göttingen

$\mathrm{PhD}$ student under the supervision of Prof. Dr. Gregor Bucher at the Department of Evolutionary Developmental Genetics

Title of thesis: Identification and analysis of novel insect head patterning genes

\section{$\underline{\text { University studies }}$}

2011:

Diploma in Biology of the Ruhr-University-Bochum; Germany 
2010-2011:

Ruhr-University-Bochum, Germany

Diploma thesis under the supervision of Prof. Dr. G. A. Schaub at the Department of Zoology/Parasitology

Title of the thesis: Knockdown und funktionelle Charakterisierung von Cathepsin D in Triatoma infestans (Reduviidae, Insecta)

2008-2010: $\quad$ Main course in Ruhr-University-Bochum; Germany

2005-2008: $\quad$ Basic studies Ruhr-University-Bochum; Germany

\section{High School}

2005 Abitur at Städtische Gesamtschule Kamp-Lintfort, Germany

\section{Language Knowledge}

\section{German}

English 


\section{List of Publications}

Balczun, C., Siemanowski, J., Pausch, J.K., Helling, S., Marcus, K., Stephan, C., Meyer, H.E., Schneider, T., Cizmowski, C., Oldenburg, M., et al. (2012). Intestinal aspartate proteases TiCatD and TiCatD2 of the haematophagous bug Triatoma infestans (Reduviidae): sequence characterisation, expression pattern and characterisation of proteolytic activity. Insect Biochem. Mol. Biol. 42, 240-250.

Oberhofer, G., Grossmann, D., Siemanowski, J. L., Beissbarth, T. and Bucher, G. (2014). Wnt/B-catenin signaling integrates patterning and metabolism of the insect growth zone. Development dev.112797.

Schmitt-Engel, C., Schultheis, D., Schwirz, J., Ströhlein, N., Troelenberg, N., Majumdar, U., Dao, V. A., Grossmann, D., Richter,T.,Tech, M., Dönitz, J., Gerischer,L.,Theis, M., Schild, I., Trauner, J., Koniszewski, N. D. B., Küster, E., Kittelmann, S.,Hu, Y., Lehmann, S., Siemanowski, J.,Ulrich, U., Panfilio, K.A., Schröder, R., Morgenstern, B., Stanke, M., Buchhholz, F., Frasch, M., Roth, S., Wimmer, E.A., Schoppmeier, M., Klingler, M., Bucher G. (accpeted for publication). The iBeetle large scale RNAi screen reveals novel gene functions for insect development and physiology.

Oberhofer, G., Lbik, D., Janna L. Siemanowski, J. L., Bucher, G. (submitted) Fast and robust two-color in situ hybridization in Tribolium castaneum using different combinations of detection systems 